\title{
Review Article \\ Kirkpatrick-Baez (KB) and Lobster Eye (LE) Optics for Astronomical and Laboratory Applications
}

\author{
René Hudec ${ }^{1,2}$ \\ ${ }^{1}$ Astronomical Institute, Academy of Sciences of the Czech Republic, CZ-251 65 Ondrejov, Czech Republic \\ ${ }^{2}$ Faculty of Electrical Engineering, Czech Technical University in Prague, Technicka 2, 16000 Praha 6, Czech Republic
}

Correspondence should be addressed to René Hudec, rene.hudec@gmail.com

Received 16 May 2010; Revised 26 November 2010; Accepted 22 December 2010

Academic Editor: Stephen L. O’Dell

Copyright ( 2010 René Hudec. This is an open access article distributed under the Creative Commons Attribution License, which permits unrestricted use, distribution, and reproduction in any medium, provided the original work is properly cited.

\begin{abstract}
Most of grazing incidence (reflective) X-ray imaging systems used in astronomy and in other (laboratory) applications are based on the Wolter 1 (or modified) arrangement. But there were proposed also other designs and configurations, which are considered for future applications for both in laboratory and (finitely) in space. The Kirkpatrick-Baez (KB) lenses as well as various types of Lobster-Eye (LE) optics and MCP/Micropore optics serve as an example. Analogously to Wolter lenses, the X-rays are mostly reflected twice in these systems to create focal images. The KB systems have already found wide usage in laboratory and synchrotron, both application are reviewed and discussed in detail in this paper. While this paper focuses on future possible applications of non-Wolter grazing incidence systems in space and astronomy, we also discuss in detail applications in other areas of science, where (in contrary to astronomy) some of these systems have demonstrated their advantages.
\end{abstract}

\section{Introduction}

X-ray astronomy represents an important part of recent astrophysics. Many scientific achievements of the last two decades of X-ray astronomy are closely related to the use of imaging X-ray telescopes. These telescopes achieve much better signal/noise ratio than $\mathrm{X}$-ray experiments without optics - this allows, for example, the detection of faint sources. The use of X-ray optics further allows imaging, precise localization, photometry, spectroscopy, variability studies, and estimation of physical parameters of X-ray emitting regions (temperature, electron density, etc.). The space experiments with X-ray optics are also well suited for monitoring of X-ray sky for variable and transient objects including X-ray novae, $\mathrm{X}$-ray transients, $\mathrm{X}$-ray flares on stars and Active Galactic Nuclei (AGNs), galactic bulge sources, X-ray binaries, SGRs (Soft Gamma Ray Repeaters), and X-ray afterglows of GRBs (Gamma Ray Bursts). The Xray optics represents an important part of numerous past, recent, and future space projects such as the European Xray Observatory Satellite (EXOSAT), the Roentgen Satellite (ROSAT), Einstein, RT-4M Salyut 7, Fobos, the Advanced X-ray Astrophysics Facility AXAF/Chandra, the X-ray Multi- mirror Mission (XMM)/Newton, BeppoSAX, the Advanced Satellite for Cosmology and Astrophysics (ASCA), and the International X-Ray Observatory IXO.

In the laboratory, there are numerous applications of the $\mathrm{X}$-ray optics for example, in plasma physics, laser plasma, synchrotron analyses, biology, crystallography, medicine, material and structure testing, X-ray lithography, and so forth. These applications will be reviewed in next sections of this paper, when the various application areas of the nonWolter X-ray optics will be described and discussed.

The alternative (to Wolter systems) X-ray imaging mirrors based on grazing incidence reflections were described in the literature and have found numerous applications in the laboratory, but their space and astronomy applications are still marginal. We review and discuss these systems, discuss their past and recent ground-based applications, and discuss their possible potential for future X-ray astronomy applications.

We give a brief introduction to the various types of grazing incidence X-ray optics in Section 2, with emphasis on the non-Wolter optical systems. In Section 3, the KirkpatrickBaez systems (consisting a set of two orthogonal parabolas of translation) are described and discussed in detail, for 
both laboratory and space and astrophysical applications (yet limited but with promising potential for the future). In Section 4 the wide-field systems of Lobster Eye (LE) are in detail described and discussed, with emphasis on prototypes already designed, developed and tested. Both Schmidt and Angel optics are discussed, as well as MultiFoil (MFO) and MicroChannel Plate (MCP) approaches, including short comparison and recent published results demonstrating the measured performance. In Section 5 capillaries are introduced and described.

1.1. Why X-Ray Optics? There are numerous applications in space and in the laboratory where the $\mathrm{X}$-rays imaging in is required. Very essential is the use of X-ray optics in X-ray astronomy and astrophysics since it improved the signal to noise ratio, and hence much fainter and more distant objects can be detected and investigated.

The grazing incidence reflecting $\mathrm{X}$-ray lenses discussed in this work reflect typically soft X-rays from the optical region up to about $2-10 \mathrm{keV}$, depending on the surface material and on the angle of incidence. Since there were scientific requirements to enlarge the working range toward the higher energies, the recent efforts focus on various improvements and additional surface layers such as multilayer's to meet this $[13,14])$.

In astronomy and astrophysics, it was the use of imaging $\mathrm{X}$-ray telescopes based on grazing incidence X-ray optics that opened a completely new window to the Universe and has lead to great discoveries during the past decades. To acknowledge these achievements, the Nobel Prize for physics for the year 2002 was assigned to the Professor Riccardo Giacconi who significantly contributed to the construction of first astronomical X-ray telescopes in the 1960s and 1970s.

In this work, we focus on the non-Wolter grazing incidence X-ray imaging systems.

1.2. The History of X-Ray Imaging. The X-rays were discovered in 1895 by W. C. Roentgen. However, it took many years to establish the nature of X-rays as electromagnetic waves, and to find ways how to focus them. Essential was the discovery that the X-rays are diffracted when passing through a crystal (von Laue 1912 [16]). The second step for focusing of X-rays was the finding that the $\mathrm{X}$-rays can be reflected from a polished surface at small (glancing, grazing incidence) angles (Compton 1923 [17] and Ehrenberg and Jentzsch 1929 [18]).

It is obvious that classical lenses and others optical telescope designs cannot be used for X-ray focusing and/or imaging, as X-rays are either absorbed or passed straight through lens or mirrors. In addition, any single mirror Xray grazing incidence system suffers from severe astigmatism (Jentzsch 1929 [19]). The first design of X-ray optics was suggested by Kirkpatrick and Baez in 1948. The problem of astigmatism was overcome with the use of 2 orthogonally crossed mirrors, each providing $1 \mathrm{D}$ focusing. This led to the production of the first $2 \mathrm{D} X$-ray image. In the same year, the experiment carried out by a V2 rocket discovered the Sun being a bright source of X-rays (Burnight 1949 [20]). The further important step came in 1951, when German physicist Hans Wolter (Wolter 1952 [1]) published his (different from Kirkpatrick and Baez) idea of grazing incidence Xray mirrors. According to Wolter, a true X-ray image can be formed by two grazing reflections on three various parabolic/hyperbolic or parabolic/elliptic combinations of coaxial and confocal mirrors. The Wolter design is now the most widely one used in X-ray telescopes (e.g., EXOSAT, ROSAT, Chandra, Newton, IXO, etc.).

As already mentioned, the first celestial object seen in Xrays was Sun. However, the first X-ray image of the Sun was not taken by a mirror, but with the use of a simple pinhole camera (Chubb et al. 1961 [21], Byram et al. 1961 [22]). In 1962 , a counter experiment on a sounding rocket discovered the first nonsolar celestial X-ray sources, namely, the diffuse $\mathrm{X}$-ray background and the galactic source Scorpius X-1. In 1963, the first grazing incidence telescope of the Wolter type was used to record the X-ray images of the Sun on a photographic film (in the East, it was a similar experiment in 1978, also using sounding rocket, e.g., [23]). More advanced X-ray mirrors having sub-arcmin resolution came with the US space station Skylab in 1973. The station Skylab carried two solar X-ray telescopes S-054 (Underwood et al. 1977 [24]) and S-056 (Underwood and Attwood 1984 [25], Thiel et al. 1989 [26]) with fine angular resolution of a few arc seconds. The images were recorded on the X-ray film, taking the advantage of telescope being onboard of the manned space vehicle.

After Skylab telescopes, the interest moved towards the non-solar X-ray astronomy and astrophysics (e.g., [27]). The application of focusing X-ray optics to X-ray astronomy was first discussed by Giacconi and Rossi [28]. The most commonly used system since then consists of two successive conic sections of revolution (Magnus and Underwood, 1969 [29]).

The US Einstein observatory (HEAO-B) was launched in 1978, with a large nested (4 shells) X-ray imaging telescope of a Wolter type I configuration. The detectors of this telescope included the imaging proportional counter and a high-resolution microchannel plate device. The European EXOSAT satellite followed in 1983 with two identical meanaperture Wolter-type I telescopes was produced by unique gold/epoxy replication. Also, the Soviet orbital station Salyut 7 carried similar imaging X-ray telescope RT-4M onboard, with an epoxy replicated X-ray mirrors produced by our group in the Czech Republic (Hudec et al., 1988 [30]). The aperture $(24 \mathrm{~cm})$ was similar to those of EXOSAT and the reflecting material was gold for the outer shell and nickel for the inner shell. The progress continued with German satellite ROSAT and recently with Chandra-AXAF and XMM-Newton (with unprecedented collecting area of $\mathrm{XMM}$ - the combined collecting area is $4,300 \mathrm{~cm}^{2}$ - and angular resolution of AXAF $-0.5 \mathrm{arcsec}$ ). Nowadays, future large and advanced X-ray telescopes such as the IXO of the European Space Agency ESA, American agency NASA, and Japanese agency JAXA, or Astro-H by JAXA, are planned and considered. There are numerous and various efforts for these future technologies (e.g., Citterio et al. 2002 [31], Hudec et al. 2004 [32] and Hudec et al. 2006 [33]). 
Apart from these Wolter type imaging systems, other types (non-Wolter) imaging elements based on grazing incidence were proposed and discussed. The KirkpatrickBaez mirror systems were flown several times on rockets; the Lobster Eye modules (e.g., Hudec et al., 2003 [34]) are still waiting for their space applications.

In parallel to space/astronomy oriented efforts, the Xray optics has been developed for various laboratory and technology applications (e.g., sobelmen et al. 1990 [35]). Many of these efforts originated from astronomical X-ray optics developments and related techniques. More recently, as we will show in this paper, the future astronomical instrumentation can benefit from past developments of non-Wolter X-ray optics for laboratory and other nonastronomical applications. Recently available novel X-ray optics substrates such as improved silicon wafers can be favourably used in these systems, both laboratory and astronomical.

In the Czech Republic, the efforts on design and developments of X-ray lenses and X-ray telescopes started in late 60 ies as a part of the Interkosmos program and focused on grazing incidence optics of various types (e.g., Hudec et al. 1981 [36], Hudec et al. 1984 [37], and Sobelman et al. 1990 [38]). Three main designs were investigated and supported, namely Wolter, Kirkpatrick-Baez (KB), and Lobster (Hudec et al. 1989 [39], Hudec et al. 1989 [40], Hudec et al. 1991 [41], Hudec et al. 1992 [42], Hudec et al. 1999 [43], Hudec et al. 2000 [44], Hudec et al. 2001 [45], Hudec et al. 2003 [46], Hudec et al. 2004 [32] and Hudec et al. 2004 [47]).

\section{The Various Types of X-Ray Optics}

According to the physical processes involved in the imaging process, one can divide the X-ray optics into the following groups:

(i) diffractive optics (Fresnel lenses + pinholes): not useful for space due to small apertures (albeit there are recent considerations of applications of analogues of refractive lenses based on mosaic crystals (Laue lenses) in space gamma-ray astronomy),

(ii) refractive optics: limited use, not useful for space,

(iii) reflective optics, based on total reflection (grazing incidence),

(iv) reflective optics, allowing normal incidence (multilayer's).

In this work, we will concentrate on reflective X-ray lenses (mirrors). The critical angle for total external reflection of X-rays from materials composed of heavy elements (Henke et al. 1993 [48], and Hubbell et al. 1975 [49], Hubbell et al. 1977 [49]) can be calculated as (Table 1)

$$
\theta_{c}=2.32 \times 10^{3}\left(\frac{Z_{\rho}}{A}\right)^{1 / 2} \lambda
$$

TABLe 1: Critical angles $\theta_{c}$ (mrad) for selected materials frequently used to reflect X-rays in grazing incidence X-ray mirrors for two selected energies $\mathrm{Cu} K_{\alpha}(8,0 \mathrm{keV})$ and $\mathrm{Mo} K_{\alpha}(17.4 \mathrm{keV})$.

\begin{tabular}{lcc}
\hline Material & $\mathrm{Cu} K_{\alpha}$ & Mo $K_{\alpha}$ \\
\hline $\mathrm{Ni}$ & 7.37 & 3.40 \\
$\mathrm{Au}$ & 9.96 & 4.59 \\
$\mathrm{Ir}$ & 10.75 & 4.96 \\
Glass & 4.07 & 1.88 \\
\hline
\end{tabular}

where $Z, A$ and $\rho$ are the atomic number, atomic mass and specific gravity, respectively, of the surface and $\lambda$ is the $X$-ray wavelength in $\mathrm{nm}$.

This gives a critical angle of about $1^{\circ}$ for X-rays of a few $\mathrm{keV}$. For larger incidence angles, the mirrors will absorb the $\mathrm{X}$-rays. As already mentioned, the $\mathrm{X}$-rays are reflected when they incident on very smooth surfaces at glancing angles less than the critical angle for total external reflection. However the decrease in reflectivity is not sharp because of inherent photoelectric absorption in the material as well as surface roughness of the surface.

Table 1 lists the critical angles for various materials for $\mathrm{Cu} K_{\alpha}$ and Mo $K_{\alpha}$ radiation. Note that for example, the critical angles for Mo $K_{\alpha}$ are so small that it is very difficult to construct reflecting collimators for Mo $K_{\alpha} \mathrm{X}$-rays.

The critical angle, however, is in fact not sharply defined: the reflectivity $R$ drops off slowly from near-unity at a very small glancing angle and the falloff is less sharp for heavy elements than for light ones. The decrease in the reflectivity is a function of the surface micro roughness; for the typical $\mathrm{X}$-ray imaging purposes, the rms roughness must be less than $1 \ldots 2 \mathrm{~nm}$. Values of $R$ as a function of $\theta, \theta_{c}$ and $\sigma$ have been computed using models of differing complexity (see Sinha et al., 1988 [38]; Kopecky, 1995 [50]). The effects can be computed by the Fresnel approximation (see Compton, 1935 [51]) and multiplied by a roughness factor $\gamma_{\sigma}=$ $\exp -\left[(4 \pi \sigma \theta / \lambda)^{2}\right]$. The requirement for grazing incidence reflections leads to telescope designs that are different from classical optical devices.

2.1. Wolter Optics. As seen in the previous sections, the $\mathrm{X}$-rays can be reflected only on smooth surfaces at very small (typically less than 1 degree depending on surface material and the energy range) angles, that is, at grazing incidence. The suitable materials for the mirrors are the metals with high density, such as gold, nickel, and platinum. The reflection is nondispersive, that is, focusing of X-rays is over a broad energy range. The most common type of the grazing incidence optics is the Wolter optics (Wolter, 1952 [1]). Wolter designed an aplanatic system of X-ray grazing incidence mirrors free of both spherical aberration and coma, with the Abbe sine condition fulfilled (Figure 1).

As the Wolter optics is in detail described in other papers in this volume, as well as in many other papers (e.g., Aschenbach 1985 [52]) it will not be described and discussed in this paper. The Wolter optics constructed in the Czech Republic is shown on Figure 2. 

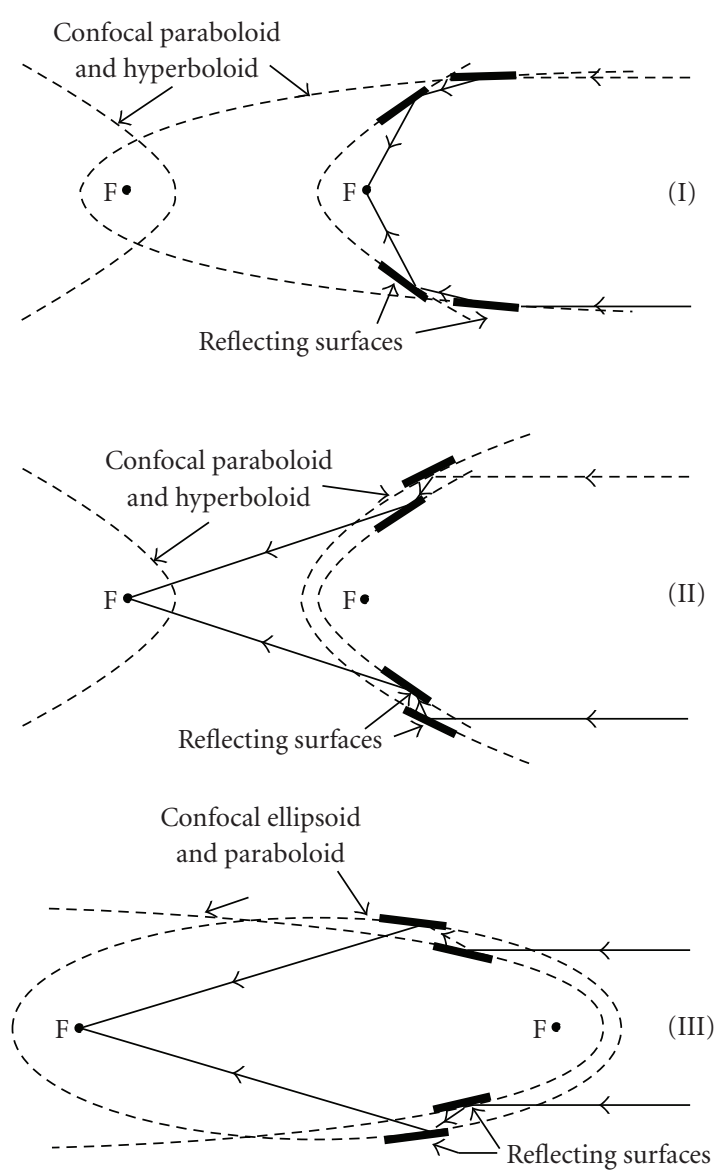

Figure 1: The schematics of Wolter X-ray lenses (according to Wolter 1952 [1]).

2.2. Double-Cone Approximation of Wolter Optics. For some applications, mostly those not requiring high angular resolution, the Wolter surfaces can be approximated by less laborious and hence less expensive conical profiles. The conical mirrors have lower imaging quality, but in many cases larger collecting area since the shells can be made very thin (Serlemitsos et al. 1997 [53]). These mirrors usually represent high-throughput systems, and can be preferably used in astronomical foil telescopes. The foil mirrors use thin foils instead of (thicker) grazing incidence reflecting surfaces. Examples of X-ray telescopes based on this approach are The Broad-Band X-Ray Telescope (BBXRT), Suzaku, and ASCA. In the case of ASCA, each of its 4 telescopes consists of 120 layers, while the Suzaku has about 180 shells in each of the 5 telescopes. The drawback of these and similar foil telescopes based on double conical arrangement is the spatial resolution limited to (in best cases) about $1 \mathrm{arcmin}$. This is also the case of the recent Astro-H telescope. More recently, multipore silicon wafer optics has been proposed (Bavdaz et al., 2004 [54]). It is based on conical approximation but with very special arrangement resulting in very short lengths of the surfaces and hence very small deviations from parabolic and hyperbolic surfaces. As a consequence, such systems could achieve fine angular resolution, of order of 10 arcsec.

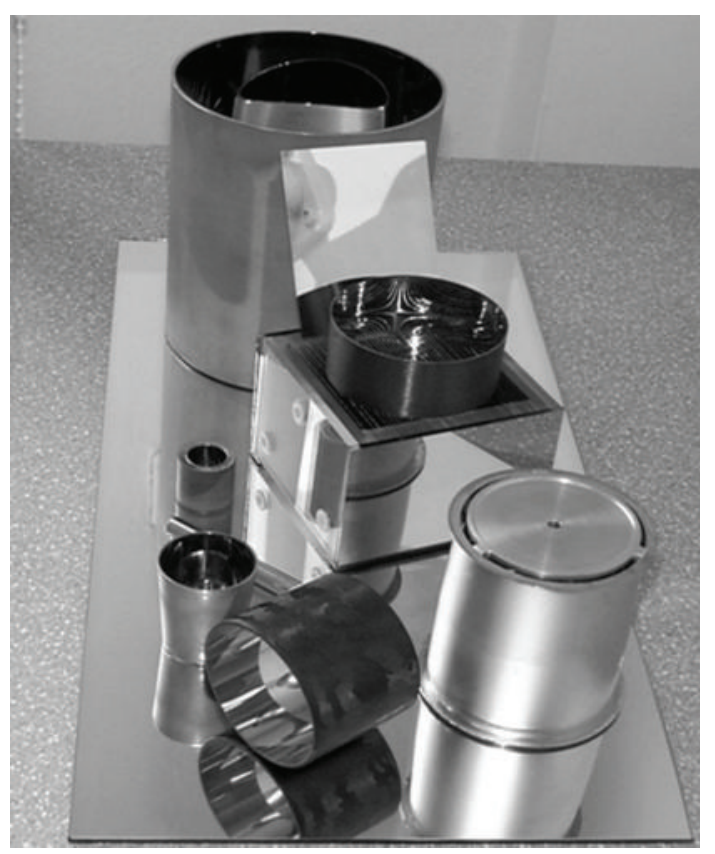

Figure 2: Replicated grazing incidence X-ray optics (various technologies) designed and developed by our group in the Czech Republic. The replication technology has been modified several times to meet the dedicated requirements. The two black mirrors are produced by carbon fibre technology.

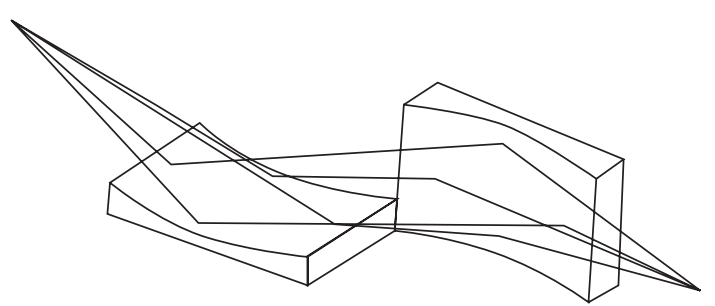

FIGURE 3: The arrangement of the Kirkpatrick-Baez X-ray objective (according to Kirkpatrick and Baez 1948 [2]).

2.3. Conical, Ellipsoidal, and Paraboloidal Optics. These mirrors with only one reflection find most of their applications in the laboratory either as collimating or focusing and/or imaging elements.

2.4. Kirkpatrick-Baez (KB) Optics. Although the Wolter systems are generally well known, Hans Wolter was not the first who proposed X-ray imaging systems based on reflection of $\mathrm{X}$-rays. In fact, the first grazing incidence system to form a real image was proposed by Kirkpatrick and Baez 1948 [2]. This system consists of a set of two orthogonal parabolas of translation as shown in Figure 3. The first reflection focuses to a line, which is focused by the second surface to a point. This was necessary to avoid the extreme astigmatism suffered from a single mirror but still was not free of geometric aberrations. Nevertheless, the system is attractive for being easy to construct the reflecting surfaces. These surfaces can be produced as flat plates and then mechanically 


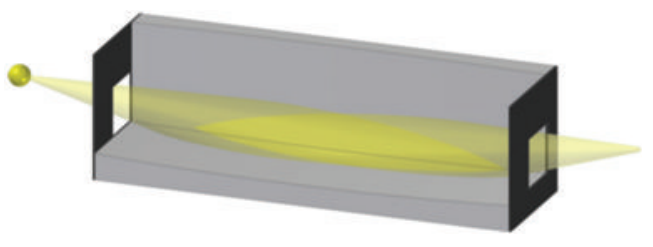

(a)

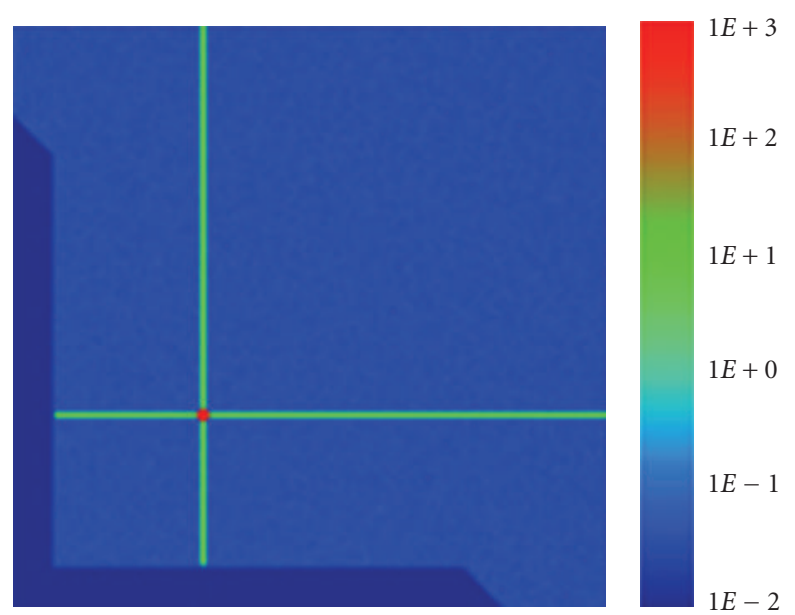

(b)

FIGURE 4: Principle of Montel optics (a) and simulated intensity distribution in the focal plane when no apertures are used (b): shadow of the mirrors (dark blue), direct rays (light blue), line foci (green) and focus point (red). Adopted from http://www.X-ray-optics.de/ [3].

bent to the required curvature. In order to increase the aperture a number of mirrors can be nested together, but it should be noted that such as nesting introduces additional aberrations.

This configuration is used mostly in experiments not requiring large collecting area (solar, laboratory). Recently, however, large modules of KB mirrors have been suggested also for stellar X-ray experiments.

2.5. Montel Optics. Montel optics (Montel 1957 [58]), also known as "side-by-side" optics, consist of two mirrors arranged perpendicular and side-by-side (Figure 4). The mirrors are shaped like elliptic cylinders. In the case of a very distant source or focal point, the incoming, respectively, out coming rays are nearly parallel to the optical axis leading to parabolic shaped cylinders as mirrors. Incoming rays hitting one of the mirrors first and then the other one will be redirected to the focus point. Some rays hit only one of the mirrors resulting in a horizontal or vertical line focus (Figure 4(b)). These rays, and the rays hitting none of the mirrors have to be blocked by suitable shaped apertures at the entrance and at the exit of the optics. This optics has laboratory applications only.

2.6. Normal Incidence Optics. There is one exception for the grazing incidence rule for reflecting X-rays mentioned before, and that is the multilayer normal incidence optics. This type of X-ray optics requires the multilayer deposition on the mirror surface allowing its use under normal incidence (e.g., Alford et al. 1995 [59]). The use of multilayer's results in narrow spectral range and in the past has been used mostly in solar experiments (TEREK Fobos, TEREK KORONAS...) as well as in laboratory applications (Schwarzschild microscopes, etc.). The normal incidence systems are limited to soft X-rays because of the limitation of multilayer's.
2.7. Lobster Eye (LE) Optics. This optic mimics the arrangements of eyes of lobsters and was suggested in the 1970s for a very wide field X-ray imaging but not yet used in space mostly due to severe manufacturing problems. Recently, the first test modules have been available for both the Schmidt and the alternative Angel configurations.

Images in the eye of a lobster are formed through reflections off the internal walls of a lattice of small squaresided tubes arranged over the surface of a sphere. This design can be used in the construction of a grazing incidence system to focus X-rays (Figure 5).

The X-ray objective based on the lobster eye was proposed by Angel (1979 [60]), following a similar design of a wide-field lens by Schmidt (1975 [53]). Each small channel is aligned along the radius of a sphere. A ray reflected twice off adjacent walls inside the channel is focused onto a spherical focal plane. Rays reflected only once are focused to a line causing background images to appear as a tapered cross. Some rays go through the lens with no reflections, contributing to diffuse background. The finite size of the tubes produces specific defocusing in the image, while the angle subtended by each tube at the focus limits the resolution of the system.

Despite these drawbacks, the great advantage of this design is an almost unlimited field of view (Gorenstein 1987 [61]). This makes it ideal for use as an all-sky X-ray monitor (Priedhorsky et al. 1996 [62], Priedhorsky et al. 2000 [62], Fraser 2002 [63], and Fraser 2003 [64],). Up to date, no X-ray telescopes have been launched using lobstereye optics, mainly due to difficulty in manufacturing of the reflective tubes. However, improvements in multichannel plate technology has led to a proposal for a lobster-eye Xray telescope could be placed on the International Space Station (Fraser et al. 2002 [65]) and to an application on BeppiColombo (Fraser et al., 2010 [66]), and the alternative glass foil technology developed in the Czech Republic has lead to the construction of several first telescopes 

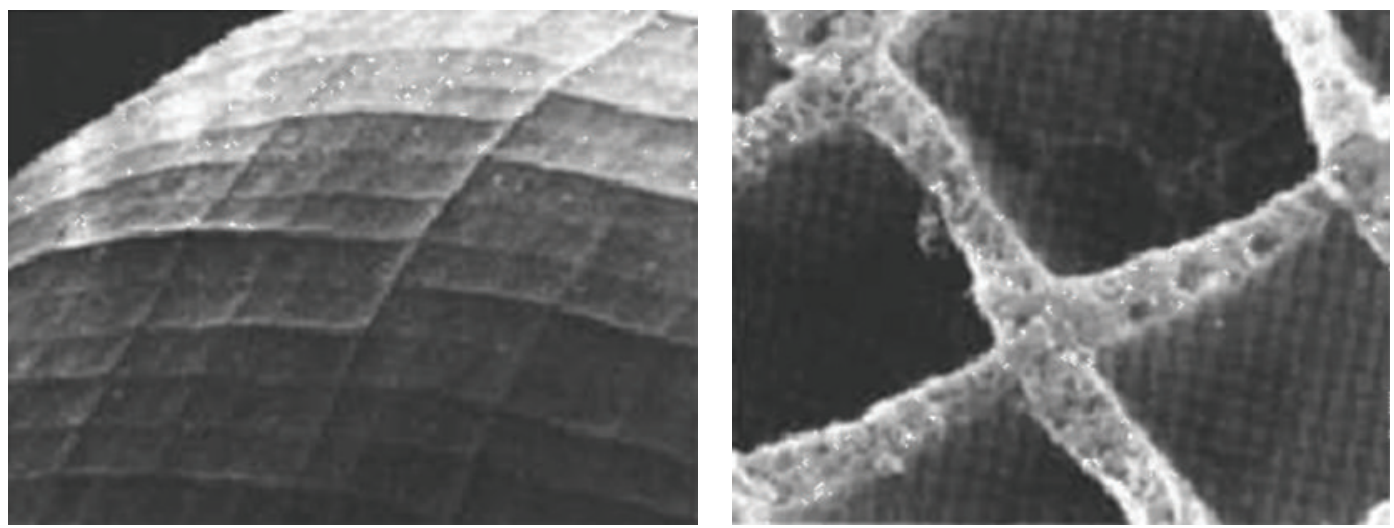

(a)

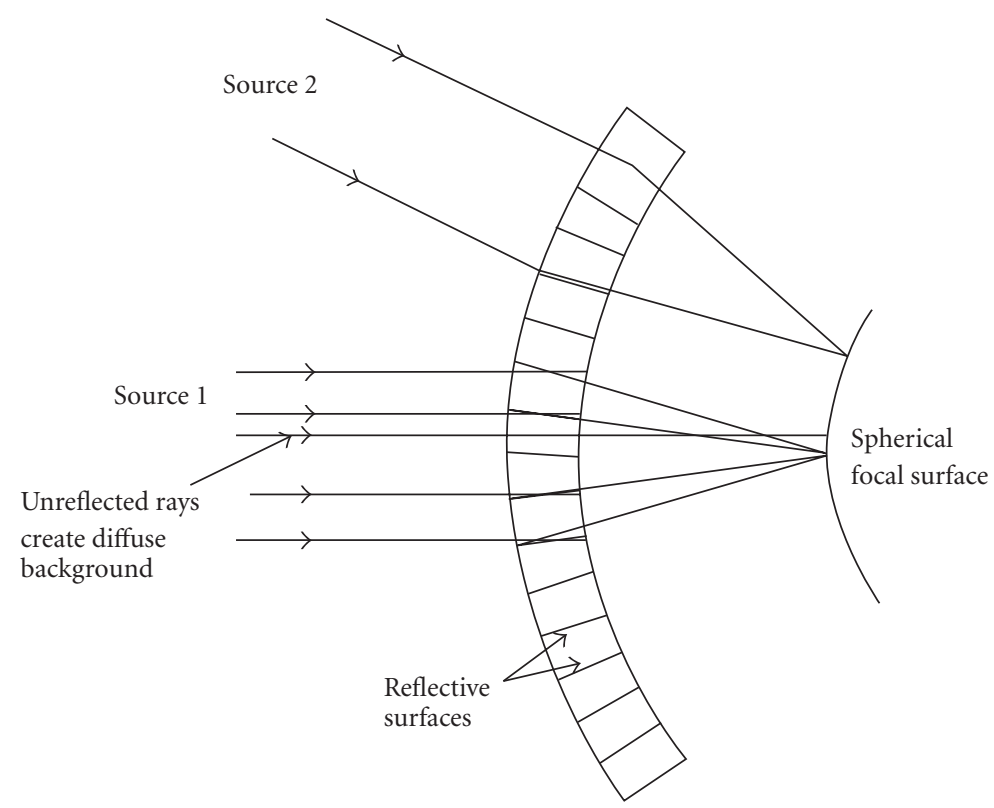

(b)

FIGURE 5: The Lobster-eye wide field X-ray imaging system (from http://www.src.le.ac.uk/lobster). (a) The real lobster eye, (b) the schema.

(Inneman et al. 1999 [67], Inneman et al. 2000 [68]). The related efforts in the Czech Republic have started soon after announcing the idea of lobster optics (Hudec et al., 1985 [69], Hudec et al., 2003 [70]).

2.8. Kumakhov (Polycapillary) X-Ray Optics. Another and fully different type of X-ray optics is based on the fact that the total external reflection also allows X-rays to be transmitted through single straight or tapered hollow glass tubes in analogy to visible light fibre optics (Thiel et al. 1989 [26]). This type of X-ray optic, based on an assembly of a large number of hollow capillary tubes stacked together was invented by Kumakhov (1990 [4]). In this type of X-ray lenses, the Xrays incident on the interior of the tubes at small angles (smaller than the critical angle for total external reflection) is guided down the tubes by total external reflection (Figure 6). Since the original proposal the technology to control X- rays using systematic arrangements of glass polycapillaries has developed significantly and depending on the specific arrangement and application, X-ray beams can be focused or divergent beams can be made quasiparallel.

Such arrays can control X-ray beams, such as collecting divergent radiation from a point source, collimating and focusing (Kumakhov, 1990 [4]). These X-ray optical systems can collect divergent radiation from a point source over a solid angle as large as one radian. Recently, capillary optics can be operated from 1 to $60 \mathrm{keV}$ however, this range is expected to be further extended. These systems find applications in the laboratory but hardly in space telescopes because they do not represent true imaging devices.

2.9. MCP X-Ray Optics. The MicroChannel Plates (MCPs) also focus X-rays by reflection from the inside surfaces of the channels. This is easy to visualise in the simplest possible 

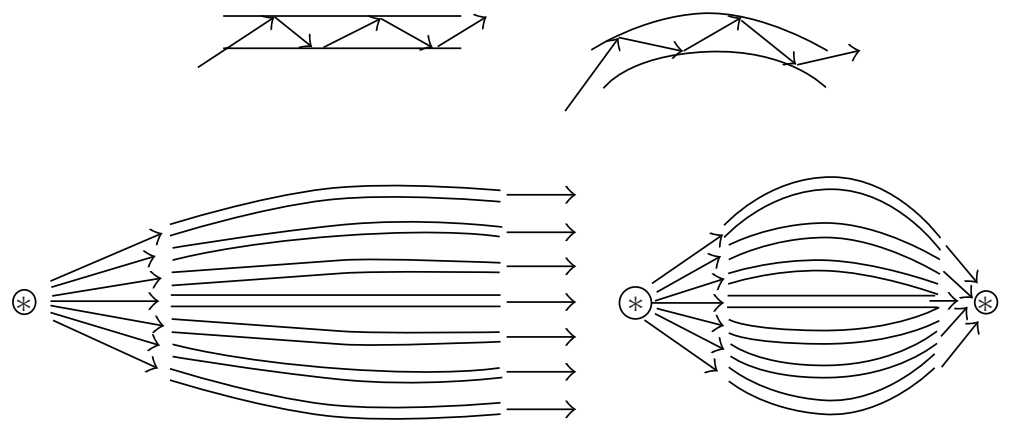

Figure 6: The Kumakhov X-ray lenses (polycapillary optics, Kumakhov, 1990 [4]) left arrangement for a quasiparallel beam, right arrangement for-point-to point focussing.

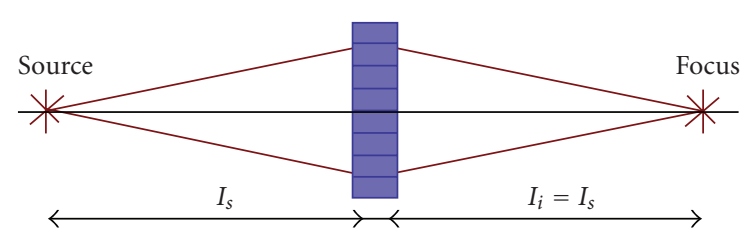

Figure 7: The principle of MCP X-ray Optics.

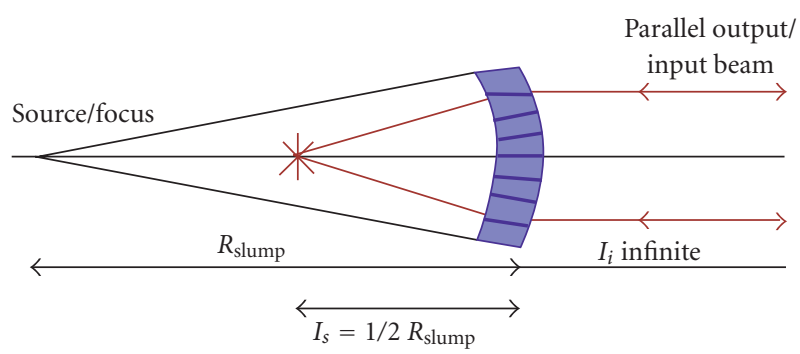

FIgURE 8: The MCP optics as Wolter lens.

case of "point-to-point" focusing using an MCP with square cross-section channels (Figure 7). Recently, a modified MCP $\mathrm{X}$-ray lens technology is called also X-ray MultiPore Optics.

The image is formed at the same distance from the plate as the source and the picture can be extended into two dimensions to allow focusing of a point source by an array of square channels such as an MCP, with the rays reflected twice. The MCP, however, focuses not every ray perfectly-a ray can go straight through a channel without reflection, so ending up either above or below the focus, causing a diffuse halo around the image, like in the lobster eye X-ray lens. Moreover, a ray can be reflected only once, so this ray will be focused in one-dimension only and consequently ending up somewhere along a line through the focus. This causes a cross-like image centred on the focus. About one quarter of the rays go into the "true" or point focus, one quarter into each line focus and one quarter into the diffuse halo.

An important modification occurs if the MCP is slumped to a spherical shape of radius $R_{\text {slump }}$, then it can be used to focus parallel rays to a point and/or to generate a parallel beam from a point source (Figure 8).

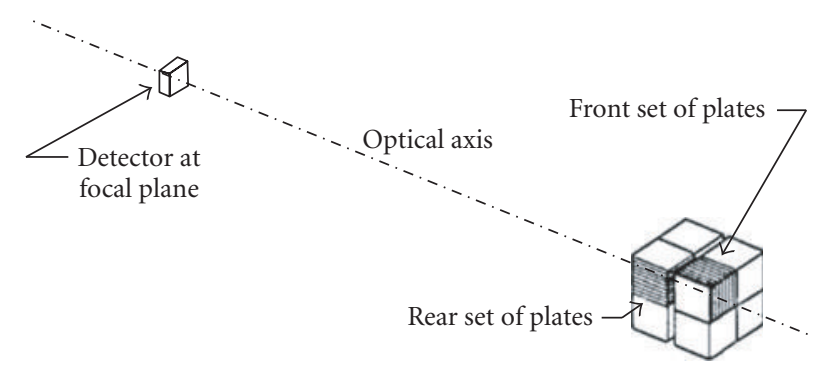

Figure 9: The KB optics design studied for HEAO-A (Kast 1975 [5]).

Two MCP-based designs have been suggested and exploited so far for the X-ray optics: the micro channel wolter optics and the lobster optics (Peele et al. 1996 [76]). In the first one, two MCPs are used as X-ray lenses, representing a conical approximation of a Wolter lens (Wallace et al. 1985 [77]).

\section{Kirkpatrick-Baez X-Ray Optics}

3.1. Kirkpatrick-Baez (KB) Telescopes. Two-dimensional Xray image with grazing incidence reflection was taken by a Kirkpatrick-Baez system (Kirkpatrick and Baez 1948 [2]). It was the first two-dimensional X-ray image ever obtained in the laboratory. The configuration is shown in Figure 4. The incident rays are focused to a line image by a parabolic sheet mirror. If the rays are reflected a second time from a parabolic surface oriented at right angle to the first one, a point-like focus is achieved. This is correct for rays parallel to the centre line of the parabolas. In order to increase the collecting area, a stack of parabolas of translations constructed (Figure 9). Whereas in the case of only one double plate system a perfect focus for on-axis rays can be achieved, this is not possible for a multiple plate arrangement, where the focus remains perfect only along the projected direction of the surface normal of the primary. The exact solution for the intersection point with the focal plane of an arbitrary incident ray is given in the paper by Van Speybroeck et al. (1971 [78]). A detailed configurational analysis of the multiplate Kirkpatrick-Baez system has been carried out by Kast (1975 [5]). 
As already mentioned, the Kirkpatrick-Baez (KB) Xray optics was actually the first X-ray optics suggested for astronomical use.

Despite this fact, albeit some considerations and plans occurred, the astronomical X-ray telescopes flown so far on satellites mostly used the Wolter 1 type optics. However, it was used in several rocket experiments in the past, and in addition to that, it was proposed and discussed for several satellite experiments. Different situation is in the laboratory where $\mathrm{KB}$ systems are in frequent use, for example, at synchrotrons.

In contrast to the single double-plate system, the image of a point-like source starts to become increasingly extended in size as the number of plates involved increases. Wolter type I telescopes bend the incident ray direction two times in the same plane whereas the two bending in KirkpatrickBaez systems occur in two orthogonal planes, which for the same incidence angle on the primary mirror requires a longer telescope (Aschenbach, 2009 [79]).

3.2. KB-Early Stages. As already mentioned, Kirkpatrick and Baez (1948 [2]) have proposed an arrangement of concave mirrors to produce real images of extended objects with incidence at small grazing angles. They have considered both elliptical and parabolic profiles. The application in construction of an X-ray microscope was proposed, as well as focusing of electrons and other particles exhibiting external total reflection. It is worth to mention that their paper includes figures (and relevant discussion) with optics arrangement based on two reflectors/reflections but also on three of them.

The studies on focusing X-rays by mirror started already before the first published KB paper (Kirkpatrick and Baez, 1948 [2]), for example, one should mention the work of Ehrenberg (1947 [80]), who reported on efforts of formation of X-ray line images by reflection of X-rays from a point source at a gilded glass plate which had been bent to form an appropriate circular cylinder.

3.3. KB Systems in Astronomical Applications. Fisher and Meyerott (1966 [81]) have built another type of system with one-dimensional focusing which is composed of reflecting parabolas of translation that is, in a $\mathrm{KB}$ arrangement. $\mathrm{A}$ $\mathrm{KB}$ system of one-dimensional (1D) focusing was flown successfully in a sounding rocket by Gorenstein et al. (1971 [82]); during this flight, it was possible using the system to reconstruct an X-ray image of the Cygnus Loop based on the scans.

As an alternative to Wolter optics-based instruments, Van Speybroeck et al. [83] designed several telescope KB configurations that focus the $\mathrm{X}$ rays with sets of two orthogonal parabolas of translation. According to Van Speybroeck et al. [83] the crossed parabola systems should find application in astronomical observations such as high-sensitivity surveys, photometry, and certain kinds of spectroscopy where a large effective area is the most important factor rather than high angular resolution.
The design of a Kirkpatrick-Baez grazing-incidence Xray telescope was proposed and discussed by Kast (1975 [5]) for using to scan the sky and to analyze the distribution of both properly reflected rays and spurious images over the field of view. He has shown that in order to obtain maximum effective area over the field of view, it is necessary to increase the spacing between plates for a scanning telescope in comparison to a pointing telescope. Spurious images are necessarily present in this type of lens, but they can be eliminated from the field of view by adding properly located baffles or collimators.

X-ray telescopes suggested by Kirkpatrick and Baez (1948 [2]) have several advantages over other types of X-ray telescopes for a general sky survey for low-energy X-ray sources. Although their angular resolution for axial rays is worse if compared with telescopes using successive concentric figures of revolution, they can be constructed more easily and have greater effective area [84], note that more recent papers give different observations, see [85]. The design of KirkpatrickBaez-type telescopes has been discussed by several authors (e.g., Van Speybroeck et al., 1971 [84], Gorenstein et al., 1973 [86], Weisskopf. 1973 [87]), and results have been reported from several experiments using one-dimensional focus from a single set of plates (Gorenstein et al., 1971 [82], Catura et al., 1972 [88], Borken et al., 1972 [89]).

These analyses were carried out during the (HEAO-A) study of the large-area collector experiment for the High Energy Astronomy Observatory, Mission A spacecraft. This analysis was not specific to the HEAO-A instrument but can be applied generally to any one- or two-dimensional telescope of the Kirkpatrick-Baez type. The HEAO-A telescope $\mathrm{KB}$ design consisted of four separate quadrants of nested plates with a total aperture of about $75 \times 75 \mathrm{~cm}$ and a focal length of $6.55 \mathrm{~m}$, giving a ratio of half-aperture to focal length of $Y_{0} / F=0.057$. The plate length was $41 \mathrm{~cm}$ giving $\mathrm{LIF}=0.063$. The plate thickness $t$ was $0.18 \mathrm{~cm}$ giving a ratio $t / L=0.0044$. The detector had a square field of view of 20 by $20 \mathrm{~mm}$ (Kast 1975 [5]).

A method for optimizing the on-axis resolution of a $\mathrm{KB}$ reflecting element was presented and shown to be effective by Cohen (1981 [90]). Using the described procedure, the location of each of the 175 support points can be determined well by a computer assisted structural-optical software package before the actual assembly of each telescope unit. This automated procedure enabled to determine the optical characteristics of an entire telescope module consisting of seventy mirrors.

3.3.1. One-Dimensional KB System Used in Rocket Experiments. One-dimensional, and later two-dimensional (full), $\mathrm{KB}$ systems were flown in the past on several sounding rockets in order to achieve X-ray images of various celestial objects.

$\mathrm{X}$-ray emission from the supernova remnant known as the Cygnus Loop or Veil Nebula was observed using a onedimensional $\mathrm{KB}$ system in the energy range of 0.2 to $1 \mathrm{keV}$ from an attitude controlled Aerobee 170 sounding rocket that was launched on 26 June 1970 from the White Sands Missile Range (Gorenstein et al., 1971 [6]). 


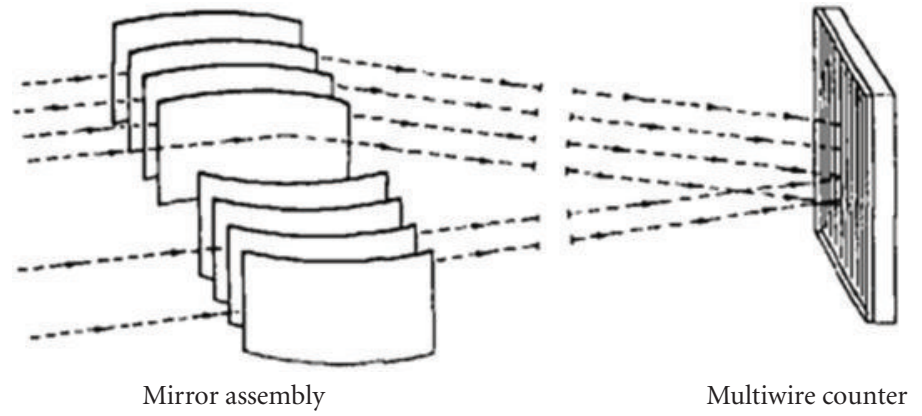

Figure 10: The one-dimensional KB optics used on rocket experiments (Gorenstein et al., 1971b [6]).

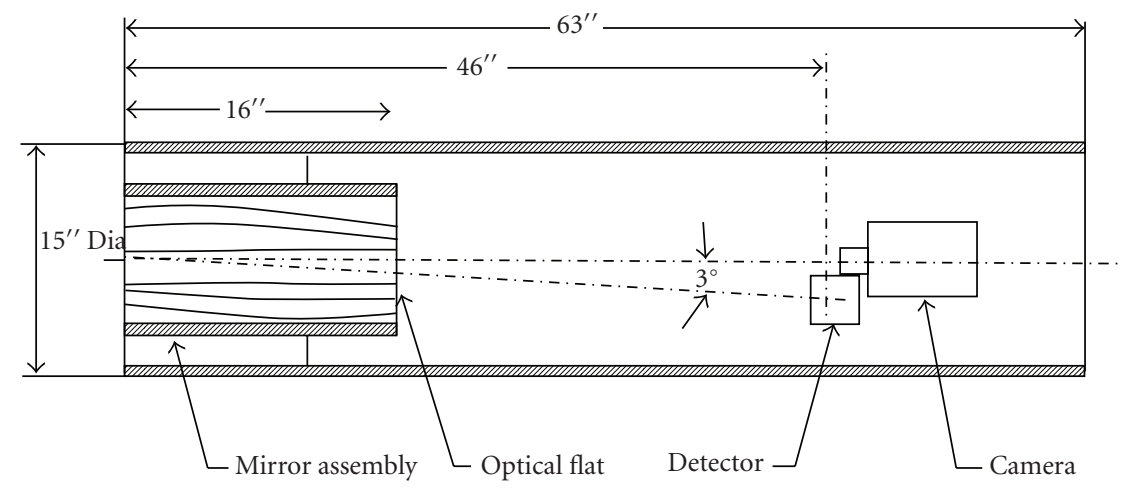

FIGURE 11: Schematic view of the KB telescope rocket payload (Gorenstein et al., 1971 [6]).

For that time, it was a rather unique instrument system, which focused X-rays by means of grazing incidence reflection, with the demonstration of advantages of focus compared to nonimaging systems used before, namely, better angular resolution and an improved ratio of signal to noise because the focused image is considerably smaller than the collecting area. Hence, the detector can be small and less influenced by cosmic ray effects.

The selection of KB optics was justified by the fact that the achievement of large sensitivity and a large field of view (FOV) was the main goal, not the angular resolution, due to the fact that the observational time available on sounding rockets in a scanning mode was very limited, to just few minutes.

The instrument consisted of a KB type collector that focused radiation in one dimension upon a multielement $\mathrm{X}$ ray detector in the focal plane (Figure 10). The collector (KB module) had eight nested reflecting plates whose dimensions were 20 by $40 \mathrm{~cm}$ and was symmetric about the central plane. Each plate was curved slightly to approximate a parabola in one dimension and focused to a common line. The overall field of view of the instrument was 2 deg along the direction of focusing and $9 \mathrm{deg}$ along the perpendicular direction. The eight reflecting surfaces were commercial 1$\mathrm{mm}$-thick float glass over coated with an evaporated layer of $150 \mathrm{~nm}$ of chromium for improved X-ray reflectivity at short wavelengths and were reinforced by a steel backing. The focal plane detector was a pair of four-wire proportional counters with a polypropylene entrance window of 1.3 microns.

Figure 11 shows a schematic view of the rocket payload (Gorenstein et al., 1971 [6]). The plates were fabricated from 0.040 inch sheets of commercial float glass measuring $8 \times$ 16 inch. A 0.020 inch steel backing was bonded to the glass with RTV adhesive for mechanical strength. Following a procedure used by the Columbia University group in a different type of collector, a $150 \mathrm{~nm}$ layer of chromium was evaporated onto the glass surface by a commercial manufacturer. The resulting surface retains the original smoothness of the glass and has improved reflection properties at the shorter wavelengths. The reflecting plates were mounted inside of an aluminium box and constrained to follow the correct parabolic curves by a set of accurately placed contact pins at the top and bottom. A serious limitation to most commercial varieties of float glass available at that time was that although the surfaces are smooth, they were not free of ripples, but even then the instrument delivered valuable results.

3.3.2. Two-Dimensional KB Systems Used in Rocket Experiment. After initial rocket experiments with one-dimensional $\mathrm{KB}$ systems, a soft X-ray imaging system consisting of a large area focusing (full, i.e., for two-dimensional imaging) $\mathrm{KB}$ optics and a position sensitive proportional counter was constructed for cosmic X-ray observations from a sounding rocket (Gorenstein et al. 1975 [91]). It was the first instrument obtaining non-solar celestial X-ray images. 
The focusing collector consisted of two successive arrays of reflecting plates shaped in the form of nested parabolas. The angular resolution of the system was $3 \prime$ over a field of 1.50 degrees. The detector was a two-dimensional device capable of a spatial resolution of about one millimetre for photon energies exceeding $0.25 \mathrm{keV}$.

As already mentioned, Kirkpatrick and Baez first proposed the two successive orthogonal X-ray reflections from slightly curved plates, which could be used to form X-ray images. Van Speybroeck et al. [84] considered the optical properties of a nested array of orthogonal parabolas of translation that form images by this method. They derived some general geometric relations for achieving optimum resolution that are applicable to KB imaging devices. For radiation on the axis, the resolutions theoretically perfect in one dimension and only about a second of arc in the other. For radiation off axis the resolution degrades as the first power of the angle. In practice, alignment errors and imperfections in the reflecting plates themselves have in the reported KB case (Gorenstein et al., 1975 [91]) at least resulted in resolution of a few minutes of arc everywhere within the field of view.

One of the principal advantages of this design was that commercially available float glass without further polishing could be used as the reflecting surfaces. The fine polishing is usually the most time consuming and costly process in telescope construction if the float glass sheets are used, then the polishing of reflecting surfaces is achieved essentially without effort. Of course, considerable effort had to be applied to the problems of forming the reflecting surfaces into the correct geometry, but this was of a lesser magnitude. The active glass surfaces were coated with $50 \mathrm{~nm}$ of gold stabilized by a $50 \mathrm{~nm}$ undercoat of chromium.

The reflecting plates in this experiment were housed in a rectangular box $25 \times 40 \mathrm{~cm}$ which was strengthened by four support rings. The effective collecting area of the device was determined by ray-tracing analysis using previously measured values of X-ray reflectivity versus angle of incidence. The angular resolution, about three minutes of arc in this case, was determined by the extent to which the authors succeeded in superimposing the images from the various plates. The system had a focal length of $180 \mathrm{~cm}$ and 25 front $\left(10^{\prime \prime} \times 20^{\prime \prime} \times 0.1^{\prime \prime}\right)$ and 18 rear $\left(14.55^{\prime \prime} \times 20^{\prime \prime} \times\right.$ $\left.0.1^{\prime \prime}\right)$ plates. The total geometric area was $1000 \mathrm{~cm}^{2}$ and the effective area $264 \mathrm{~cm}^{2}$ for $4.4 \mathrm{~nm}$ (Gorenstein et al., 1975 [91]).

3.4. $K B$ as a Segmented Mirror. Segmentation can also be applied, to the Kirkpatrick-Baez (KB) array of stacked orthogonal parabolic reflectors (Figure 12). As shown in Figure 13, a large KB mirror can be segmented into rectangular modules of equal size and shape (Gorenstein et al., 1996 [7]). A segmented KB telescope has the advantage of being highly modular on several levels. All segments are rectangular boxes with the same outer dimensions. Along a column, the segments are nearly identical and many are interchangeable with each other. All reflectors deviate from flatness only slightly. On the other hand, the Wolter

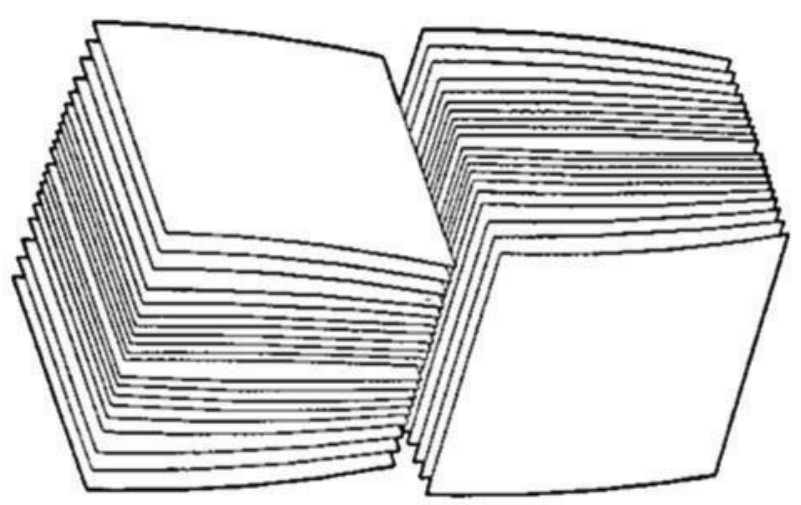

FIGURE 12: Kirkpatrick-Baez mirror consisting of orthogonal stacks of reflectors. Each reflector is a parabola in one dimension (Gorenstein et al., 1996 [7]).

reflectors are highly curved in the azimuthal direction and the curvature varies over a wide range. Furthermore, within a segment, the KB reflectors themselves can be segmented along the direction of the optical axis (Gorenstein 1998 [92]).

3.5. KB in Astronomical Telescopes: Recent Status and Future Plans. First attempt to create an astronomical KB module with silicon wafers was reported by Joy et al. [93]. They have constructed a test KB module based on new material/substrate, namely, silicon wafers. The telescope module consisted of 94 silicon wafers with diameter of $150 \mathrm{~mm}$, uncoated, with thickness of $0.72 \mathrm{~mm}$. The device was tested both in optical and X-rays, with measured FWHM of 150 arcsecs, dominated by large-scale flatness. It should be noted that the surface quality and flatness of Si wafers improved essentially over the time.

The recent efforts in future larger and precise imaging astronomical X-ray telescopes require reconsidering both the technologies as well as designs. The future large X-ray telescopes require new light-weight and thin materials/substrates such as glass foils and/or silicon wafers. Their shaping to small radii, as required in Wolter designs, is not an easy task. While the KB arrangements represent a less laborious and hence less expensive alternative.

The use of $\mathrm{KB}$ arrangement for IXO project (the proposed joint NASA/ESA/JAXA International X-ray Observatory) was suggested and investigated by Marsikova et al. [8] and more recently by Willingale and Spaan, 2009 [85]. These investigations indicate that if superior quality reflecting plates are used and the focal length is large, angular resolution of order of a few arcsec can be achieved (Figure 16). Recent simulations further indicate that in comparison to Wolter arrangement, the KB optics exhibit reduced on axis collecting area but larger field of view, at comparable angular resolution (Willingale and Spaan, 2009 [85]).

Comparison of Wolter and $\mathrm{KB}$ optical arrangement in astronomical X-ray telescope is summarized in Table 2 (Marsikova et al., 2009 [8]). Note that in order to achieve the comparable effective area, the focal length of KB system 
TABLE 2: Comparison of Wolter and KB optical arrangement in astronomical X-ray telescope (Marsikova, 2009 [8]).

\begin{tabular}{lcc}
\hline & KB & W \\
\hline Type of optics & Parabolic-parabolic planar & Parabolic-hyperbolic rotational \\
Number of reflections & 2 & 2 \\
Focal length aperture & $20 \mathrm{~m}-913 \times 913 \mathrm{~mm}$ & $10 \mathrm{~m}-$ dia $913 \mathrm{~mm}$ \\
& $40 \mathrm{~m}-1826 \times 1826 \mathrm{~mm}$ & $20 \mathrm{~m}-$ dia $1826 \mathrm{~mm}$ \\
First mirror & $134 \mathrm{~mm}$ from axis & $134 \mathrm{~mm}$ from axis \\
& $268 \mathrm{~mm}$ from axis & $398 \mathrm{~mm}$ from axis \\
Numbers of mirrors & 420 & 788 \\
Length of substrate & 840 & $300 \mathrm{~mm}$ \\
Material substrate & $300 \mathrm{~mm}$ & Glass \\
Surface & Silicon & Gold \\
\hline
\end{tabular}
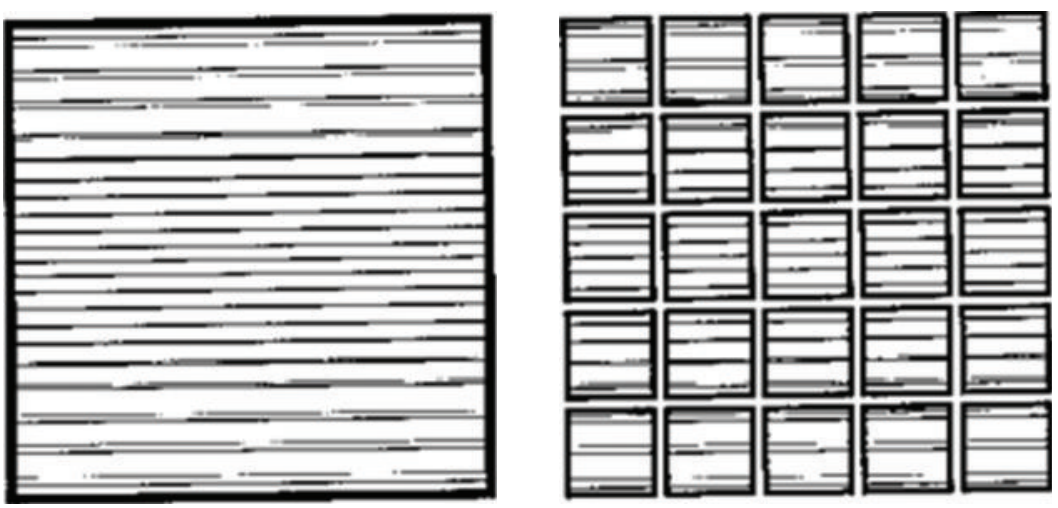

FIGURE 13: A large KB mirror can be segmented into rectangular modules of equal size and shape (Gorenstein et al., 1996 [7]).

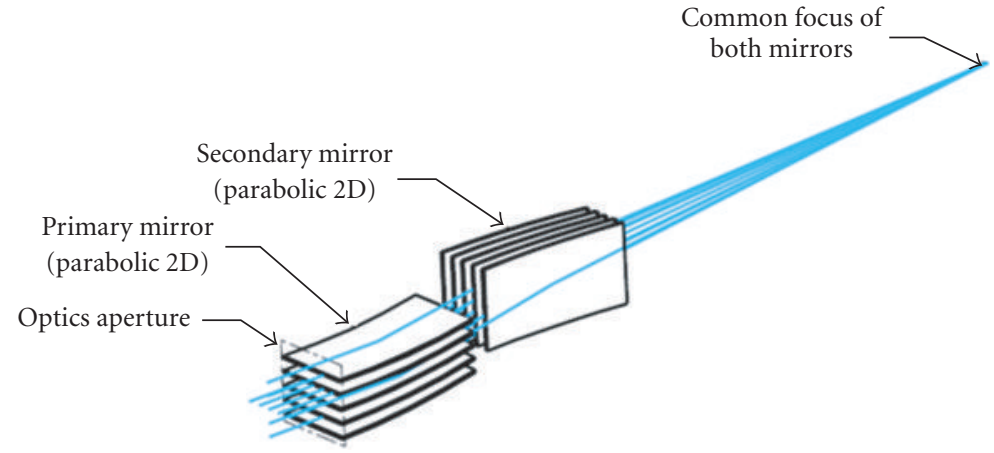

FIGURE 14: The principle of KB MFO telescope (Marsikova, 2009 [8]).

is required to be about twice of the focal length of Wolter system. The principle of MFO optics in KB arrangement is shown in Figure 14.

We note a very important factor and that is the ease (and hence, reduced cost) of constructing highly segmented modules based on multiply nested thin reflecting substrates if compared with Wolter design. While for example, the Wolter design for IXO requires the substrates to be precisely formed with curvatures as small as $0.25 \mathrm{~m}$ the alternative
$\mathrm{KB}$ arrangement uses almost flat or only slightly bent sheets. Hence the feasibility to construct $\mathrm{KB}$ module with required 5 arcsec FWHM at a affordable cost is higher than those for Wolter arrangement.

The advanced KB telescopes based on MultiFoil Optics (MFO) approach (X-ray grazing incidence imaging optics based on numerous thin reflecting substrates/foils, for more details, see [33]. The distinction between MFO and other optics using packed or nested mirrors is that MFO is based 
TABLE 3: Basic parameters of MFO KB modules assembled in 2009 (Marsikova, 2009 [8]).

\begin{tabular}{|c|c|c|c|}
\hline & KB 001 & KB 003 & KB 004 \\
\hline Shape of substrates: & Elliptic & Parabolic & Elliptic \\
\hline Focal length $[\mathrm{m}]$ : & 16 & 20 & 16 \\
\hline Aperture $[\mathrm{mm}]$ : & $100 \times 50$ & $100 \times 50$ & $100 \times 50$ \\
\hline Number of profiles: & 2 & 11 & 2 \\
\hline Number of substrates: & 6 & 33 & 6 \\
\hline Size of substrates $[\mathrm{mm}]$ : & $100 \times 100 \times 525$ & $100 \times 100 \times 525$ & $100 \times 100 \times 525$ \\
\hline Surface: & $\mathrm{Au}$ & $\mathrm{Au}$ & $\mathrm{Au}$ \\
\hline Skeleton/house: & $\mathrm{Al}$ & $\mathrm{Al}$ & $\mathrm{Al}$ \\
\hline
\end{tabular}

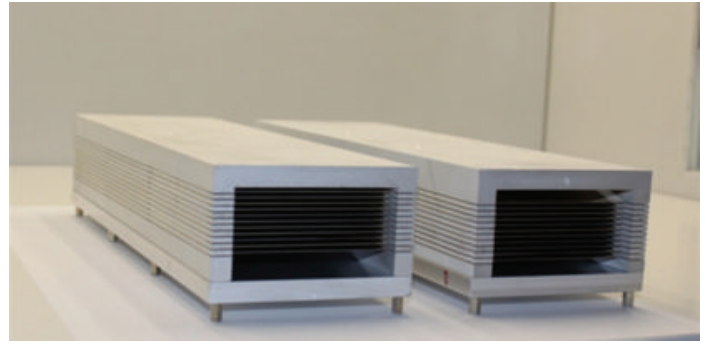

FIGURE 15: Laboratory samples of advanced KB MFO modules designed and developed at Rigaku Innovative Technologies Europe (RITE) in Prague (Marsikova, 2009 [8]). A summary of KB modules constructed so far is given in Table 3. The photograph shows the KB 003 module.

on numerous and very thin (typically less than $0.1 \mathrm{~mm}$ ) substrates. The following $\mathrm{KB}$ test modules were recently designed and constructed at Rigaku Innovative Technologies Europe (RITE) in Prague.

(i) Advanced technologies of Si substrates shaping were investigated and developed. Suitable substrates for Xray mirrors are supposed to be Si wafers because of their parameters.

(ii) Model based on raytracing (11 profiles)?.

(iii) Two sets of mirrors from Si chips $100 \times 100 \times$ $0.525 \mathrm{~mm}$ (Figure 15).

(iv) Total optics length $600 \mathrm{~mm}$, aperture $40 \times 40 \mathrm{~mm}$.

3.6. KB Microscopes. Apart from astronomical telescopes, there is a wide application of $\mathrm{KB}$ optics in various laboratory applications. The X-ray microscopy represents one of most important application.

McGee was the first who successfully demonstrated the use of crossed spherical reflectors in an X-ray microscope (McGee, 1957 [94]). Numerous applications and further improvements of KB systems in X-ray microscopes appeared in the following years. In this application, achievement of a very fine angular and spatial resolution is the most important goal.

3.6.1. Advanced KB Systems for X-Ray Microscopy. A fullfield hard X-ray microscope has the potential to observe nanostructures inside relative thick samples that cannot be observed by a transmission electron microscope. Consequently, it promises to be a powerful tool in fields such as material science and biology. The spatial resolution of a full-field X-ray microscope with a Fresnel zone plate (FZP) reaches $30 \mathrm{~nm}$ in the hard $\mathrm{X}$-ray region. However, chromatic aberration is an inevitable disadvantage of FZPs. To realize achromatic hard X-ray imaging, an imaging system with total-reflection X-ray mirrors is required. It is difficult to develop an X-ray mirror imaging system that can form an $\mathrm{X}$-ray image with a sub-100 $\mathrm{nm}$ resolution. Wolter optics is a promising imaging system, but, on the other hand, Wolter optics that are sufficiently accurate to realize diffractionlimited resolution have yet to be fabricated because it is very challenging to figure an axially symmetric aspherical shape, even using ultra-precision machining and measurements. That is why Kirkpatrick-Baez (KB) optics was actively studied for hard X-ray nanofocusing. Figuring elliptical mirrors is comparatively easy because their shapes are nearly planar; therefore, elliptical mirrors with a figure accuracy of $2 \mathrm{~nm}$ are feasible even using existing techniques. However, it should be noted that an optical system containing only an elliptical mirror cannot satisfy the Abbe sine condition and it suffers from comatic aberration.

The KB system according to Kodama et al. [10], termed advanced Kirkpatrick-Baez (AK-B) optics, consists of two pairs of hyperbolic and elliptic mirrors as shown in Figure 18(a), which are called as the AK-B type I microscope. The two dimensional configuration shown in Figure 18(b) is similar to the Wolter type I microscope configuration. The horizontal image is formed by the first and the fourth mirrors, and the vertical image by the second and the third mirrors. The combination of the hyperbolic and elliptic mirrors corrects the astigmatism, and the two mirror pairs can reduce the obliquity. In another configuration of the AKB (type II AK-B) the hyperbolic and elliptic mirrors were alternated, that is, the horizontal image is formed by the first and the third mirror and the vertical image by the second and the fourth mirror. However, the type II configuration generally has greater optical loss in the mirror assembly than type I. The spatial response of the microscope has been measured by X-ray backlighting a fine grid with laserplasma $X$ rays. A spatial resolution of better than $3 \mathrm{~nm}$ was reported with $2.5-\mathrm{keV} X$ rays over the field of $800 \mathrm{~nm}$ at a magnification of 25. This microscope was applied for laser implosion experiments, resulting in high-resolution images of the compressed cores. 

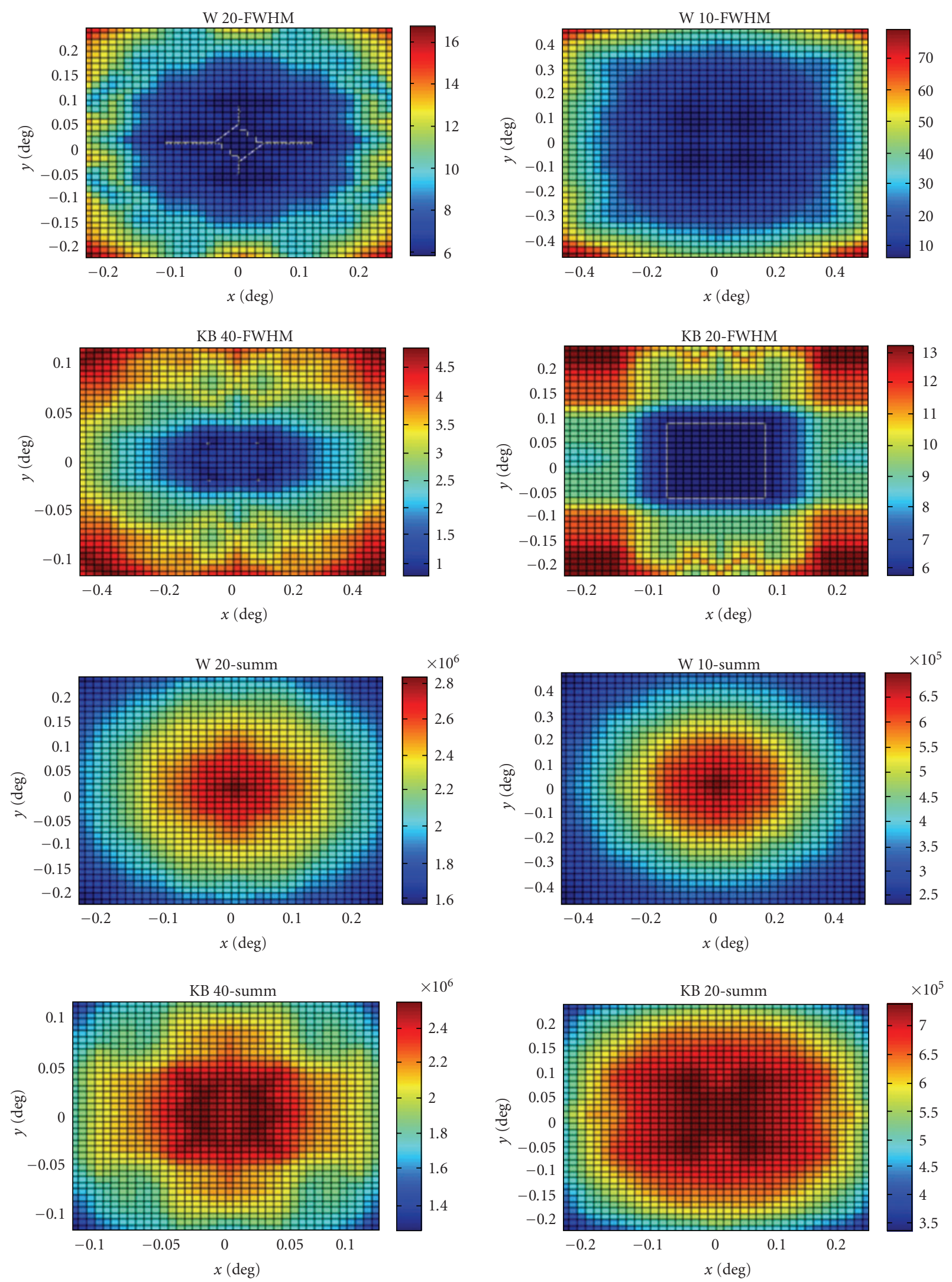

FiguRE 16: Comparison of focal images between KB and Wolter system (adopted from Marsikova, 2009 [8]). The upper 4 panels illustrate the FWHM in arcsecs, the bottom 4 panels the focal peak intensity. 


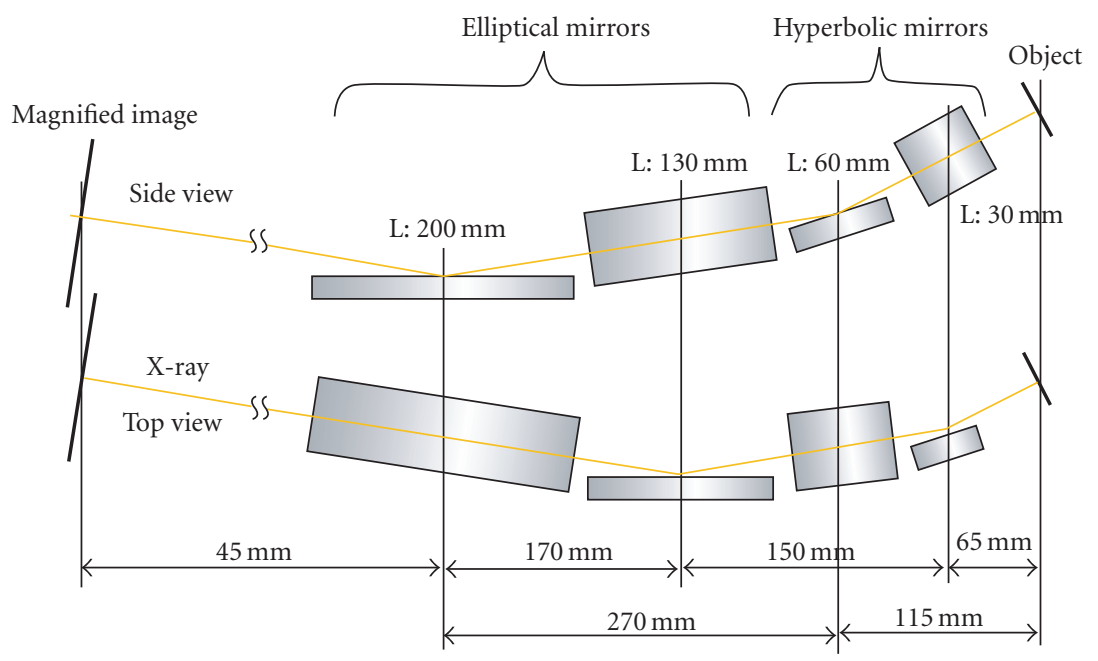

FIgURe 17: An Advanced KB system with 4 mirrors according to [9].

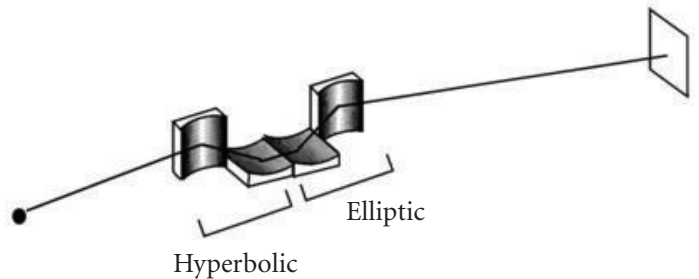

(a)

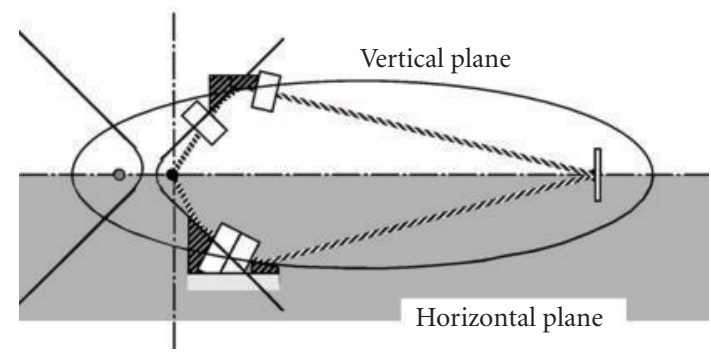

(b)

FIgURE 18: Schematic diagram of an AK-B microscope according to Kodama et al., 1996 [10]: (a) arrangement of the mirrors, (b) cross sections in the horizontal and the vertical planes.

However, AK-B optics containing four mirrors has to be aligned with high precision. This challenge needs to be overcome before AK-B optics can be utilized in practical applications. Matsuyama et al., 2009 [9], studied mirror alignment of AK-B optics with the goal of realizing a fullfield hard X-ray microscope with a resolution of better than $100 \mathrm{~nm}$. It is not currently known how accurately the four mirrors need to be aligned. In the study performed by Matsuyama et al., 2009 [9], the effect of one elliptical mirror and one hyperbolic mirror being misaligned on image quality was calculated using a wave-optics simulation. Based on these results, Matsuyama et al., 2009 [9], proposed a fourmirror alignment procedure for AK-B optics (Figure 17).
The mirrors for the AK-B microscope were made of fused-silica substrates. The surface figure of each mirror was measured with a Zygo interferometer. The deviation of the figure from a perfect surface (ether elliptic or parabolic) was less than $625 \mathrm{~nm}$. Taking into account the measured deviations of all the mirrors in a ray-trace calculation, the spatial resolution of the AK-B was found to be degraded by less than $0.7 \mathrm{~nm}$ (the half-width of the point-spread function) at the centre of the field of view.

3.6.2. Scanning X-Ray Fluorescence KB Microscopes. A scanning X-ray fluorescence microscope (SXFM) is an imaging tool with which the element distribution of a sample can be visualized using X-ray fluorescence generated by the focused hard X-ray irradiation of the sample. Because the excitation beam consists of hard $\mathrm{X}$ rays, there is no need to install samples under vacuum. In this microscopy, spatial resolution and sensitivity depend on, respectively, beam size and photon flux. In the sensitivity point of view, the combination of a synchrotron radiation source, which can generate the brightest X-ray, and KB mirrors, which have high focusing efficiency, is one of the most powerful focusing systems for a SXFM. In terms of spatial resolution, the previous reports regarding hard X-ray nanofocusing suggested that $\mathrm{KB}$ mirrors enable us to obtain a nanobeam having a full width at half maximum FWHM of better than $40 \mathrm{~nm}$. Owing to achromatic focusing using total reflection on a mirror surface, we can select the most efficient energy of X rays for various samples and experimental conditions.

An example of a high-spatial-resolution scanning Xray fluorescence microscope (SXFM) using KirkpatrickBaez mirrors was developed and reported by Matsuyama et al., 2006 [8]. As a result of two-dimensional focusing tests, the full width at half maximum of the focused beam was achieved to be $50 \times 30 \mathrm{~nm}^{2}$ under the best focusing conditions. The measured beam profiles were in good agreement with simulated results. Moreover, beam size was controllable within the wide range of $30-1400 \mathrm{~nm}$ by 


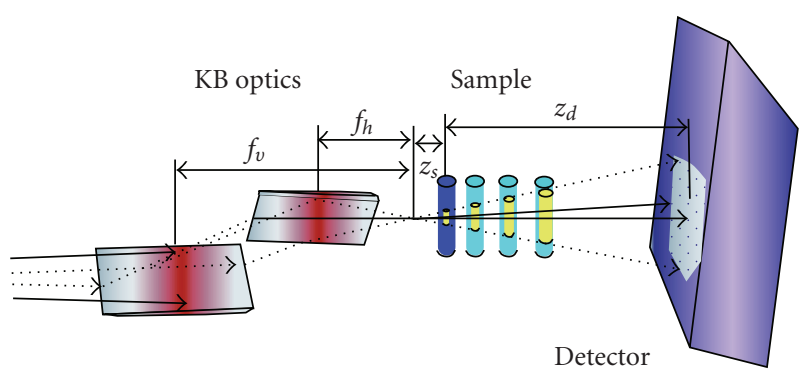

FIGURE 19: Scheme of the tomography setup using the divergent beam produced by Kirkpatrick-Baez optics (Mokso et al., 2007 [11]).

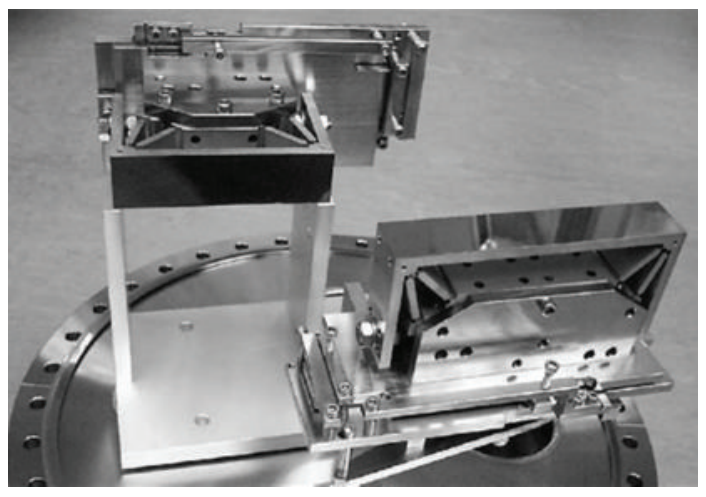

Figure 20: The modular K-B micro focusing mirror system is tested to mount on the 1400 rotatable CF flange (Chen et al., 2001 [12]).

changing the virtual source size, although photon flux and size were in a tradeoff relationship. To demonstrate SXFM performance, a fine test chart fabricated using focused ion beam system was observed to determine the best spatial resolution. The element distribution inside a logo mark of SPring-8 in the test chart, which has a minimum line width of approximately $50-60 \mathrm{~nm}$, was visualized with a spatial resolution better than $30 \mathrm{~nm}$ using the smallest focused X-ray beam, demonstrating the superior performance of KB optics.

3.7. KB in X-Ray Tomography. The improvement of spatial resolution triggered by a broad spectrum of materials science and biological applications is one main driving force toward innovative designs and techniques in imaging technologies that provide three-dimensional 3D information about the sample in a nondestructive manner (Mokso et al. [11]). Xray tomography is the oldest among them and still evolving. The use of Fresnel zone plates as objective lens has brought resolutions in the $100 \mathrm{~nm}$ range and is most applied in the soft X-ray regime for the study of thin and light materials such as single cells. The emerging field of coherent diffraction imaging, based on phasing a coherent Fraunhofer diffraction pattern, is expected, in the 3D imaging of tiny isolated objects, to overcome the resolution limit set by the Xray optical devices. A drawback of the improved spatial resolution is the corresponding decrease in field of view and sample size. Larger, millimetre-sized samples can be

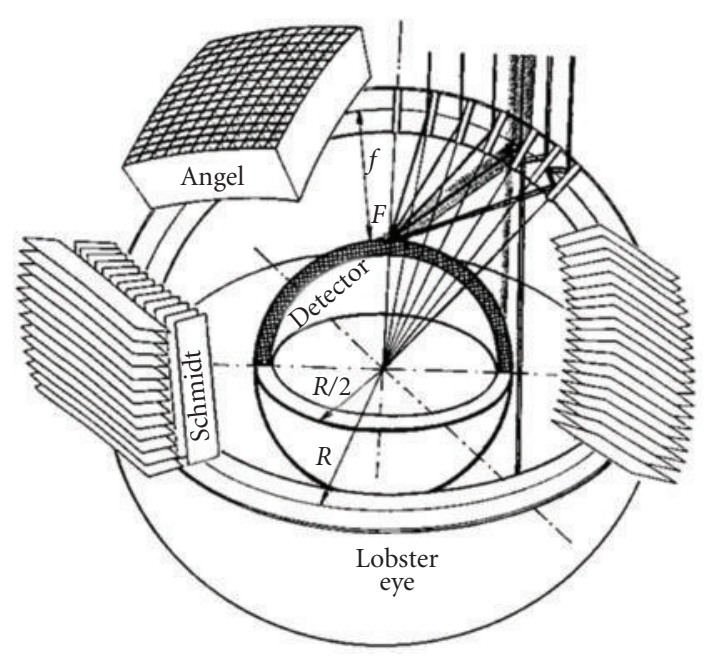

(a)
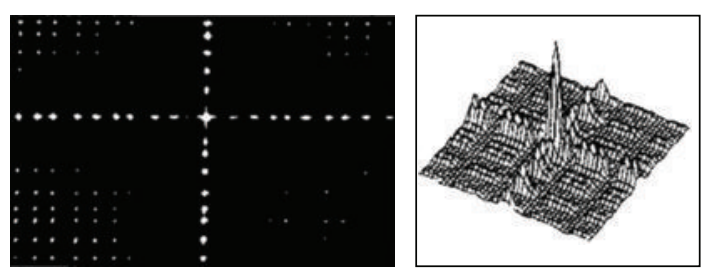

(b)

FIGURE 21: The schematic arrangement of the Lobster Eye type Xray optics (a) with real image of the Schmidt objective prototype in optical light (b left) and distribution of intensity on the focal sphere for a point-line source (computer ray-tracing, b right).

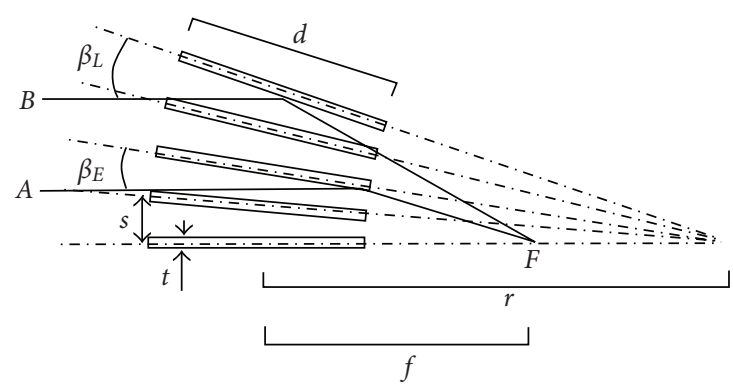

FIGURE 22: The schematic arrangement of the Lobster Eye type Xray optics used for simple equations derivation (Sveda, 2003 [15]).

investigated in 3D with parallel beam synchrotron radiation micro tomography, but in this case, the resolution is limited by detector technology to slightly better than $1 \mathrm{~nm}$.

A projection microscope that bridges the gap in terms of resolution and specimen size between these nanoscale and micro scale 3D imaging methods was suggested by Mokso et al., 2007 [11]. It is based on state-of-the-art focusing of hard X rays characterized by a large penetration power and depth of focus. This allows exploring nondestructively in a three-dimensional manner bulk material at the nanoscale. The high flux makes it adapted to fast imaging for dynamical studies. Further improvement of spatial resolution does not 


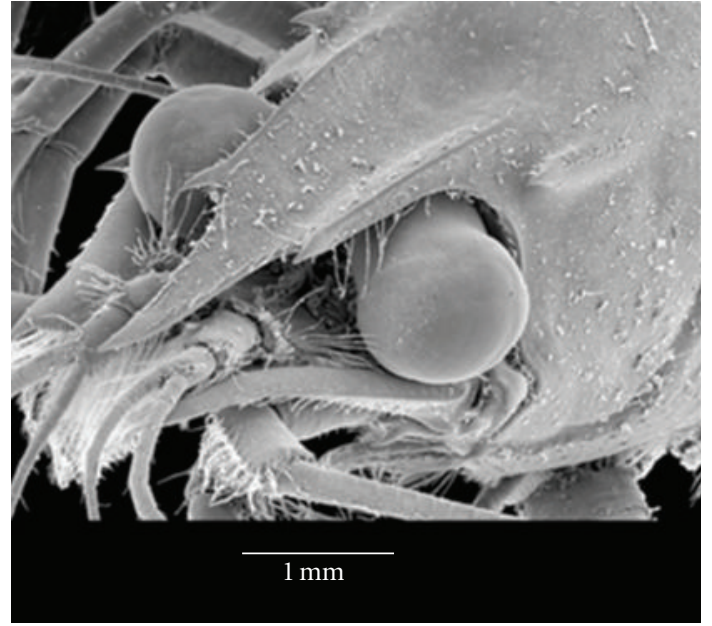

(a)

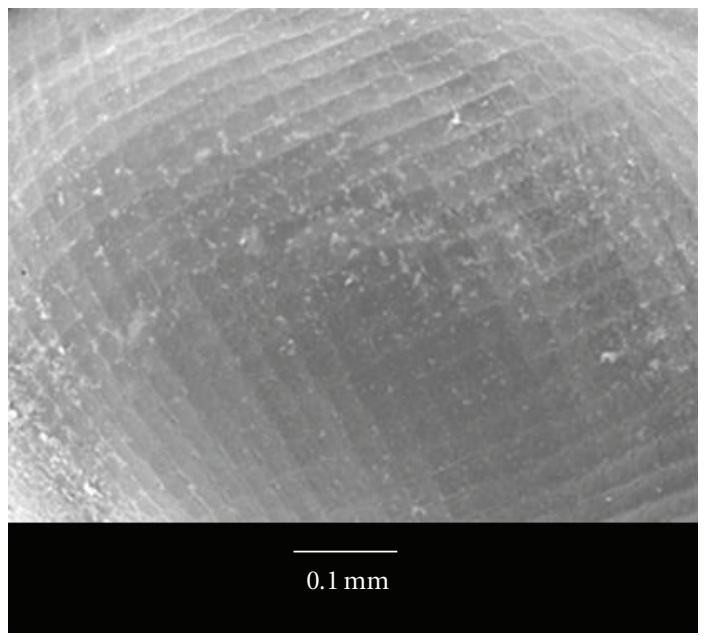

(c)

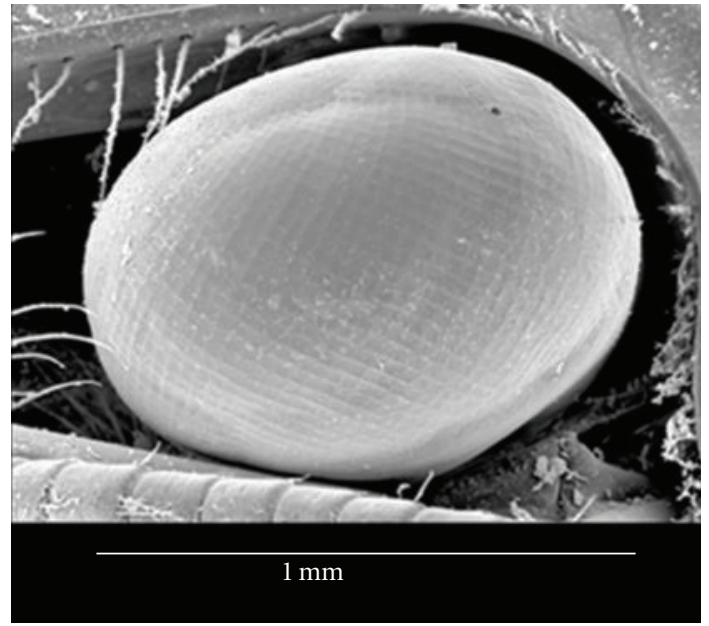

(b)

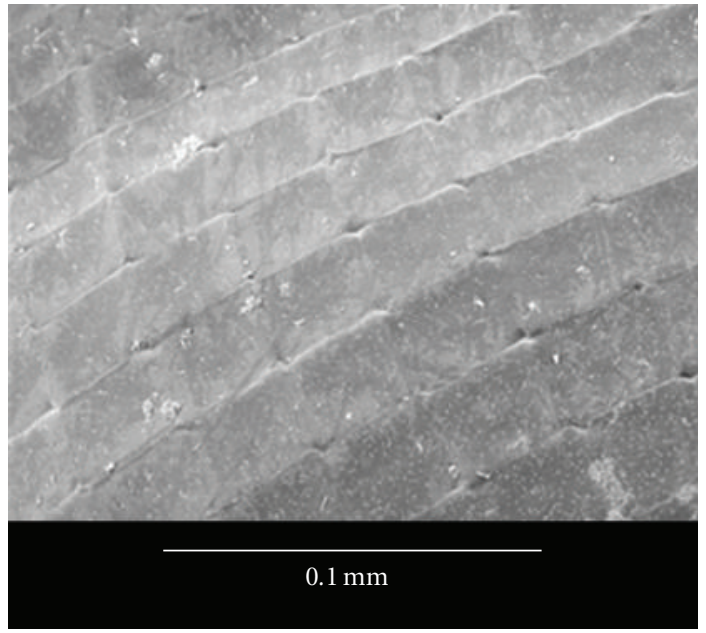

(d)

Figure 23: The $d$ of a crayfish with eyes, the crayfish eye (a, b), and the surface details (c, d).

necessarily imply a reduced efficiency as it is the case for imaging with Fresnel zone plates and high-resolution detectors. In the original tomography setup sketched on Figure 19, bent-graded multilayer's set in the Kirkpatrick-Baez (KB) geometry focus undulator radiation to a spot below $90 \mathrm{~nm}$ in both directions. The focusing device generates a spherical wave illumination of the sample.

3.8. KB Systems for X-Ray Microprobes. A synchrotron Xray microprobe having a submicron beam size based on the Kirkpatrick-Baez configuration was designed and constructed by using synchrotron radiation from a multiple wiggler for example, at the Photon Factory (Iida and Hirano, 1996 [95]). The described X-ray microprobe system is usable for spectroscopic and diffraction experiments. Though it is apparent that the $\mathrm{X}$-ray microprobe technique will be more effectively developed using a third-generation lowemittance synchrotron light source, the described sub- micron system is practically useful for material characterization, considering the deep X-ray penetration depth and the difficulty in preparing extremely thin samples. Furthermore, the improved Kirkpatrick-Baez optics will attain the higher photon flux and the smaller beam size with the thirdgeneration undulator radiation.

A K-B mirror system for microprobe was designed by Chena et al. [12], to achieve a spot size of $6.7 \times 2.9 \mathrm{~mm}^{2}$ and a gain of $6.2 \times 10^{3}$ in the EPU beam line, based on the following considerations: gain, efficiency, and focal spot size. This mirror system, which contains a sixth order polynomial corrected cylindrical horizontal micro focusing mirror (HMFM) and an eight-order polynomial vertical micro-focusing mirror (VMFM) can accumulate and micro focus the undulator source into a nearly perfect point image. Two 17-4 PH stainless steel bent K-B mirrors with the monolithic flexure-hinge and modular mounting designs have the following features: (1) compact and modular fabrication, (2) stability of bending mechanism without 
backlash, (3) appropriate for X-ray microfocusing system when mirrors have the central radii of curvature less than $10 \mathrm{~m}$, and (4) modular and independent.

X-ray microbeam optics capable to achieve micron spatial resolution and high focusing gain has received considerable interest. Microfocusing photon beam of any wavelength mainly on free optical principles: reflectivity, refractivity, and diffraction. Fresnel zone plate accurately represents X-ray microbeam optics according to the diffraction. By applying lithography and deposition techniques, a feasible zone plate in which the outmost zone has a width of about $20 \mathrm{~nm}$ can microfocus the X-ray of synchrotron radiation into a beam spot size of few $10 \mathrm{~nm}$ with efficiency exceeding $10 \%$. The compound refractive lenses that are fabricated from low- $\mathrm{Z}$ materials setup as a linear array of lenses have focal spots of few microns for a hard X-ray range.

Both K-B mirror system and tapered capillary optics can focus photon beams to achieve submicron spatial resolution based on the reflectivity. Selecting appropriate Xray microbeam optics involves considering the focal spot size, photon energy range, photon flux density, efficiency, and divergent angle. For X-ray scanning experiments, the focal spot size must be as small as possible to achieve spatial resolution. However, transmission image experiments prefer the photon flux density to the spot size so that a few micron focal spots are acceptable. Microfocusing systems in the EPU beam line of Synchrotron Radiation Research Centre (SRRC) that exploit the merits of microbeams for transmission image experiments in soft X-ray range were designed according to the primary consideration: photon flux density (Chen et al., 2001 [12]). Therefore, K-B focusing mirror systems was adopted to achieve the microfocusing function and maintain their higher efficiency and no chromatic aberration in contrast to Fresnel zone plates (Chen et al., 2001 [12]). To satisfy the strict requirements of X-ray microbeam optics, two high order polynomial corrected cylindrical mirrors, HMFM and VMFM, based on the K-B design were designed to focus the X-ray beam with nearly no aberration and then demagnify it onto a micron image (Figure 20).

3.9. Application of KB for Neutron Focussing. As shown, for example, by Ice at al., 2005 [96], Kirkpatrick-Baez neutron supermirrors can efficiently focus neutron beams into small areas with a maximum divergence that is limited by the mirror critical angle. The size of the focal spot is primarily determined by geometrical demagnification of the source and by figure errors in the mirror shape. Ray-tracing calculations show that high-performance Kirkpatrick-Baez super mirrors can preserve neutron-source brilliance when focusing down to tens of microns and can focus approximately two orders of magnitude greater power into 100 microns than it is practical without focusing. The predicted performance is near the theoretical limit set by the source brilliance. Ice et al. [96], described the phase space arguments, ray-tracing calculations and actual performance of an M3 super mirror system designed to produce a focal spot below 100 microns. Although their design was optimized for neutron polychromatic micro diffraction, the design principles are certainly widely applicable to a range of neutron science.

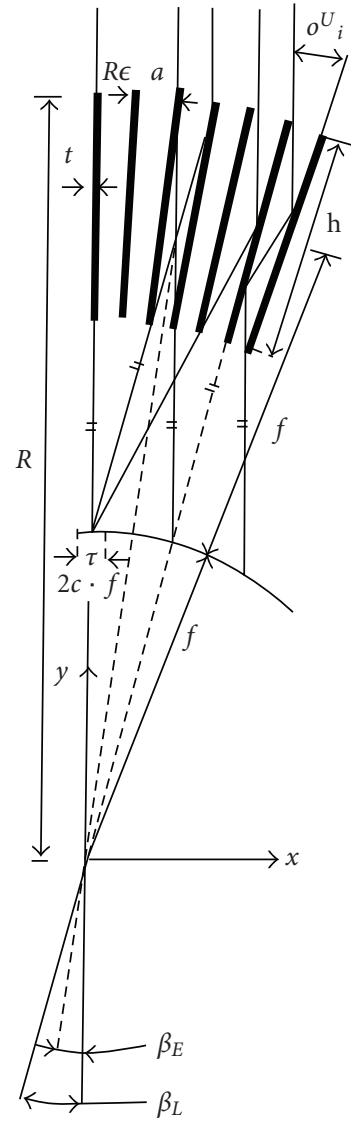

Figure 24: The X-ray reflections on the Schmidt X-ray lens.

\section{Lobster-Eye Wide-Field X-Ray Optics}

4.1. Introduction. The principle of lobster-eye type widefield X-ray optics was published by Angel (Angel, 1979 [60]). The full lobster-eye optical grazing incidence X-ray objective consists of numerous tiny square cells located on the sphere and is similar to the reflective eyes of macruran crustaceans such as lobsters or crayfish (Figures 21 and 23). An alternative arrangement was proposed by Schmidt 1975 [97] (Figures 21 and 22). Both these arrangements will be discussed in detail later.

The wide-field mirror modules offer advantageous application in astrophysics. The major scientific achievements of the X-ray astronomy in the past are closely related to the use of large X-ray imaging telescopes based mostly on the Wolter 1 X-ray objectives. These systems usually achieve excellent angular resolution as well as very high sensitivity, but are quite limited in the field of view available, which is less than 1 degree in most cases. However, the future of Xray astronomy and astrophysics requires not only detailed observations of particular triggers, but also precise and highly sensitive X-ray sky surveys, patrol and monitoring. The recently confirmed X-ray counterparts of Gamma Ray Bursters (GRBs) may serve as an excellent example. For recently in detail investigated GRB with precise localization accuracy, in almost all cases variable and/or fading X-ray 


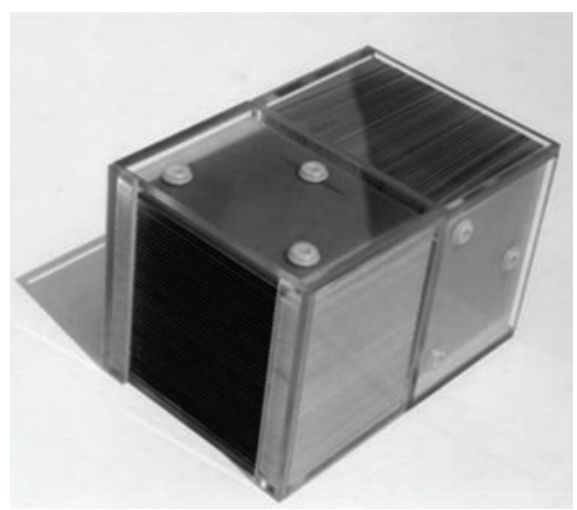

(a)

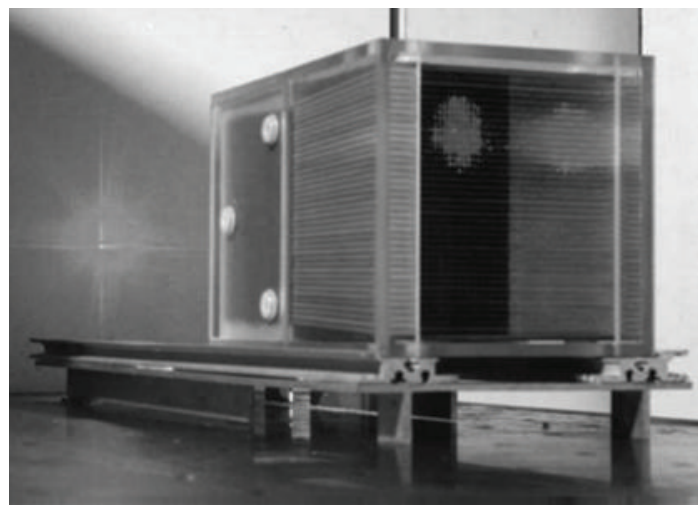

(b)

Figure 25: (a) The Schmidt objective test module, $100 \times 80 \mathrm{~mm}$ plates. (b) Optical tests of the Lobster eye objective from the (a).
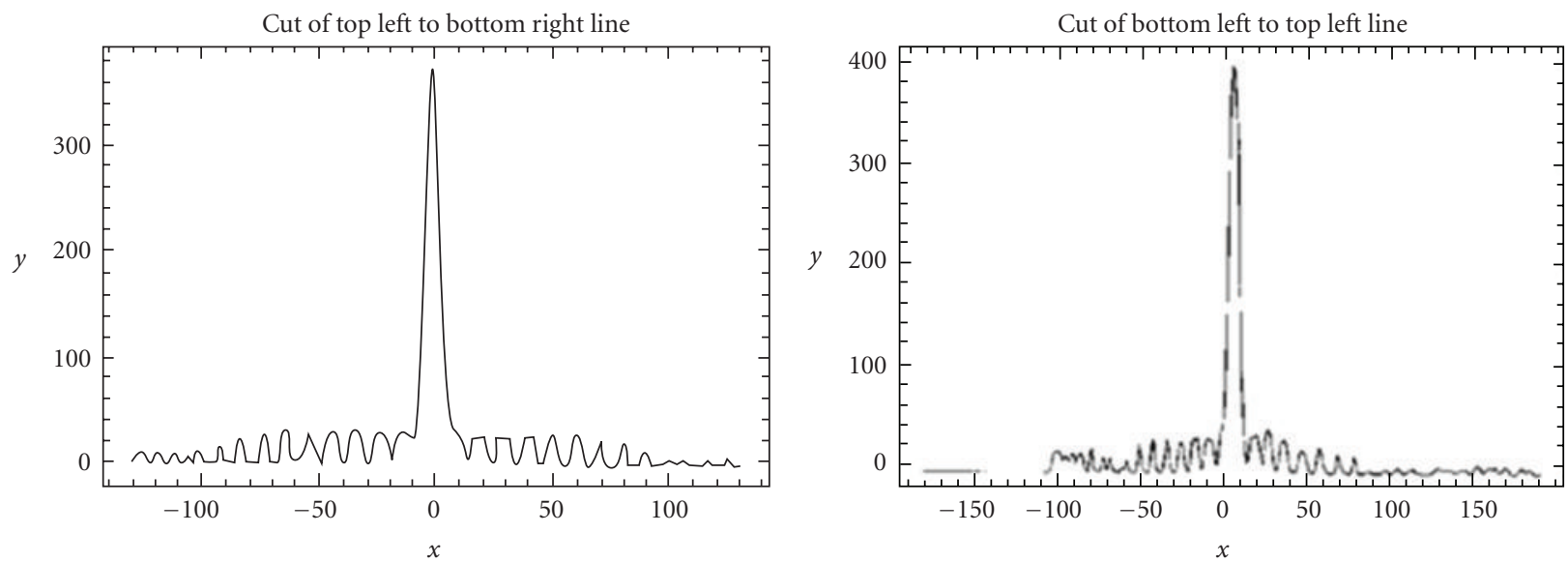

Figure 26: X-ray focal images of the Schmidt telescope prototype from Figure 25(a) at $1.5 \mathrm{~nm}$ taken in collaboration in the test facility of the X-ray astronomy group, University of Leicester, UK.

counterparts/afterglows have been identified. The X-ray identification of GRBs has lead to great improvements in study and understanding of these sources and especially has allowed identifications at other wavelengths due to better localization accuracy provided in X-rays if compared with gamma ray observations. Since most of GRBs seem to be accompanied by X-ray emissions, the future systematic monitoring of these X-ray transients/afterglows is extremely important. However, these counterparts are faint in most cases, hence powerful wide field telescopes are needed. An obvious alternative seems to be the use of wide field Xray optics allowing the signal/noise ratio to be increased if compared with nonfocusing devices. The expected limiting sensitivity of lobster eye telescopes is roughly $10^{-12} \mathrm{erg}^{-2} \mathrm{~s}^{-1}$ for daily observation in soft X-ray range. This is consistent with the fluxes detected for X-ray afterglows of GRBs. Furthermore, the wide field X-ray telescopes may play an important role in monitoring of faint variable $\mathrm{X}$-ray sources to provide better statistics of such objects (note, e.g., the occurrence of two faint fading X-ray sources inside the 


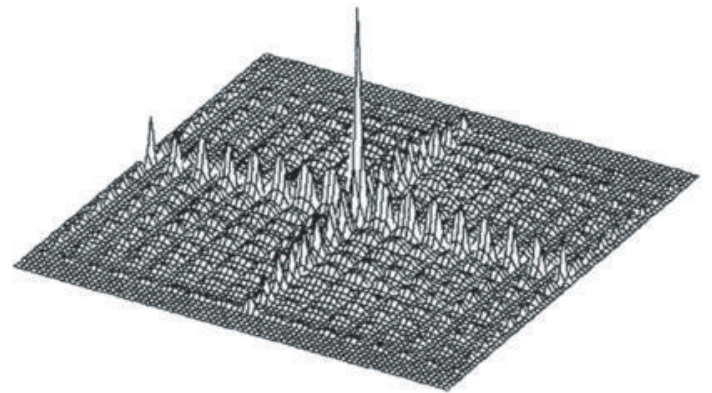

FIGURE 27: Intensity distribution in the focal plane $(40 \times 40 \mathrm{~mm}$ detector) according to mathematical ray-tracing for the identical test module and test arrangement as given above; the microroughness of reflecting surfaces are assumed to be $1 \mathrm{~nm}$.

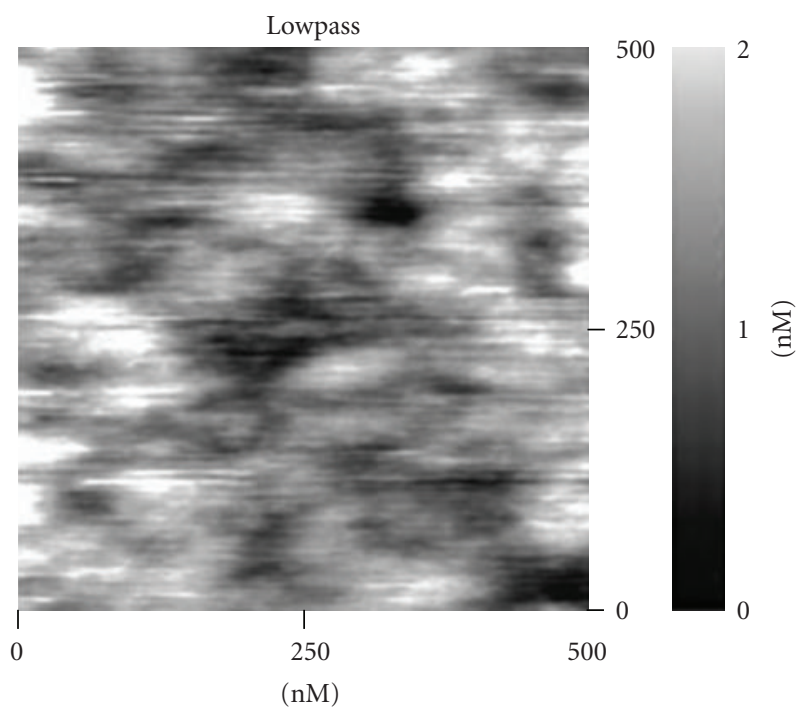

FIGURE 28: Tapping AFM images of the surface of the double-sided $\mathrm{X}$-ray reflecting flats taken in collaboration in the test facility of the Astronomical Observatory in Brera, Italy-the resulting micro roughness RMS is $0.3 \mathrm{~nm}$.

gamma ray box of GRB970616) as well as in other fields of $\mathrm{X}$-ray astrophysics. The recent hunting for faint fading X-ray afterglows of GRBs has indicated that there is a large number of faint and/or variable X-ray sources worth of detailed study.

The wide field lobster eye telescopes are expected to play an important role in future X-ray astrophysics missions and analyses. The advanced prototypes of lobster eye optics modules of various sizes and various arrangements confirm the justification of space applications of these innovative devices. Both very small $(3 \times 3 \mathrm{~mm}$ based on $0.03 \mathrm{~mm}$ foils spaced at $0.07 \mathrm{~mm})$ and large $(300 \times 300 \mathrm{~mm}$ based on $0.75 \mathrm{~mm}$ foils spaced at $10.8 \mathrm{~mm}$ ) LE Schmidt prototypes have been designed, developed and tested. Advanced technologies for additional surface layers have been investigated as well.

There have been many attempts to increase slightly the available wide field coverage of Wolter and analogous X-ray telescopes. To avoid any confusion, we suggest restricting the term "wide-field X-ray optics" only for optical systems with field of view $\gg 1$ degree while to use the term "narrow-field system" for systems with FOV $<1$ degree.

The angular resolution is a function of spacing between the reflecting plates and focal length. In Schmidt arrangement, the Lobster Eye consists of plates of thickness $t$, depth $d$ (Figure 22). Spacing between plate planes is $s$, focal length, radius $r$, focal point $F, \beta$ the angle between optical axis and focused photons in time of detection. Beam A (Figure 22) shows the situation, where the plate is fully illuminated and the crossection of the plate is maximal (effective reflection). Beam B is the last beam that can be reflected into the focal point. Each beam, which is further from the optical axis, reflects more than once (critical reflection). If reflected twice from the same set of plates, photon does not reach the focal point and continues parallel to the incoming photon direction (Sveda $2003[15]$ ).

If $t \ll s \ll d \ll f$ we can derive the following simple equations (Sveda 2003 [15] Inneman 2001 [98]), where $\alpha$ is the estimate of the angular resolution

$$
\begin{gathered}
f=\frac{r}{2}, \\
\beta_{E}=\frac{a-t}{d}, \\
\beta_{L}=2 b E, \\
\alpha \sim \frac{2 s}{r}=\frac{s}{f} .
\end{gathered}
$$

Detailed ray tracing simulations were performed for the LE modules designed and developed, for more details, see for, example, [15].

4.2. Schmidt Objectives. The lobster-eye geometry X-ray optics offers an excellent opportunity to achieve very wide fields of view. One-dimensional lobster-eye geometry was originally suggested by Schmidt (Schmidt, 1975 [53]), based upon flat reflectors. The device consists of a set of flat reflecting surfaces. The plane reflectors are arranged in an uniform radial pattern around the perimeter of a cylinder of radius $R$. X-rays from a given direction are focussed to a line on the surface of a cylinder of radius $R / 2$ (Figure 24). The azimuthal angle is determined directly from the centroid of the focused image. At glancing angle of X-rays of wavelength $1 \mathrm{~nm}$ and longer, this device can be used for the focusing of a sizable portion of an intercepted beam of X-ray incident in parallel. Focussing is not perfect and the image size is finite. On the other hand, this type of focusing device offers a wide field of view, up to maximum of half sphere with the coded aperture. It appears practically possible to achieve an angular resolution of the order of one tenth of a degree or better. Two such systems in sequence, with orthogonal stacks of reflectors, form a double-focusing device. Such device should offer a field of view of up to 1000 square degrees at moderate angular resolution.

It is obvious that this type of X-ray wide field telescopes could play an important role in future X-ray astrophysics. The innovative very wide field X-ray telescopes have been suggested based on these optical elements but have not been 


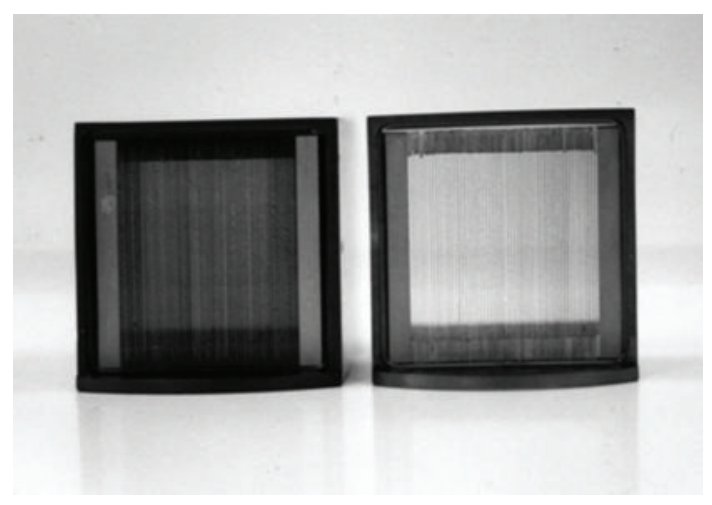

(a)

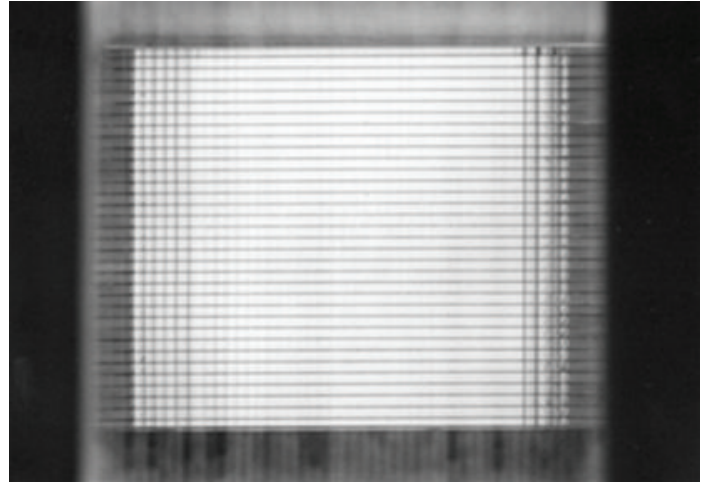

(b)

Figure 29: (a) The mini-Schmidt objective test modules based on two sets of $6023 \times 23 \mathrm{~mm}$ flats, $0.1 \mathrm{~mm}$ thick used for X-ray tests illustrated on Figures 31(a)-37). (b) The front view of the mini-Schmidt objective test module from (a).

flown in space so far (e.g., Gorenstein 1979 [99]). One of the proposals is the All Sky Supernova and Transient Explorer (ASTRE). This proposal also includes a cylindrical coded aperture outside of the reflectors, which provide angular resolution along the cylinder axis (Gorenstein 1987 [61]). The coded aperture contains circumferential open slits $1 \mathrm{~mm}$ wide in a pseudorandom pattern. The line image is modulated along its length by the coded aperture. The image is cross-correlated with the coded aperture to determine the polar angle of one or more sources. The field of view of this system can be, in principle, up to 360 degree in the azimuthal direction and nearly $90 \%$ of the solid angle in the polar direction. To create this mirror system, a development of double-sided flats is necessary. There is also potential for possible extending the wide field imaging system to higher energy by the use of multilayer or other coatings in analogy to those described for flat reflectors in the Kirkpatrick-Baez geometry.

First lobster-eye X-ray Schmidt telescope prototype consisted of two perpendicular arrays of flats (36 and 42 double-sided flats $100 \times 80 \mathrm{~mm}$ each). The flats are $0.3 \mathrm{~mm}$ thick and gold-coated (Inneman et al. 2002 [100], Inneman et al. 2002 [101]). The focal distance is $400 \mathrm{~mm}$ from the midplane. The FOV is about 6.5 degrees (Figures 25(a) and 25(b)). The results of optical and X-ray tests have indicated the performance close to those provided by mathematical modelling (ray-tracing). The X-ray test has been carried out in the test facility of the X-ray astronomy group, University of Leicester, UK (Figure 26). Another test modules of Schmidt geometry have been designed and developed, based on $0.1 \mathrm{~mm}$ thick gold-coated glass plates $23 \times 23 \mathrm{~mm}$ at $0.3 \mathrm{~mm}$ spacing. The aperture/length ratio is 80.60 such plates represent one module. Two analogous modules represent the $2 \mathrm{D}$ system for laboratory tests, providing focus to focus imaging with focal distances of 85 and $95 \mathrm{~mm}$. The innovative gold coating technique has improved the final surface micro roughness rms to $0.2-0.5 \mathrm{~nm}$ (Figure 28). Various modifications of this arrangement have been designed both for imaging sources at finite distances (for laboratory tests) as well as of distant sources (the corresponding double-focusing array has $f=250 \mathrm{~mm}$ and $\mathrm{FOV}=2.5 \mathrm{deg})$. In parallel, numerous ray-tracing simulations have been performed, allowing comparing theoretical and experimental results (Figures 27 and 29(b)).

Later on another test lobster eye modules have been constructed and tested both in visible light and in X-rays. As an example, we show X-ray test results for the mini-LE module. These results illustrate in detail the on-axis and offaxis imaging performance of the LE module tested.

The module is shown on Figure 29(a) and the test results including the experiment arrangement are in Figures 32(b) to $33,34,35,36$, and 37 Figure 31 (b) and 32(a) show the Xray measurement results of micro-LE X-ray module shown in Figure 45. For review of LE modules designed, constructed and tested see Table 4 . The X-ray tests on coated flats used in LE modules as reflectors are illustrated in Figures 30 and 31(a). The largest LE module maxi is shown in Figure 42 and the example of optical imaging test is in Figure 43.

All test experiments of the mini-LE schmidt module (illustrated on Figure 29(a)) in 31(b)-36(b) were done with the microfocus $\mathrm{X}$-ray tube (Bede microsource, $\mathrm{Cu}$ anode, $40 \mathrm{kV}, 100$ microamp) and the X-ray CCD Digital Camera (Reflex X16D3, 16 bit, DN > 30 000, $512 \times 512$ pixels, Back Illuminated CCD chip SITe, direct exposure).

4.3. Angel Objectives. Besides the Schmidt objectives described above, there is also an alternative based on slightly different arrangement, sometimes referred as two-dimensional lobster eye optics. The idea of two-dimensional lobster-eye type wide-field X-ray optics was first mentioned by Angel (Angel, 1979 [60]). The full lobster-eye optical grazing incidence $\mathrm{X}$-ray objective consists of numerous tiny square cells located on the sphere and is similar to the reflective eyes of macruran crustaceans such as lobsters. The field of view can be made as large as desired and good efficiency can be obtained for photon energies up to $10 \mathrm{keV}$. Spatial resolution of a few seconds of arc over the full field is possible, in principle, if very small reflecting cells can be fabricated at long focal lengths. 

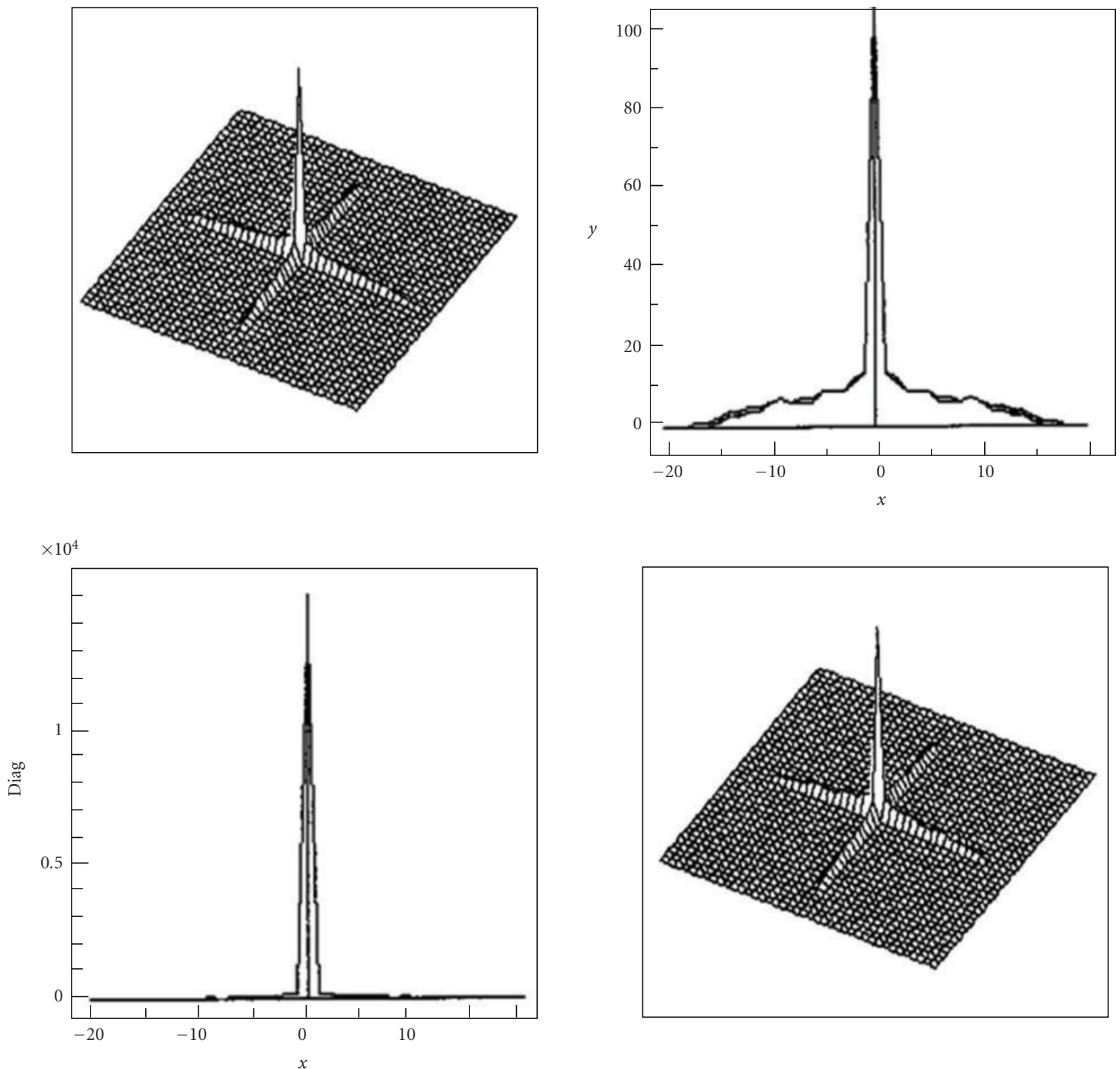

FIGURE 30: Intensity distribution in the focal plane $(15 \times 15 \mathrm{~mm}$ detector $)$ for the $23 \times 23 \mathrm{~mm}$ Schmidt objective, mathematical raytracing for micro roughness $\mathrm{rms}=1 \mathrm{~nm}$ and wavelength $=1 \mathrm{~nm}$.

The arrangement described above was, however, not been further developed for a long time because of difficulties with production of numerous polished square cells of very small size (about $1 \times 1 \mathrm{~mm}$ or smaller at lengths of order of tens of $\mathrm{mm})$.

The early feasibility studies have shown that this demand can be also solved by electroformed replication and first test cells as well as objective prototypes have been already successfully developed in this way. The recent approach is based on electroforming and composite material technology to produce identical triangular segments with square cells while these segments are aligned in quadrants onto a sphere.

The first Angel-developed telescope prototype consists of linear arrangement of 47 square cells of $2.5 \times 2.5 \mathrm{~mm}$,
$120 \mathrm{~mm}$ long (i.e., length/size ratio of almost 50), with focal length of $1.3 \mathrm{~m}$ (Figures 38, 46(a), and 46(b)). The second Angel telescope prototype is represented by an array of $6 \times 6$ that is, 36 square cells, $2.5 \times 2.5 \mathrm{~mm}$ each, $120 \mathrm{~mm}$ long, focus and length/size ratio as above. Both of these prototype modules have been produced and tested already. The microroughness of the inner reflecting surfaces is better than $1 \mathrm{~nm}$. The third prototype was also finished, and consists of $2 \times 18$ perpendicular arranged cells $2.5 \times 2.5 \mathrm{~mm}$, $120 \mathrm{~mm}$ long (Figure 40(a)).

An innovative technique of production of $120 \times 120 \mathrm{~mm}$ sized modules consisting of large number of $3 \times 3 \mathrm{~mm}$ cells, $120 \mathrm{~mm}$ long, is also under development (for related possible arrangement, see Figure 39). 


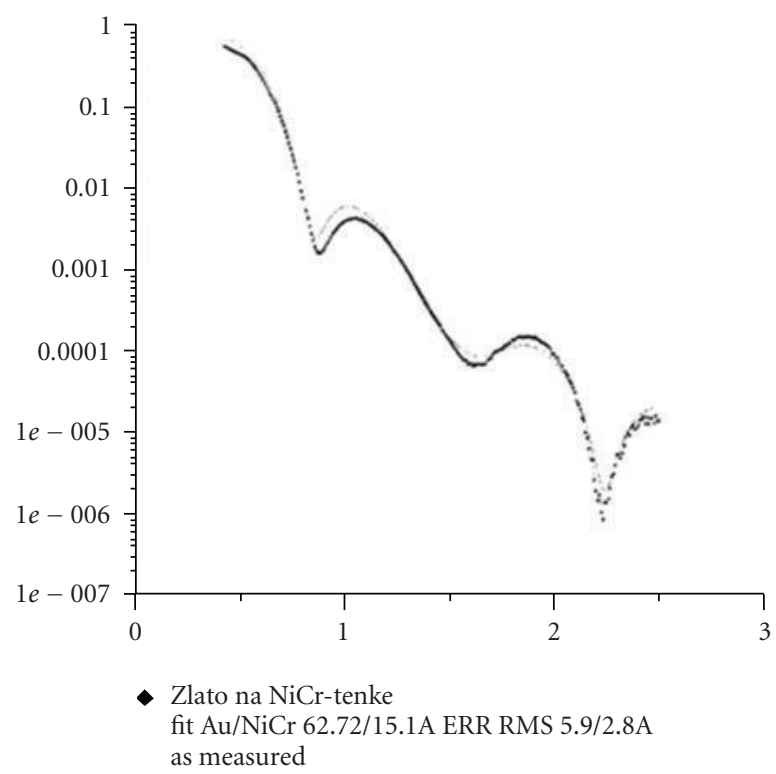

(a)

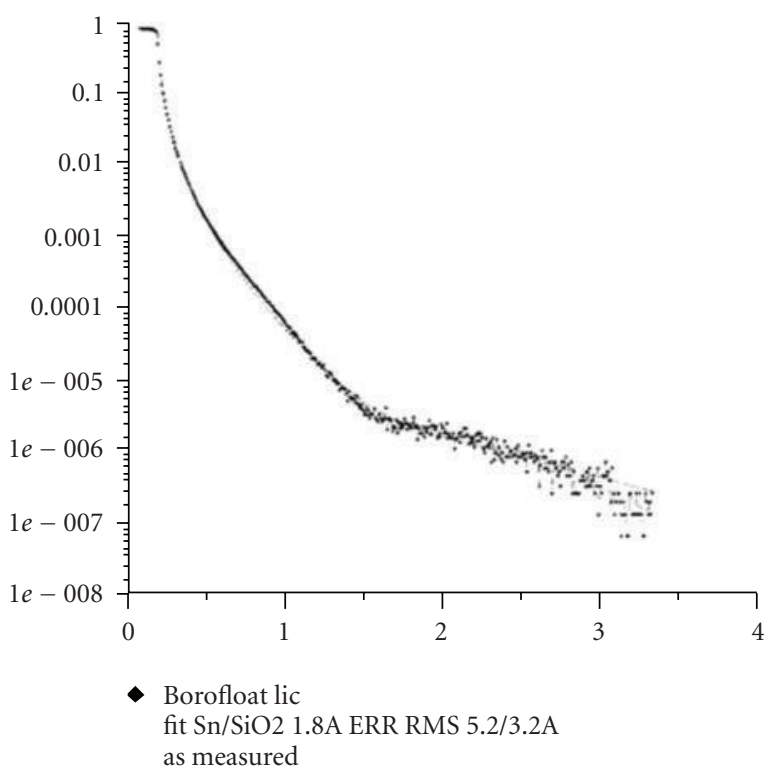

(b)

Figure 31: (a) The X-ray reflectivity measurements of $0.1 \mathrm{~mm}$ float glass plate (used in the $23 \times 23 \mathrm{~mm} \mathrm{Schmidt} \mathrm{objective).} \mathrm{Au/NiCr} \mathrm{layers}$ coated the reflecting surfaces. (b) The X-ray reflectivity measurements for BOROFLOAT $1.1 \mathrm{~mm}$ thick flat sample (with no additional layers).

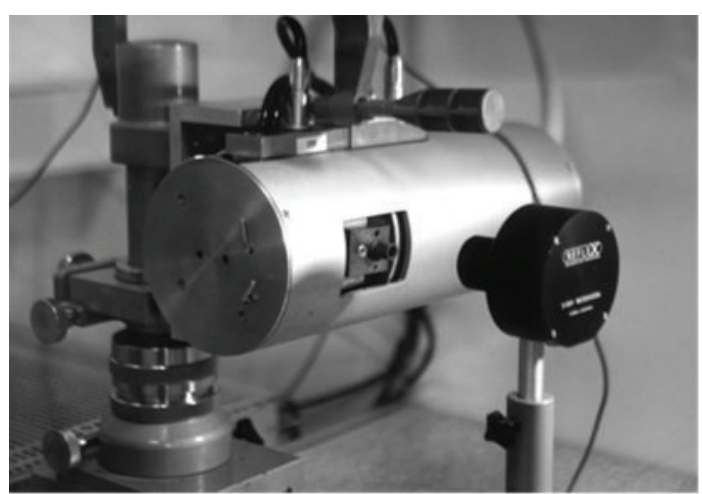

(a)

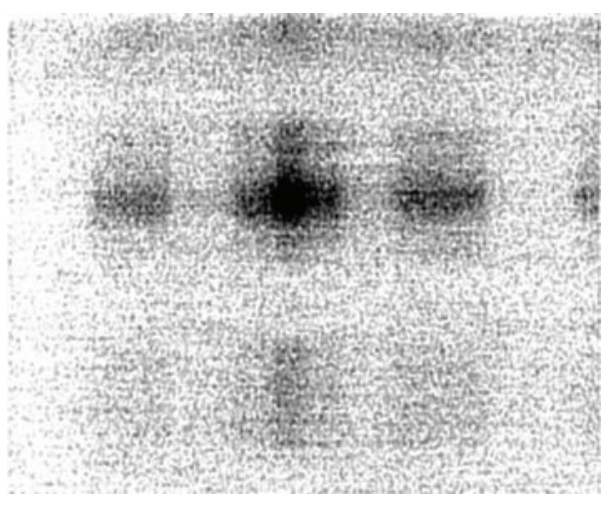

(b)

Figure 32: The Micro source X-ray test facility at Rigaku, Prague (a) and the X-ray image of a 10 micron source at 8 keV detected by X-ray CCD camera (b) in the focal plane of the $3 \times 3 \mathrm{~mm}$ micro Schmidt objective, the image area is $2.5 \times 2.5 \mathrm{~mm}$.

TABle 4: Parameters of the LE Schmidt lenses designed and developed in the Czech Republic.

\begin{tabular}{|c|c|c|c|c|c|c|c|c|c|}
\hline Modul & $\begin{array}{l}\text { Size } d \\
(\mathrm{~mm})\end{array}$ & $\begin{array}{l}\text { Plate thickness } t \\
(\mathrm{~mm})\end{array}$ & $\begin{array}{l}\text { Distance } a \\
(\mathrm{~mm})\end{array}$ & $\begin{array}{l}\text { Length } l \\
(\mathrm{~mm})\end{array}$ & $\begin{array}{c}\text { Eff. angle } \\
a / l\end{array}$ & $\begin{array}{l}\text { Focal length } f \\
(\mathrm{~mm})\end{array}$ & $\begin{array}{c}\text { Resolution } r \\
(\operatorname{arcmin})\end{array}$ & $\begin{array}{c}\text { Field of view } \\
\left({ }^{\circ}\right)\end{array}$ & $\begin{array}{c}\text { Energy } \\
(\mathrm{keV})\end{array}$ \\
\hline macro & 300 & 0.75 & 10.80 & 300 & 0.036 & 6000 & 7 & 16 & 3 \\
\hline midle & 80 & 0.3 & 2 & 80 & 0.025 & 400 & 20 & 12 & 2 \\
\hline $\operatorname{mini} 1$ & 24 & 0.1 & 0.3 & 30 & 0.01 & 900 & 2 & 5 & 5 \\
\hline $\operatorname{mini} 2$ & 24 & 0.1 & 0.3 & 30 & 0.01 & 250 & 6 & 5 & 5 \\
\hline micro & 3 & 0.03 & 0.07 & 14 & 0.005 & 80 & 4 & 3 & 10 \\
\hline
\end{tabular}




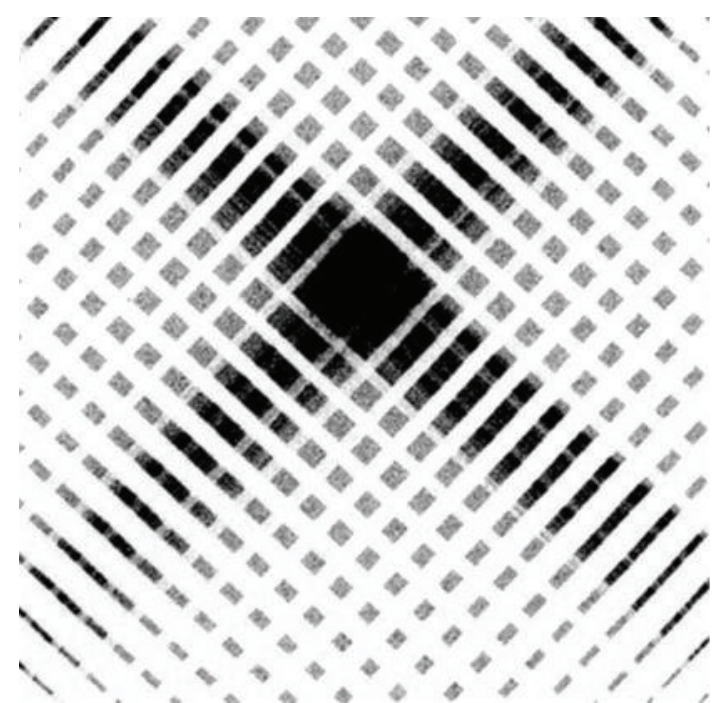

(a)

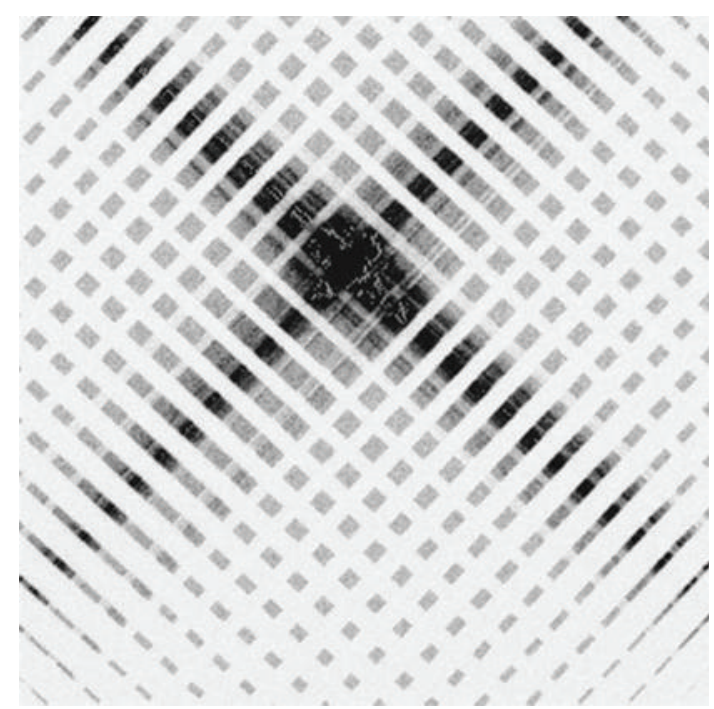

(b)

Figure 33: (a) The focal image of the mini-LE Schmidt (see also Table 4) objective $23 \times 23 \mathrm{~mm}$, at $8 \mathrm{keV}$. X-ray tests were carried out at Rigaku, Prague. (b) The mini-LE Schmidt module X-ray focal spot image, another detector position angle.

4.4. Materials for Lobster Eye Lenses. In general, there is growing need for large segmented X-ray foil telescopes of various geometry and geometrical arrangements. This includes the large modules of the Wolter 1 geometry (e.g., assumed for the future ESA/NASA/JAXA X-ray astronomy mission IXO), the large Kirkpatrick-Baez (further referred as $\mathrm{KB}$ ) modules (as they can play an important role in future $\mathrm{X}$ ray astronomy projects as a promising and less laborious to produce alternative) as well as the large lobster eye modules in the Schmidt arrangements. Although these particular $\mathrm{X}$-ray optics modules differ in the geometry of foils/shells arrangements, they do not differ much from the point of the view of the foils/shells production and assembly, and also share all the problems of calculations, design, development, weight constraints, manufacture, assembling, testing, and so forth. It is evident that these problems are common and rather important for majority of the large aperture X-ray astronomy space projects. All the space projects require light material alternative (e.g., Hudec et al. 2001 [45]).

We have developed the various prototypes of the abovementioned X-ray optics modules based on high quality Xray reflecting gold coated float glass foils (Figure 41, Hudec et al., 2000, [45]). Alternative substrates were also tested (Gorenstein et al. 1996 [7]). The glass represents a promising alternative to widely used electroformed nickel shells, the main advantage is much lower specific weight (typically $2.2 \mathrm{gcm}^{-3}$ if compared with $8.8 \mathrm{gcm}^{-3}$ for nickel). However, the technology needs to be further exploited and improved in order to achieve the required accuracy. For the large prototype modules of dimensions equal or exceeding $30 \times$ $30 \times 30 \mathrm{~cm}$, mostly glass foils of thickness of $0.75 \mathrm{~mm}$ have been used for these large modules, although in future this thickness can be further reduced down to $0.3 \mathrm{~mm}$ and perhaps even less (we have successfully designed, developed and tested systems based on glass foils as thin as 30 microns, albeit for much smaller sizes of the modules, see Figure 45).

The requirement of minimizing the weight of future large $\mathrm{X}$-ray space telescopes and at the same time achieving huge collecting areas means that the future large astronomical mirrors have to be based on thin X-ray reflecting foils that is, thin layers with low weight which can be easily multiply nested to form the precise high throughput systems (Figures 44(a), and 44(b)). Below, we discuss some of the analyzed techniques and approaches and related progress.

\subsection{The Application and the Future of Lobster-Eye Telescopes.} It is obvious that the first lobster-eye prototypes confirm the feasibility to design these telescopes with currently available innovative technologies (Figure 47). We propose the following steps to be undertaken for a real wide-field $\mathrm{X}$-ray telescope: (1) to reduce further the microroughness as well as the slope errors of the reflecting surfaces in order to improve the angular resolution and the system reflectivity/efficiency. The recent development has already lead to significant microroughness improvement (to $0.2-$ $0.5 \mathrm{~nm}),(2)$ to design and to construct larger or multiple modules in order to achieve larger fields of view (of order of 1000 square degrees and/or more) and enhanced collecting area, (3) to reduce further the aperture of the cells (for the Angel arrangement) and/or spacing and plate thickness (Schmidt arrangement) and to enhance the length/aperture ratio (recently nearly 50-80), and (4) to study the multilayer application on reflecting surfaces and/or other approaches in order to improve the energy coverage for higher energies.

The application of very wide field X-ray imaging systems could be without any doubt very valuable in many areas of $\mathrm{X}$-ray and gamma ray astrophysics. Results of analyses and simulations of lobster-eye X-ray telescopes have indicated 

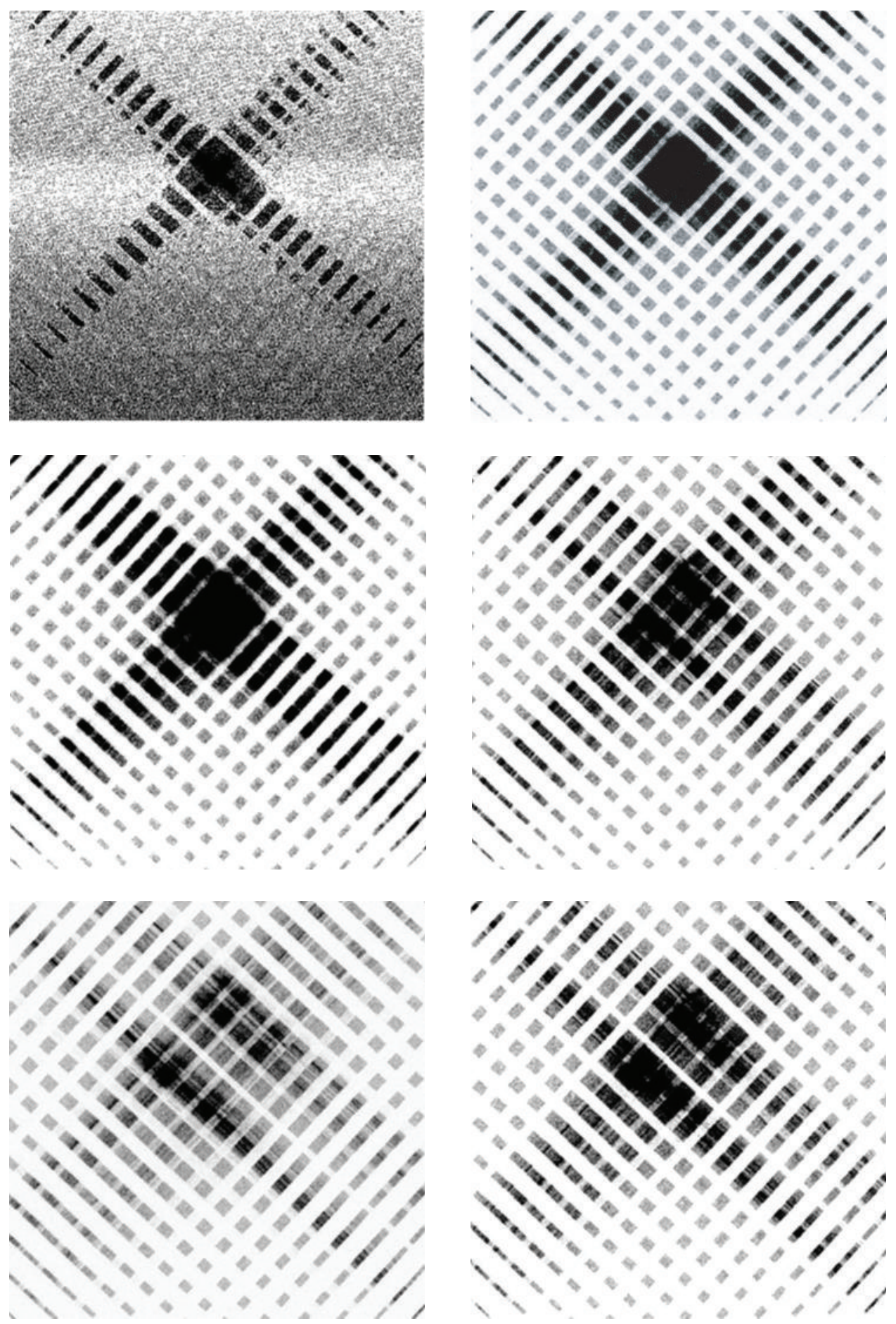

FIGURE 34: A sequence of X-ray focal spot images in front, in focus and behind the focal plane showing the defocusing behaviour of the mini LE Schmidt module (the distance between the detector position for first and the last image is $110 \mathrm{~mm}$ ).

that they would be able to monitor the X-ray sky at an unprecedented level of sensitivity, an order of magnitude better than any previous X-ray all-sky monitor. Limits as faint as $10^{-12} \mathrm{erg} \mathrm{cm}^{-2} \mathrm{~s}^{-1}$ for daily observation in soft X-ray range are expected to be achieved, allowing monitoring of all classes of X-ray sources, not only X-ray binaries, but also fainter classes such as AGNs, coronal sources, cataclysmic variables, as well as fast X-ray transients including gammaray bursts and the nearby Type II supernovae. For pointed observations, limits better than $10^{-14} \mathrm{erg} \mathrm{sec}^{-1} \mathrm{~cm}^{-2}(0.5$ to $3 \mathrm{keV}$ ) could be obtained, sufficient enough to detect Xray afterglows to GRBs. As indicated by our preliminary results, the production of corresponding optical elements can be reasonably achieved by electroforming methods and 


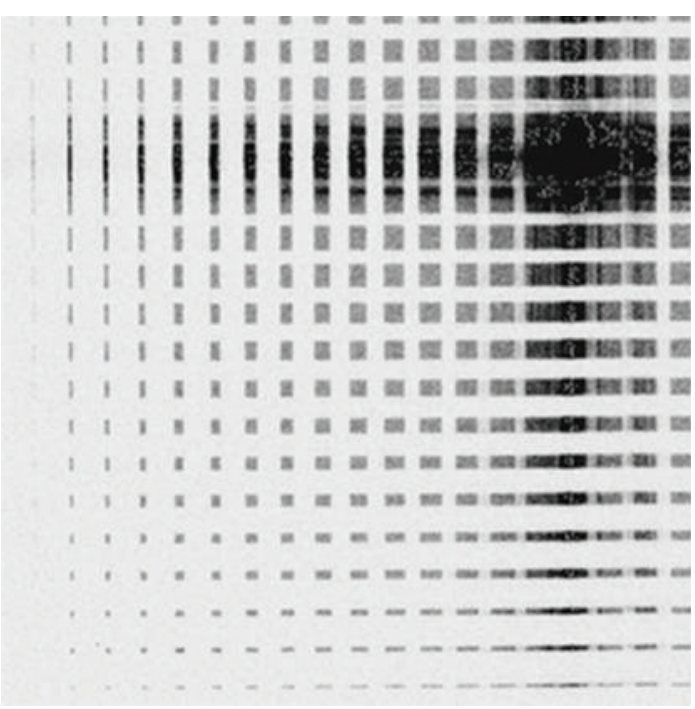

(a)

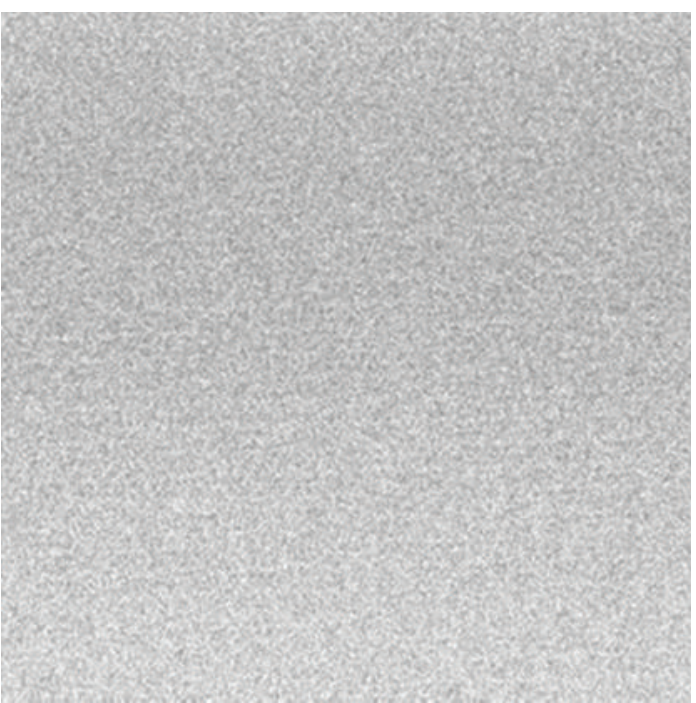

(b)

FIgURE 35: The two X-ray images (signal exposure and dark-background image) exhibiting the focal spot of the LES module as well as the detection capability of the used X-ray CCD 16 bit detector. The average dark signal is $266 \mathrm{ADU}$ at the exposure of 80 sec ADU, the rms noise is $2 \mathrm{ADU}$, that is, the achieved electronic dynamic range is more than 30000 . The measured intensity gain of this module is 420 .

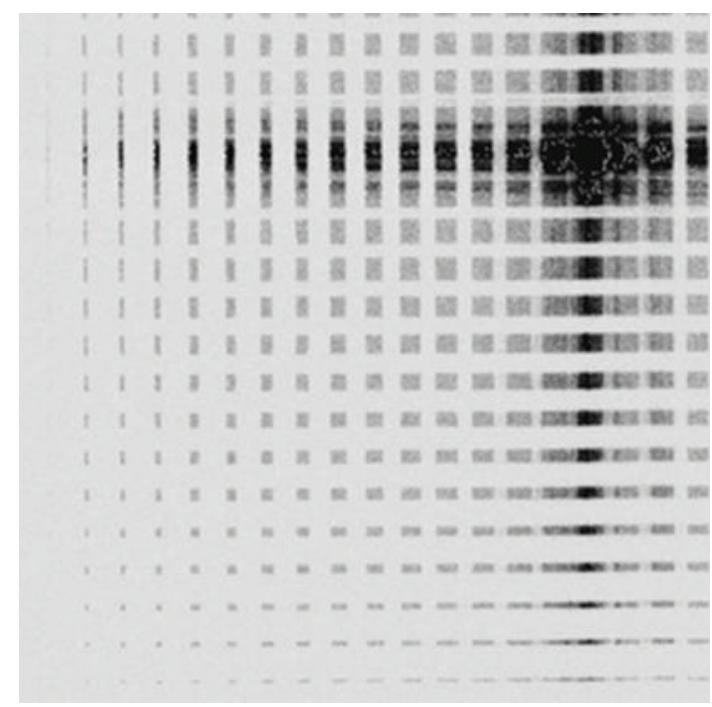

(a)

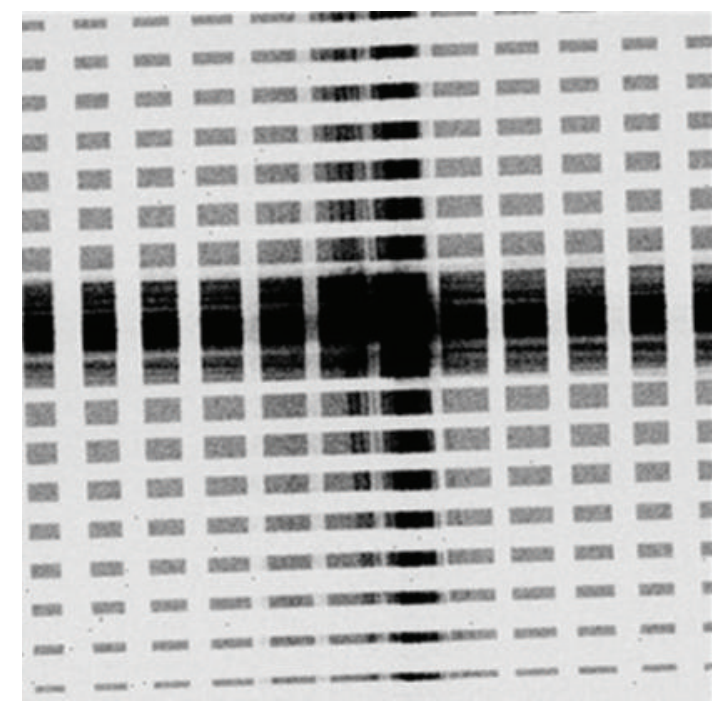

(b)

Figure 36: (a) The X-ray focal image of the MINI LE SCHMIDT module showing the main intensity to be inside the main focal spot. The intensity gain achieved is 570 (for $8 \mathrm{keV}$ X-ray tube and only part of the LE module active due to the high energy of X-ray photons-note that this module has been designed for energy of $2 \mathrm{keV}$ ). (b) The focal plane image for the LE test module. The measured gain is 500.

composite replication as the alternative to other methods. For the Schmidt objectives, the results obtained with the development of technology for production of large area and high-quality double-sided X-ray foils are very promising and together with composite material technologies represent an important input for the further development of this type of X-ray optics. The production of Angel lobster-eye cells is much more complicated, nevertheless the first prototypes of the lobster eye Angel cells have been also successfully designed and developed.
4.6. LE Laboratory Modifications. The lobster eye soft X-ray optics, originally proposed and designed for astronomical (space) applications, have potential for numerous laboratory applications.

As an example, LE optics can be modified for efficient collection of laser-plasma radiation for wavelengths longer than $8 \mathrm{~nm}$ (Bartnik et al., 2006 [55]). The optics for this application consists of two orthogonal stacks of ellipsoidal mirrors forming a double-focusing device (Bartnik et al., 2006 [55]). The ellipsoidal surfaces were covered by a layer 


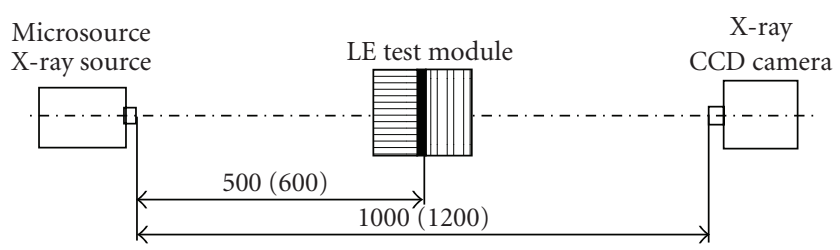

FIgURE 37: The arrangement of the X-ray tests of the LE modules.

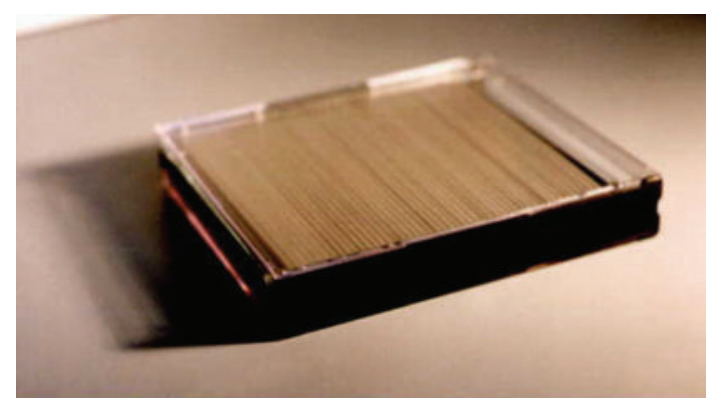

Figure 38: The unique linear Angel prototype produced by replication: 47 square channels, $120 \mathrm{~mm}$ long, and $2.5 \times 2.5 \mathrm{~mm}$. This picture illustrates the power of electroformed replication even for very long square cells which otherwise could not be polished inside. In this technology, the square mandrels were highly polished and then replicated, so the polished surface is now inside. This demonstrates that also other techniques as MCP can be considered for LE Angel arrangements.

of gold that has relatively high reflectivity at the wavelength range between $8-20 \mathrm{~nm}$ up to about 10 degrees of an incidence angle. A schematic view of half of the optics system together with a front view of the optic, are shown, respectively, in Figure 48. The mirrors forming the optics have the width of $40 \mathrm{~mm}$. As can be noticed the spacing between adjacent mirrors rises with the distance from the axis. The curvature of the mirrors and the spacing between them were optimized by ray-tracing simulations to maximize an aperture of the optic and minimize the size of the focal spot.

The distances for the best focusing were found as $181 \mathrm{~mm}$ source-LE entrance, $183 \mathrm{~mm}$ LE rear cover-focus. The relative intensity distribution of the visible light at the focal spot is presented at Figure 48.

4.7. Hybrid LE. The lobster eye optics described in the previous section are wide field optics, but it is relatively complicated to achieve a better angular resolution with the given technological limitations of mirror thickness and spacing (Sveda et al. [56]).

One of possible solutions to this problem is induced by the typical use case of the standard lobster eye as an All Sky Monitor (ASM) for X-ray astronomy. The lobster eye will be used onboard the satellite and will scan the sky. If a certain point of the sky is outside the FOV of the optics at the moment, it will be inside the FOV sometime later because of scanning. Hence, in principle, we can accept smaller FOV in the scanning direction, if we can get some advantage as a trade-off, such as a better angular resolution. The desired optics would have a wide FOV and moderate angular resolution in one direction, and a smaller FOV and better angular resolution in another one.

It is necessary to use curved mirrors to achieve the better angular resolution with constraints on mirror dimensions. There is a relatively simple way to the final idea combining all these facts. A combination of the standard one-dimensional lobster eye in one direction and the Kirkpatrick-Baez parabolic mirror set in the other direction would fulfil all the requirements (Sveda et al., 2005 [56]), see Figure 49.

The preliminary results show that the hybrid lobster Eye can work as intended, that is, it increases the angular resolution in one direction while still having wide FOV in another. However, the blurring increases rapidly with the offaxis distance in the direction where focusing the parabolic mirrors are. Consequently, it is reasonable to think about such optics for point observations if the source and/or image are expected to be highly asymmetric. The effect of blurring is reduced for scanning observations, hence the increase in angular resolution is well achievable, but the decrease of gathered photons resulting in much worse limiting flux is so significant that, together with manufacturing difficulties, this optics is probably not usable for X-ray astronomy but may find usage in laboratory.

4.8. MCP as Alternative LE Optics. Alternative grazing incidence X-ray optics can use microchannel plate-MCP, in a Wolter-like or alternatively lobster-eye type, X-ray optic (for a detailed review, see, e.g., [57]). Depending on the application (laboratory or space) the MCP can be either flat or curved.

The MCP, when it is curved into a spherical geometry and square profile channels are used, becomes equivalent to the so-called lobster-eye telescope first proposed by Angel (1979 [60]) and is closely related to the orthogonal mirror proposed by Schmidt (1975 [53]). Chapman et al. [102] published an exhaustive theoretical treatment of the properties of square channel arrays (Figure 50) and detailed investigations and proposals based on application of MCP as LE lenses were reported, for example, by Priedhorsky et al. (1996 [62]), Fraser (1993 [71]) and Peele (2001 [103]), see also Figure 51.

The X-ray LE optic systems in Angel arrangement are based on slumped lead glass microchannel plates (MCPs). However, the spectral range of operation of existing MCPbased LE optics is limited to X-rays with energies of less than $4 \mathrm{keV}$. Harder X-rays with smaller critical grazing angles cannot be focused efficiently by glass MCPs because their walls are inaccessible for polishing and metallization. This energy range constraint of the MCP-based LE optics limits its application to vacuum chambers or open-space astronomical applications with infinite distance to the objects (such as celestial objects).

To reach their full capabilities, MCPs must be manufactured accurately to reduce figure error and have low surface roughness to reduce scattering. Their being manufactured from or coated in a material with high X-ray reflectivity is 


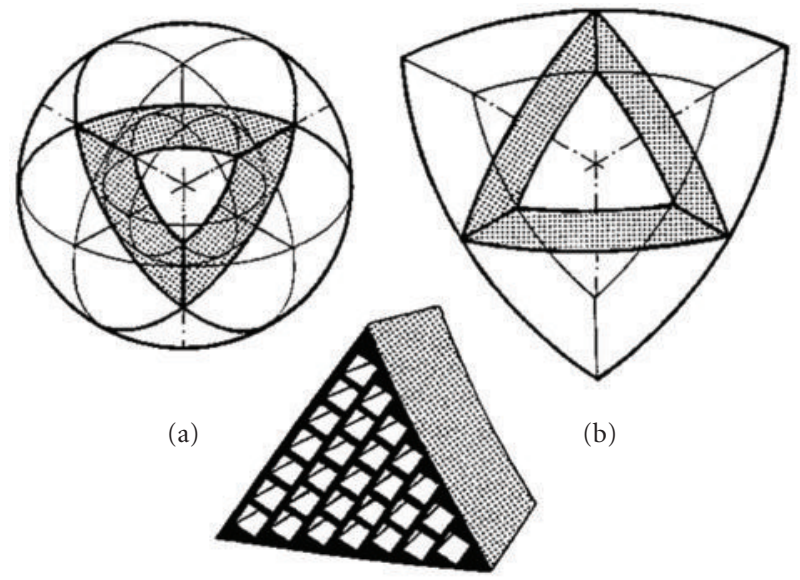

(c)

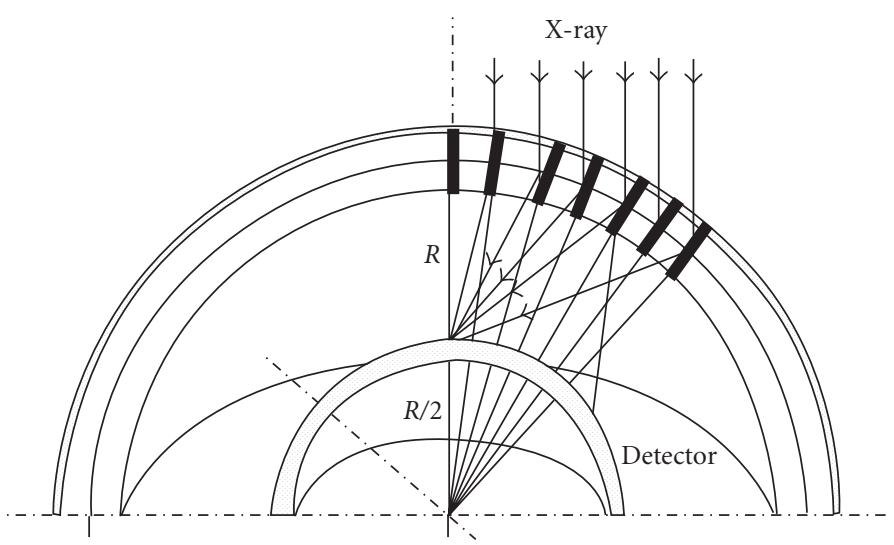

FIGURE 39: The schematic arrangement of the Angel lobster-eye objective (bottom) and the proposed construction based of electroformed triangular segments of this arrangement (top). The triangular segments represents parts of the sphere (a, b) and include square cells of Angel LE arrangement (c).

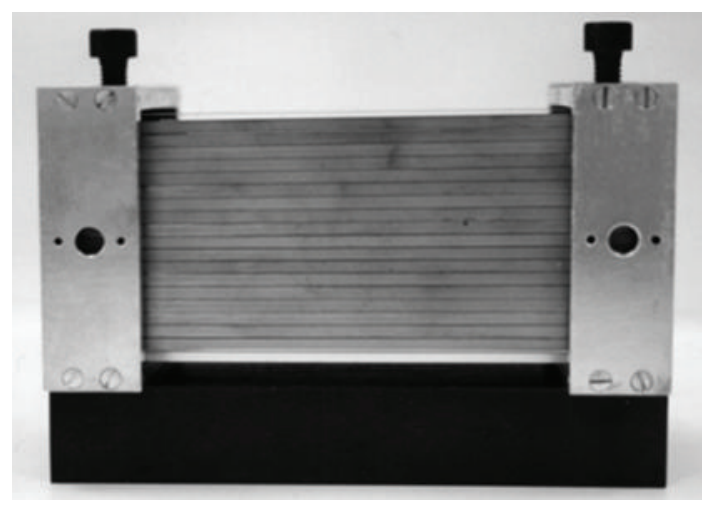

(a)

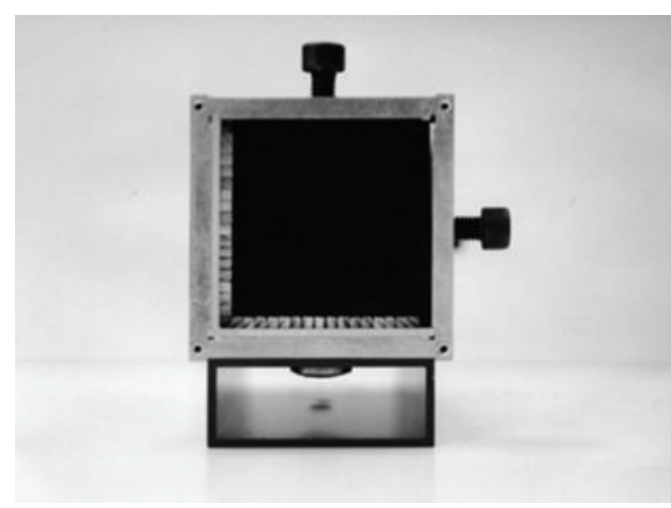

(b)

FIGURE 40: The Angel lobster eye test module, in L-shaped array of $2 \times 18$ cells, $2.5 \times 2.5 \mathrm{~mm}, 120 \mathrm{~mm}$ long.

also desirable. Initial investigations of MCPs have used flat samples because figure error and surface roughness can be readily evaluated and samples are easier to prepare (Figure 52).

The cruciform structure of the focal spot for square profile channels was observed in the X-ray region for example, by Fraser et al. [71], though the focal spot itself was somewhat broader than it was expected because of imperfections in the MCP (Figure 53).

The focusing performance of the MCP may be effectively understood with the theoretical results published by Chapman et al. [102]. The MCP consists of an array of square 


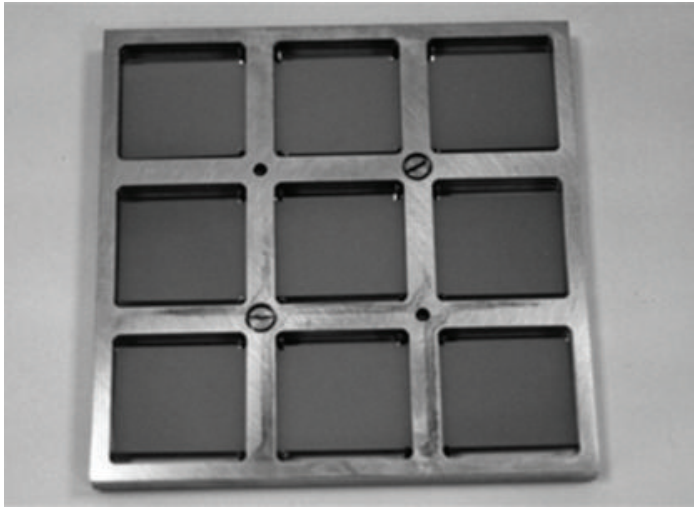

(a)

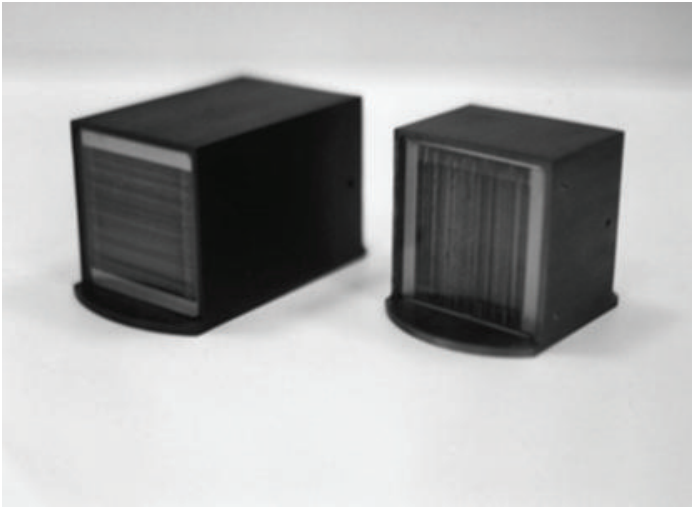

(b)

FIGURE 41: The frame with $23 \times 23 \mathrm{~mm}$ flats for the Schmidt objective prototype after gold coating (a) and the assembled modules (b).

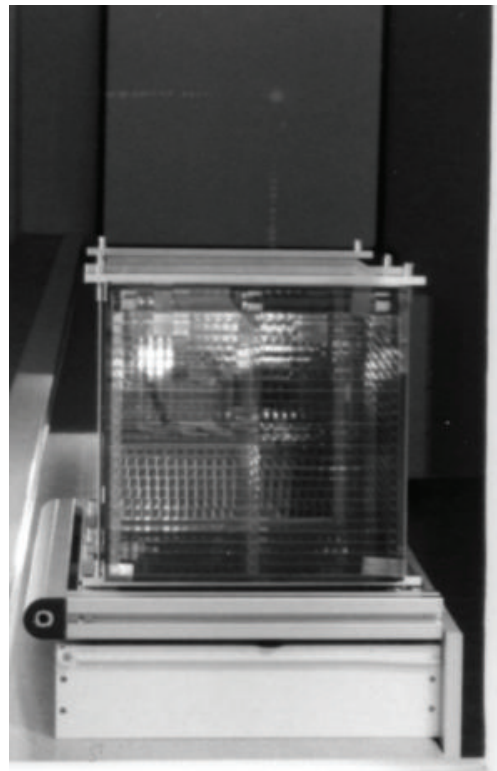

Figure 42: The large $(30 \times 30 \mathrm{~cm})$ LE Schmidt lens during optical tests (with optical focal image).

channels. $\mathrm{X}$ rays that strike the exterior walls on the face of the MCP are lost. Of the X-ray photons that enter a channel, a fraction that reflect once off two orthogonal walls are reflected into the focal spot, another fraction are reflected only from one wall and so are focused in one dimension to a line passing through the two-dimensional focus, and a third fraction pass straight through the array to form an unfocused background. Higher-order reflections are also possible, but the X-ray photons from such reflections strike a detector as if they were in one of the above mentioned classes. The resulting focal structure then consists of a bright focused spot with a fainter cross centred on this and a much less intense diffuse background. The relative number of photons in each of the above described structures depends on the ratio of the width of the channels to their length. At the optimal ratio for a lens with no axis of symmetry and

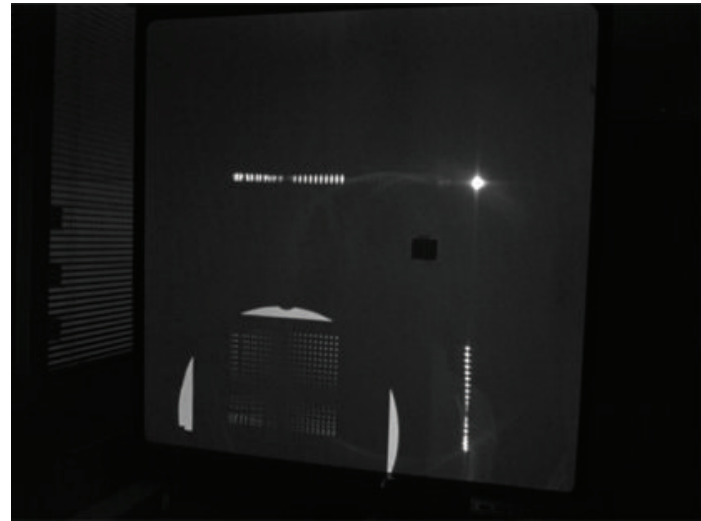

FIGURE 43: The focal plane image from the large $(30 \times 30 \mathrm{~cm}) \mathrm{LE}$ 2D module ( $0.75 \mathrm{~mm}$ thick glass plates, optical light).

with $100 \%$ reflectivity, $34.3 \%$ of the photons are focused into the central square, $24.3 \%$ end up in each of the one dimensional foci arms, and $17.2 \%$ end up in the unfocused background. The focusing efficiency in a given direction may be improved when a preferred axis is imposed on the device, but this is not consistent with a very-wide-fieldof-view telescope. The $\mathrm{X}$ rays are brought to a focus with an angular resolution comparable with the angle that an individual channel subtends at the detector. In practice this implies that the MCP based lobster-eye telescope will always be limited in resolution by the physical size of the individual channels.

\subsection{Comparison of MFO and MCP Design of LE Optics.} Direct and reliable comparison of MFO and MCP designs of LE X-ray optics is difficult, as in both cases, the real optics performance deviates from theoretical one. For MCP design, the necessary slumping obviously introduces additional sources of errors (Bannister et al., 2007 [75]).

Some of the examples of the performance are shown below together with Table 5 comparing both approaches. 


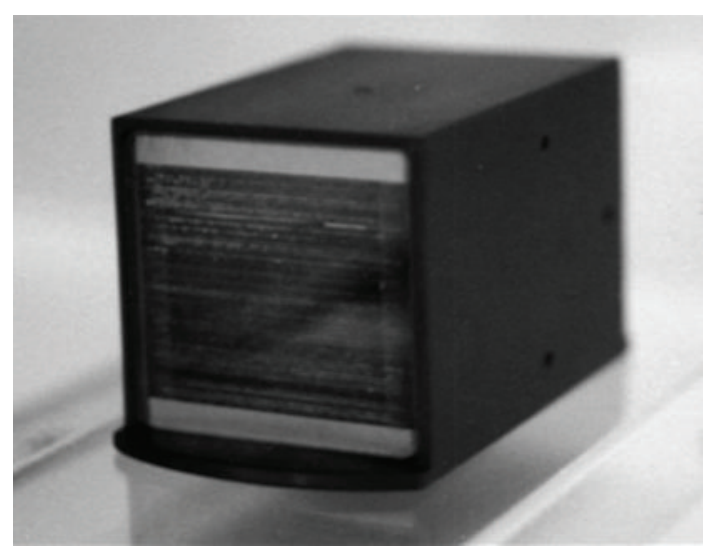

(a)

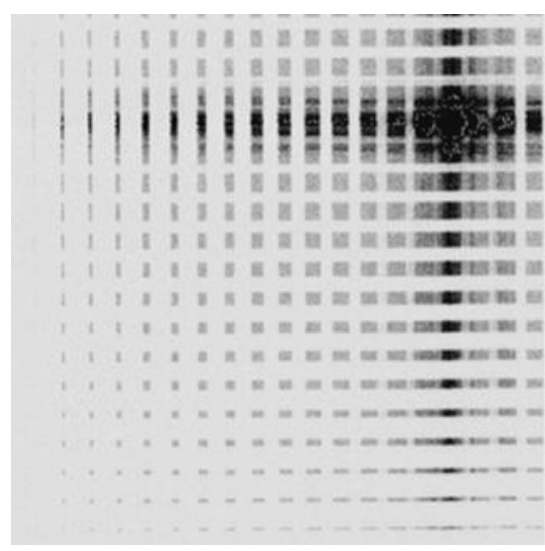

(b)

FIgURE 44: (a) The mini $(24 \times 24 \mathrm{~mm}, 0.1 \mathrm{~mm}$ thick foils spaced at $0.3 \mathrm{~mm})$ Schmidt LE module illuminated by the laser beam. (b) The mini Schmidt 2D prototype X-ray $(8 \mathrm{keV})$ focal spot image (image area $12.3 \times 12.3 \mathrm{~mm}$ ). The measured gain was 680 .

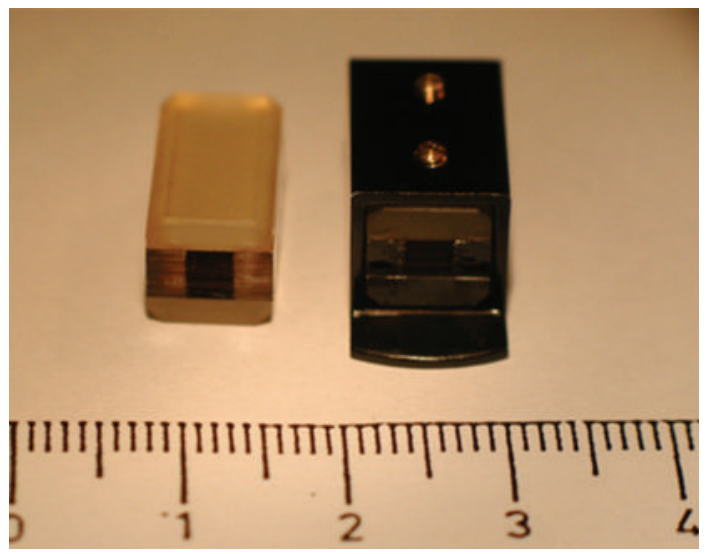

Figure 45: The micro LE Schmidt objectives, $3 \times 3 \mathrm{~mm}$. This figure illustrates that the MFO technology can be applied also for very small structures and very small Lobster Eye modules. In the devices illustrated, gold-coated float glass foils 30 micron thick are assembled with spacing of 70 microns. Small plate distances (together with long focal distance) represent condition for good angular resolution.

Examples of recent measurement results for MFO LE design (Figures 54, 55, and 56) and MCP LE design (Figure 57) is shown below. For more details, see the given references.

\section{Capillaries}

Although the capillaries represent collimating and not imaging elements, we note them here for completeness. Condensing an X-ray beam by using total external reflection along the interior surface of a hollow tapered glass capillary has been demonstrated by Stern et al. [104]. Tapered glass monocapillaries have been fabricated by many investigators (e.g., Engstrom et al., 1991 [105]). These capillaries condense $\mathrm{X}$-ray beams by the use of geometrical reflection optics alone. $\mathrm{X}$ rays incident at glancing angles to the interior surface of the tapered capillary are reflected along its length toward an exit

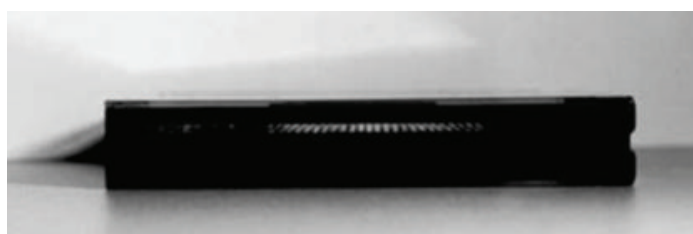

(a)

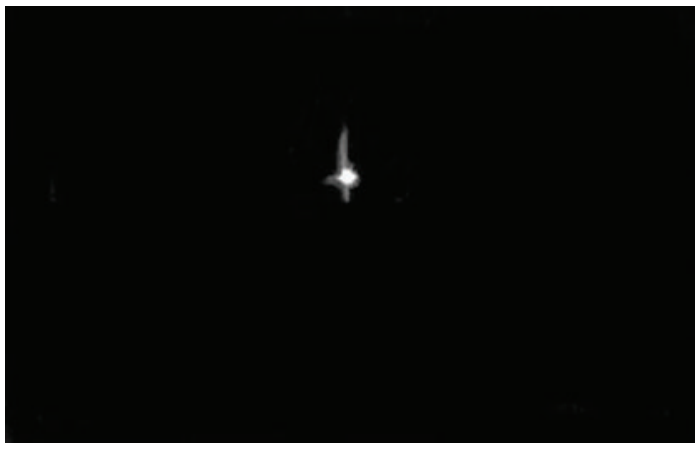

(b)

FIgURE 46: (a) The Angel LE linear prototype (47 cells $2.5 \times 2.5 \mathrm{~mm}$, $120 \mathrm{~mm}$ long). (b) The optical focal image from the linear LE prototype in Angel arrangement.

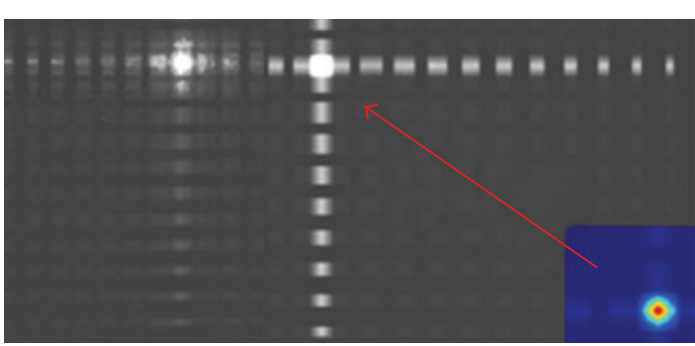

FIGURE 47: Experiment (left) and simulation (right) of a point-topoint focusing LE MFO test optic. $8 \mathrm{keV}$ photons are used.

aperture of smaller dimensions than its entrance aperture. The decrease in the relative area of the apertures corresponds to an increase in flux per unit area (intensity) in the emerging 


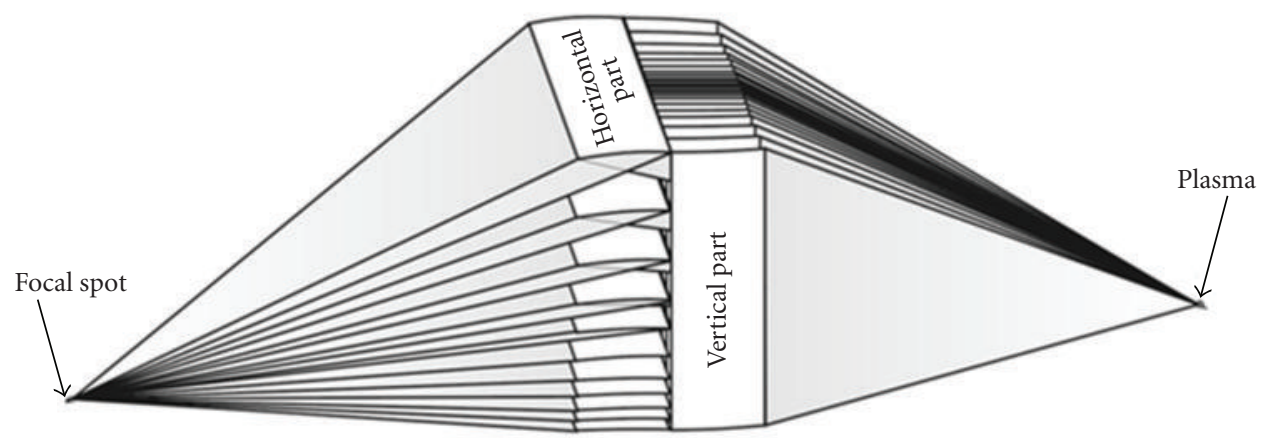

(a)

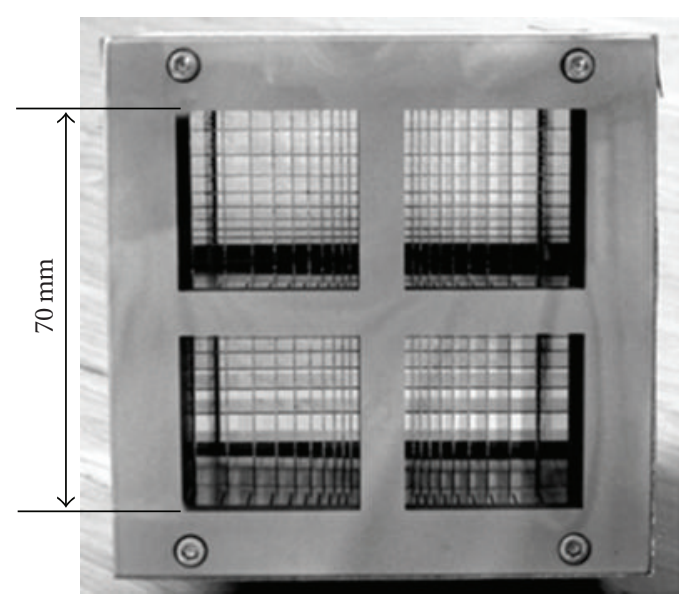

(b)

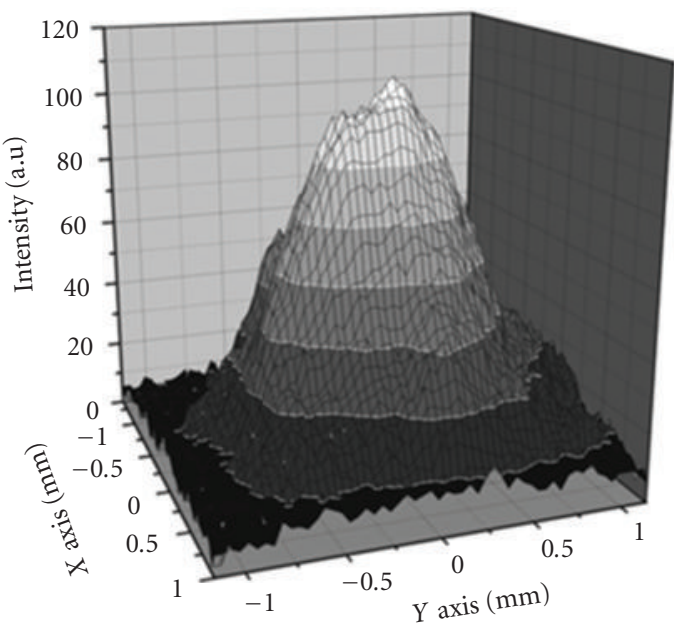

(c)

FIgURE 48: The lobster eye optic: (a) schematic view of half of the optic system, (b) front view of the optic (c) simulation of the intensity distribution at the lobster eye focus using $0.3 \mathrm{~mm}$ visible light source (Bartnik et al., 2006 [55]).

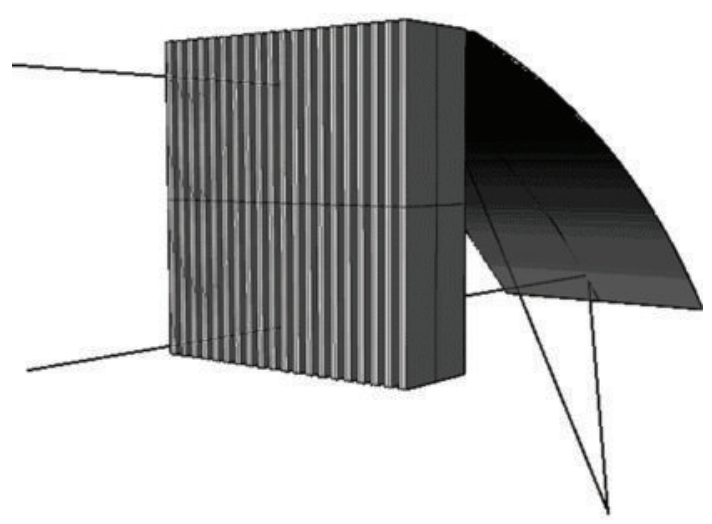

Figure 49: The sketch of the Hybrid Lobster Eye with two plotted rays. Only one parabolic mirror is schematically plotted here. In fact, a number of reflecting surfaces have to be used (Sveda et al., $2005[56])$.

beam. The reflection of $\mathrm{X}$ rays from the interior surface of the capillary is achieved through total external reflection from the glass surface. This specular reflection process occurs because the refractive index of the glass for $\mathrm{X}$ rays is less

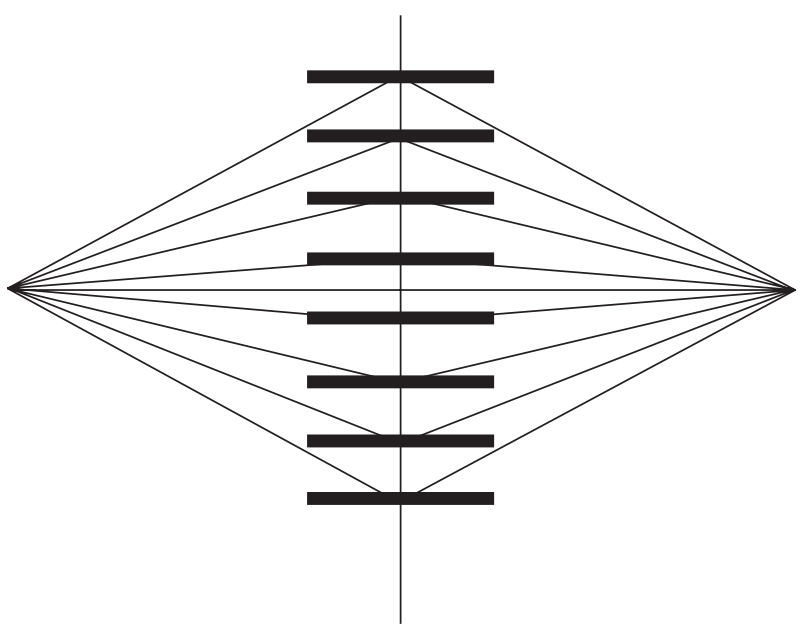

FIGURE 50: Focusing from a point source using a multichannel plate optic (MacDonald and Gibson, 2010 [57]).

than unity. For an ideally flat surface one may characterize this reflectivity using the Fresnel reflectivity function for a given glass composition. For X rays with an energy of $6 \mathrm{keV}$ or more, the reflectivity is often well described as a 


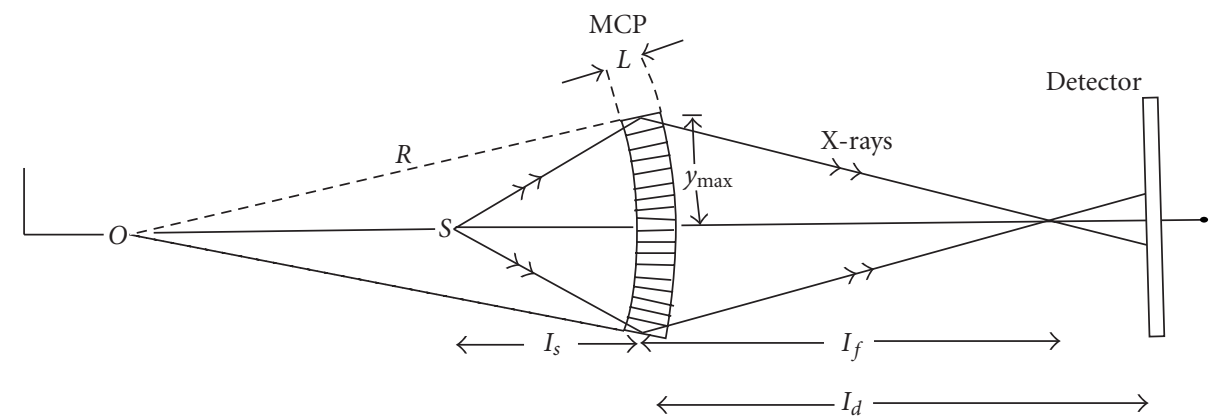

FIGURE 51: Creating a collimated beam with a slumped multichannel plate (Fraser et al. 1993 [71]).
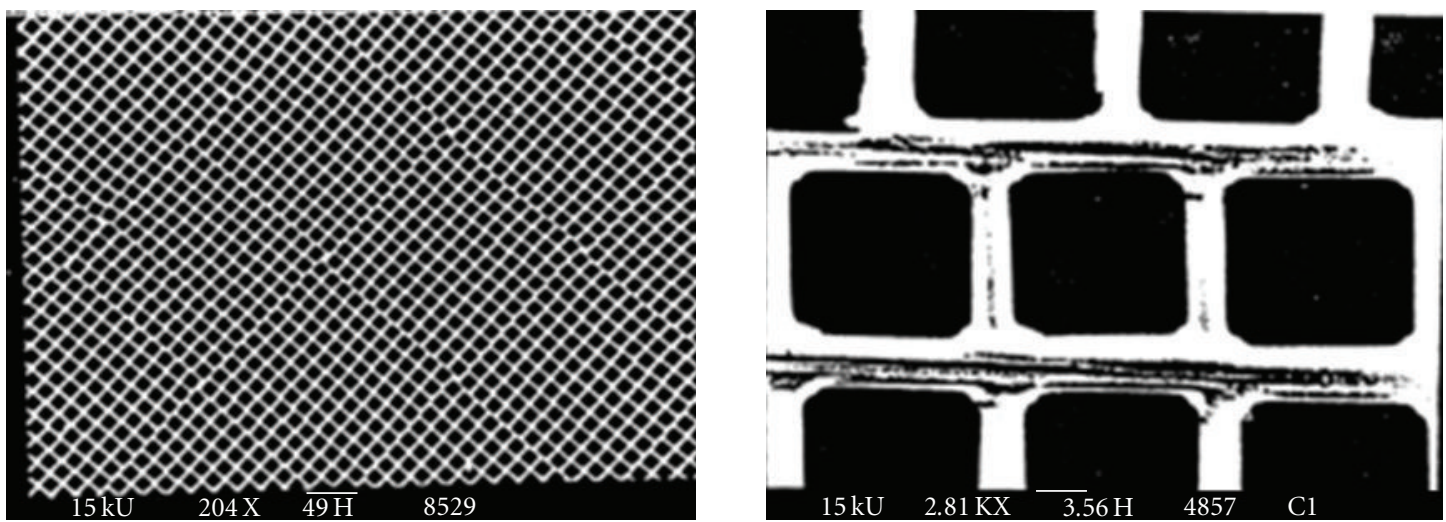

FIGURE 52: Square pore multichannel plate with 13.5-micron pore size, magnified at right (Brunton et al., 1995 [72]).

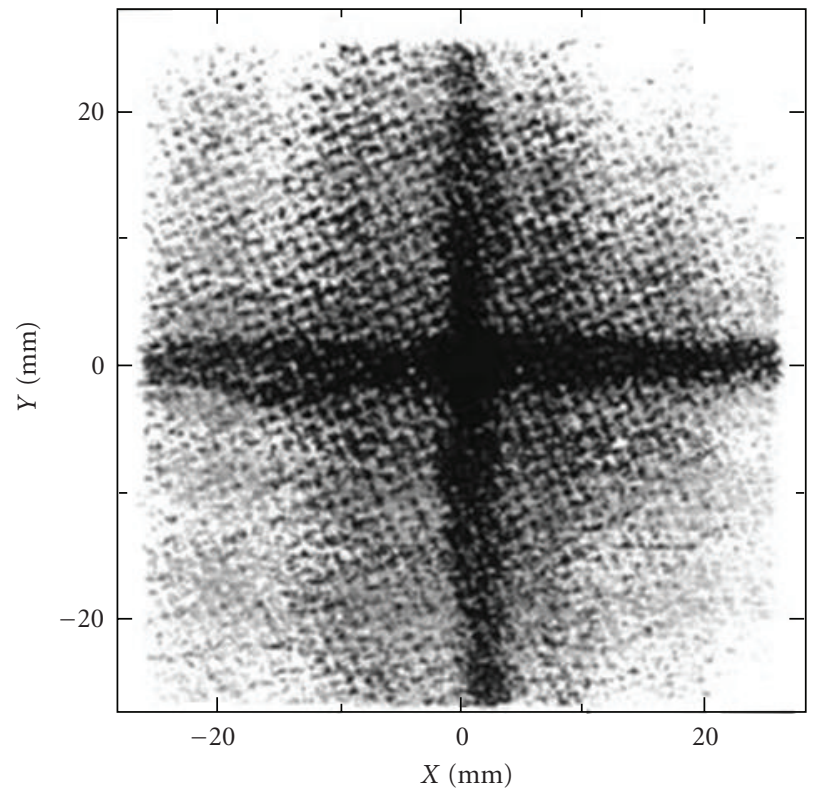

(a)

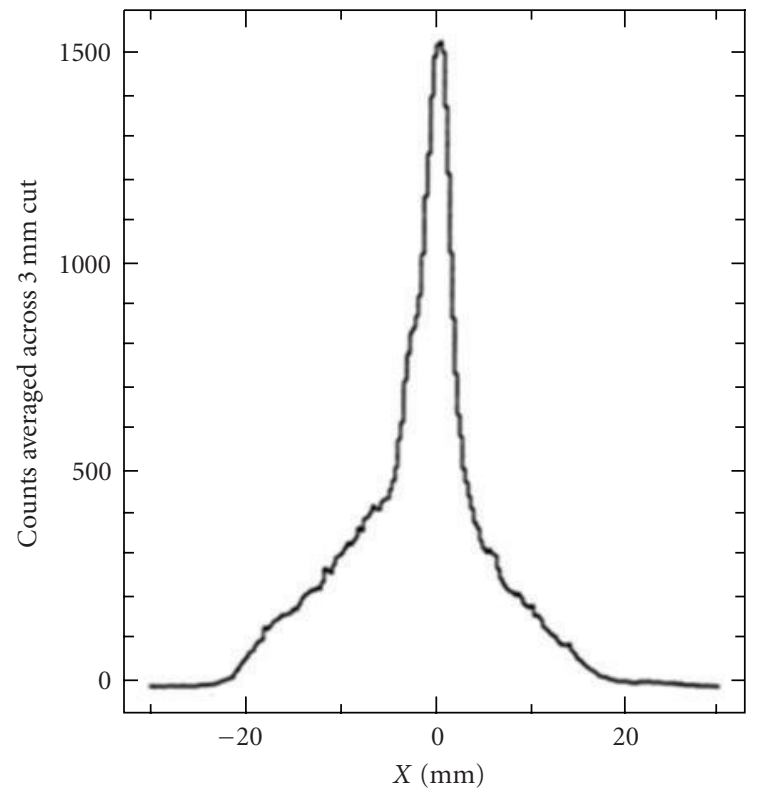

(b)

FIGURE 53: (a) Output image from MCP with $11 \mu \mathrm{m}$ square pores and thickness to diameter ratio of $40: 1$ illuminated with $1.74 \mathrm{keV}$ Si K X rays. (b) Scan of image with the same MCP, taken with $0.28 \mathrm{keV} \mathrm{C} \mathrm{K} \mathrm{X} \mathrm{rays} \mathrm{(Brunton} \mathrm{et} \mathrm{al.,} 1995$ [72]). 
TABLE 5: Multifoil versus MCP approach to the Lobster ASM.

\begin{tabular}{|c|c|c|c|c|}
\hline Property & MCP & & & Multi-foil \\
\hline Surface microroughness & $10-20 \AA[54,55,90]$ & - & + & $3-10 \AA[57,102]$ \\
\hline Metal coating & More complicated, getting better & - & + & Simple, standard \\
\hline Multilayers & Very difficult & - & + & Simple, standard [22] \\
\hline $\operatorname{Gain}^{1}(1 \mathrm{keV})$ & $10-100(30-40$ derived from $[54])$ & - & + & $100-1000(\sim 2500$ simulation $\sim 1000$ reality $)$ \\
\hline Gain $^{2}(8 \mathrm{keV})$ & Not documented & - & + & $10-100$ (300-400 [21], point-to-point focus) \\
\hline Optics Weight ${ }^{3}$ & $\sim 0.1 \mathrm{~kg}[54]$ & + & - & $\sim 1.0 \mathrm{~kg}$ \\
\hline Optics dimensions ${ }^{4}$ & $70.0 \times 70.0 \times 0.3 \mathrm{~mm}[54]$ & + & - & $78 \times 78 \times 23 \mathrm{~mm}$ \\
\hline FOV & Unlimited in principle & + & - & Wide but limited \\
\hline Energy range & $<3 \mathrm{keV}[54]$ & - & + & $<10 \mathrm{keV}^{5}$ \\
\hline ASM module FOV ${ }^{6}$ & $\sim 20 \times 20 \operatorname{deg}[54]$ & + & - & $6 \times 6 \operatorname{deg}$ \\
\hline Daily limiting flux $\left[10^{-13} \mathrm{erg} \mathrm{s}^{-1} \mathrm{~cm}^{-2}\right]$ & $\sim 20[54]$ & - & + & $\sim 5-10^{7}$ \\
\hline Angular resolution & $\sim 4 \operatorname{arcmin}$ & + & + & $\sim 4 \operatorname{arcmin}$ \\
\hline Detector shape & Curved [54] & - & + & Planar \\
\hline
\end{tabular}

${ }^{1}$ Defined as a ratio of photons gathered inside the FWHM with and without optics.

${ }^{2}$ Defined as a ratio of photons gathered inside the FWHM with and without optics.

${ }^{3} 6 \times 6$ deg FOV, $f=37.5 \mathrm{~cm}$, both approaches scaled to fit these criteria.

${ }^{4} 6 \times 6$ deg FOV, $f=37.5 \mathrm{~cm}$, both approaches scaled to fit these criteria.

${ }^{5} \mathrm{Au}$ coated, microroughness $\sigma \sim 10 \AA$.

${ }^{6}$ The rating of this property is disputatious: larger FOV results in smaller number of modules to build and test, on the other hand, if anything goes wrong, larger part of the sky will be uncovered and higher costs for insitu repair is demanded.

${ }^{7}$ Depends on the PSF blurring by distortions, backgroung estimates....
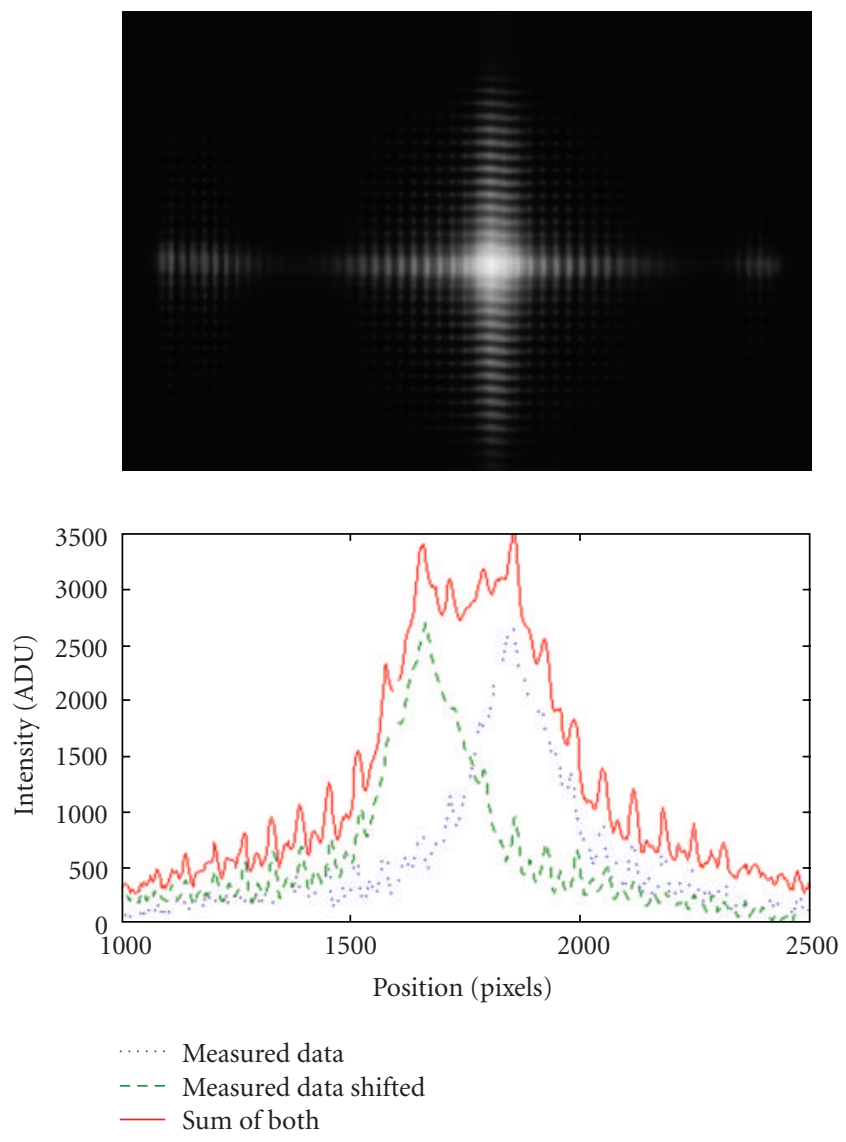

Figure 54: Measurements of MFO LE optics with $f=25 \mathrm{~cm}$ in $8 \mathrm{keV}$ with Medipix 2 detector with measured FWHM of 8 arcmin (Tichy et al., 2010a [73]). step function with respect to the grazing angle of $\mathrm{X}$ rays to the surface, with the position of the step termed the critical angle. Approximately $90 \%$ of the ray's incident below this critical angle reflects specularly from the surface (Balaic and Nugent, 1995 [106]). The electroformed micromirrors represent another alternative to single capillaries (Arndt et al. 1998 [107], Pina et al. 1996 [103], and Pina et al. 2000 [108]).

Several factors conspire to degrade the gain in intensity expected in the emerging beam from the capillary. These include poor interior-surface quality of the glass (roughness), the absorption of $\mathrm{X}$ rays by the ambient gases present within the capillary, and limitations in the capacity of the capillary to accept the range of incident $\mathrm{X}$-ray angles available from the source (Balaic and Nugent, 1995 [106]). The latter constraint is determined by the angular limits of total external reflection for the type of glass and X-ray energies used.

Systems involving the use of a large number of capillary channels for shaping X-ray beams were first suggested by Arkdev and his collaborators in 1986 (Arkadev et al., 1989 [109]) The development and study of polycapillary optics and its applications in X-ray lithography (Klotzko et al., 1995 [110]), X-ray astronomy (Russell et al., 1997 [111], and Russell et al. 1999 [97]) diffraction analysis (Owens et al., 1997 [76], Kardiawarman et al. 1995 [112]), X-ray fluorescence (Gao et al., 1996 [113]) and medicine (Kruger et al., 1996 [114]) have been reported.

Like multichannel plates, they differ from single-bore capillaries in that the focusing or collecting effects come from the overlap of the beams from hundreds of thousands of channels, rather than from the action within a single tube (MacDonald and Gibson, 2010 [57]). X rays can be transmitted down a curved fibre as long as the fibre is 


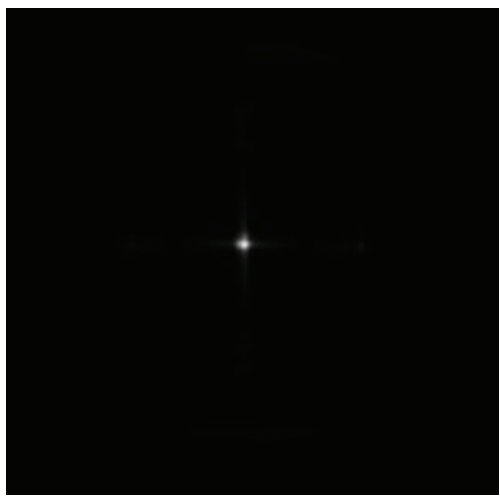

$280 \mathrm{eV}$

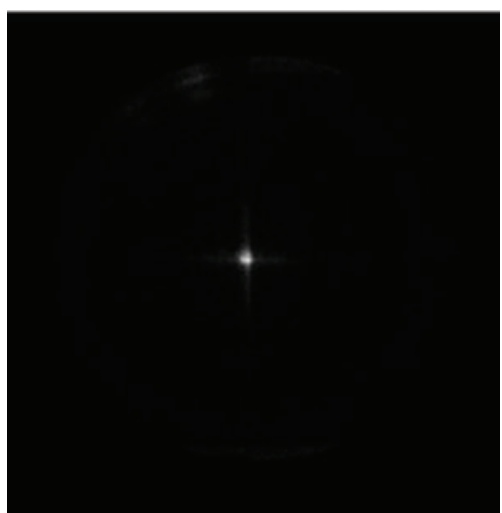

$2900 \mathrm{eV}$

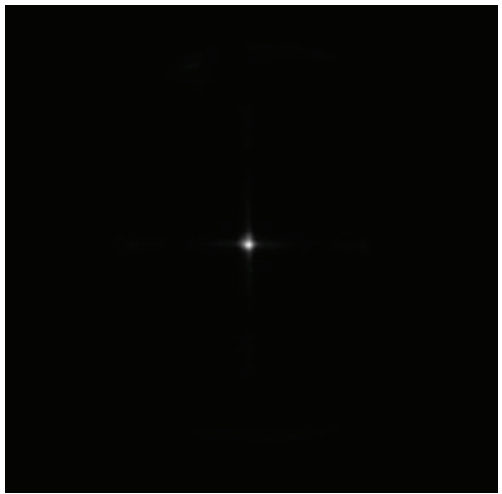

$930 \mathrm{eV}$

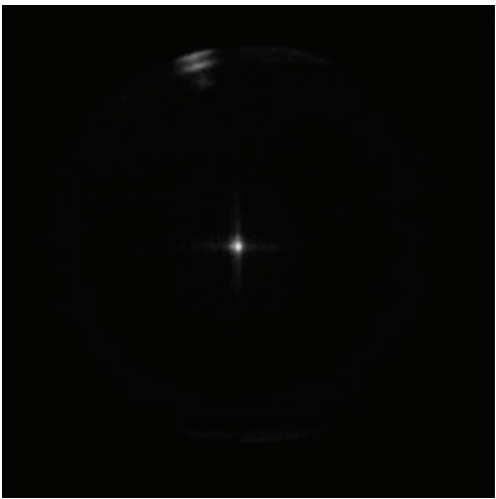

$4500 \mathrm{eV}$

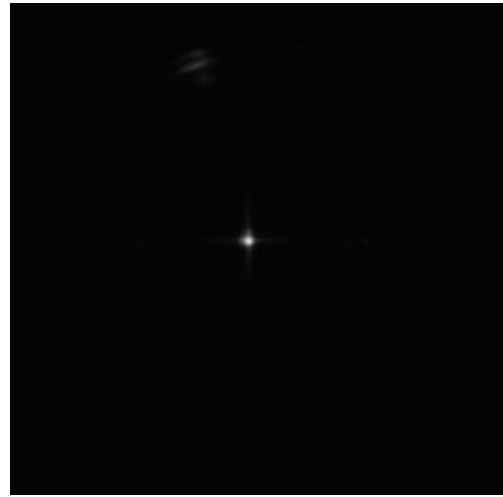

$1500 \mathrm{eV}$

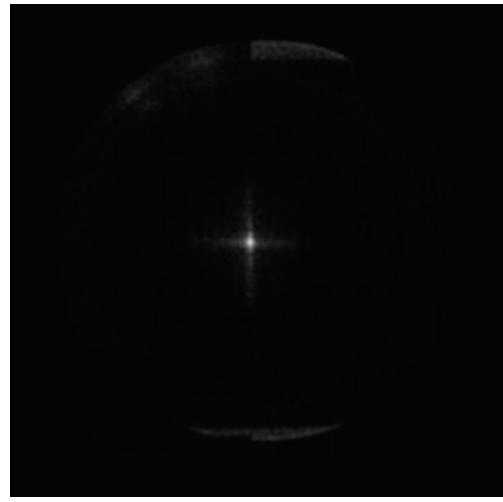

$8000 \mathrm{eV}$

Figure 55: X-ray images from MFO LE, $f=25 \mathrm{~cm}$, Palermo test facility (Tichy et al., 2010b [74]).

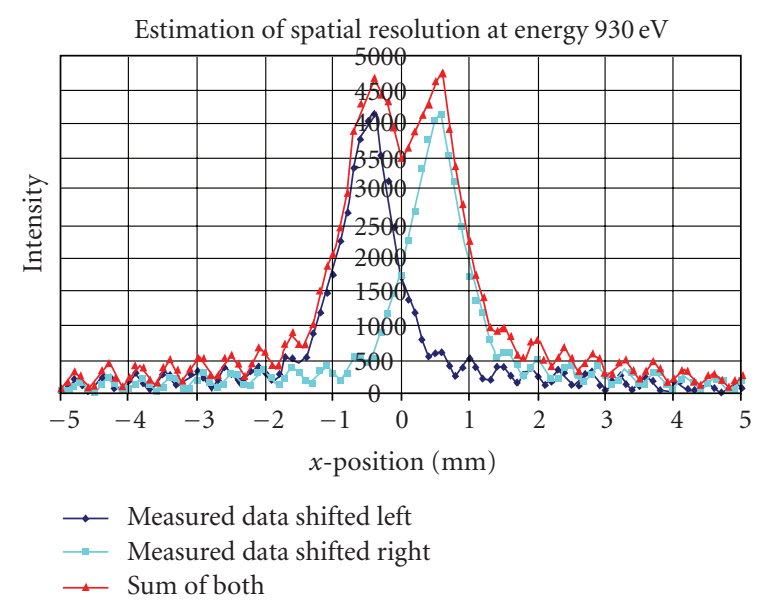

Figure 56: Measurements of MFO LE optics with $f=25 \mathrm{~cm}$ in the Palermo X-ray facility (measured FWHM of about 10 arcmin between 0.28 and $8 \mathrm{keV}$ (Tichy et al., 2010b [74]).

small enough and bent gently enough to keep the angles of incidence less than the critical angle. The angle of incidence for the ray near one edge increases with tube diameter. The requirement that the incident angles remain less than the critical angle necessitates the use of tiny tube diameters. However, mechanical limitations prohibit the manufacture of capillary tubes with outer diameters smaller than about 300 microns. For this reason, polycapillary fibres are employed. Typical channel sizes are between 2 and 12 microns. Thousands of such fibres are strung through lithographically produced metal grids to produce a multifibre lens. Alternatively, a larger-diameter polycapillary fibre can be shaped into a monolithic optic.

Polycapillary X-ray optics is a powerful, relatively new control technology for X-ray beams (MacDonald and Gibson, 2010 [57]). Using polycapillary optics to collimate the output from a point source provides in most cases much higher intensity than pinhole collimation, particularly if 2dimensional collimation is required. Collimating optics that collect from 0.7 to 3 millisteradian of a divergent beam has an output diameter range from 1 to $6 \mathrm{~mm}$ for a monolithic optic to $\geq 3 \mathrm{~cm}$ for a multifibre collimating optic. Output divergence varies inversely with the photon energy, and is around $3 \mathrm{mrad}$ at $8 \mathrm{keV}$. Focusing the beam yields even higher-intensity gains. Measured focused beam gains for sample sizes from 0.3 to $0.5 \mathrm{~mm}$ are about a factor of 100 , and agree well with computations (MacDonald and Gibson, 2010 [57], and references therein). Computed gains for smaller samples are even higher. Polycapillary optics can also be used to perform low pass spectral filtering, which allows the use of increased source voltage. Further, the optics also removes the connection between source size and resolution, which allows the use of increased source current. Increasing the voltage 

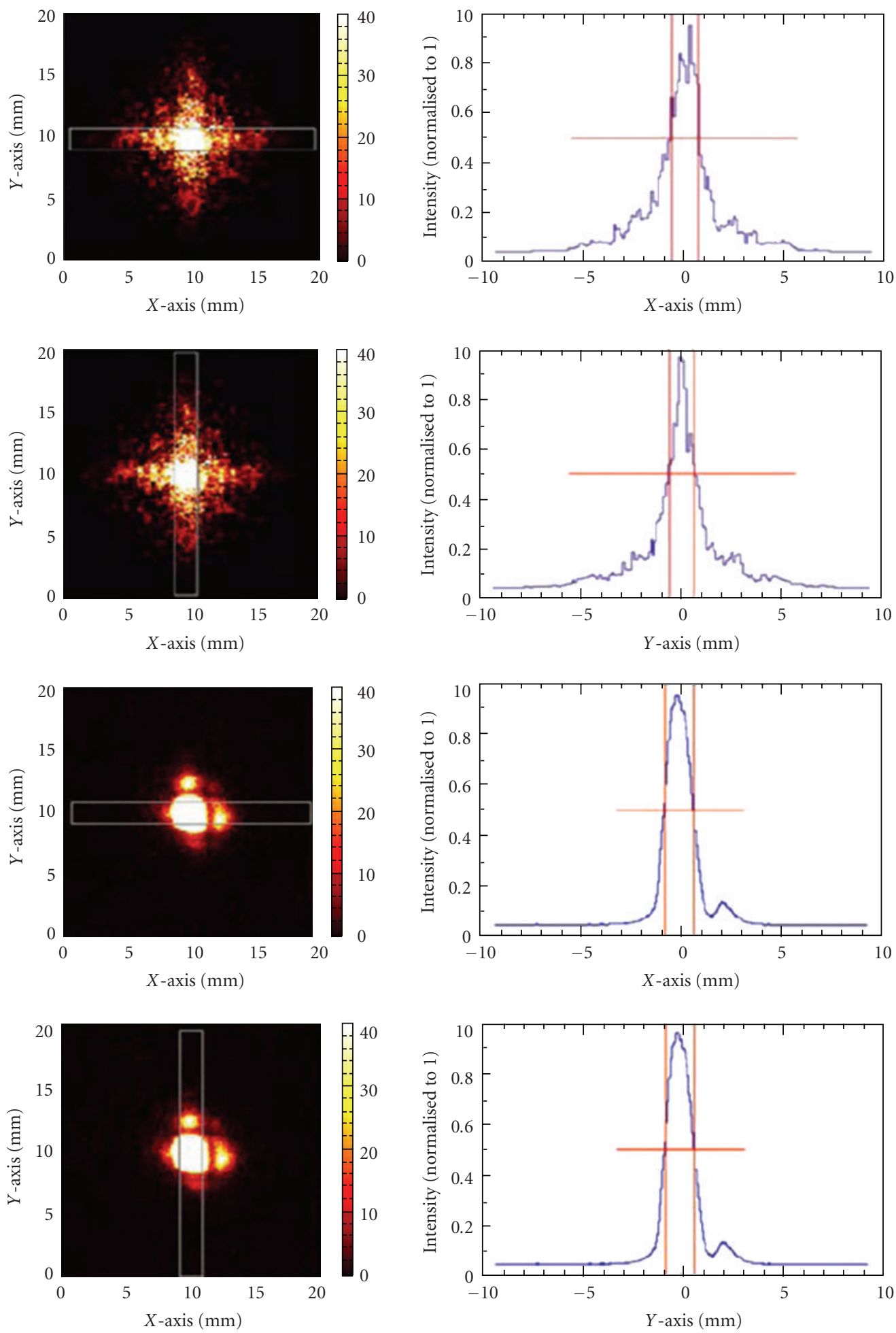

FIGURE 57: Upper panel: simulated LE MCP $f=3.8 \mathrm{~cm}$ optic performance at a wavelength of 121.6 misalignments have been modelled by introducing an additional tilt to each and direction of each tilt is taken from a Gaussian distribution with a FWHM X-axis and $1.70^{\circ}$ in the $y$-axis. The resulting focal spot has a FWHM (horizontal) and $2.64^{\circ} \pm 0.2^{\circ}$ (vertical). Lower panel: laboratory measurements. Cross-arm structures characteristic of a square-pore MCP optic are clearly visible. The FWHM of the central focus is 2.38 deg (horizontal) $\times 2.70$ deg (vertical) (Bannister et al., 2007 [75]). 
and current of the source increases the useful intensity. While not true imaging optics, polycapillary fibres can transmit an image in the same manner as a coherent fibre bundle. Polycapillary optics can be used to magnify and demagnify images and to remove the high-angle Compton scattering which can otherwise result in substantive image degradation.

\section{Summary}

The grazing incidence X-ray optical elements of nonWolter type, mainly Kirkpatrick-Baez and lobster-eye optical systems, offer alternative solution for many various future goals both in space and in laboratory. They can offer cheaper, and/or lighter alternatives as well as a larger field of view. At the same time, new computer-based systems allow to consider alternative and arrangements which were impossible before.

Some of these systems, such as KB optics, have already found wide applications in laboratory at synchrotron, demonstrated their performance and advantages. This includes also further modifications and improvements of these systems. It is obvious that for example, KB systems have fully demonstrated their superior imaging performance and accuracy.

The KB microscopes have found wide application in many laboratory areas. Different situation is in the field of telescope applications: here the KB telescopes were used in the past on sounding rockets in the 1970s, and discussed for satellite applications after, but eventually have never flown on a satellite. This situation can however change with introduction of new type of substrates, new and better alignment control. Furthermore, funding pressures may lead to serious consideration of $\mathrm{KB}$ optics as an alternative to Wolter-1 optics for large-area X-ray telescopes, owing to the lower cost of the former systems.

The drawback of KB systems if compared to Wolter 1 system that is, the longer focal length to achieve the same effective area and performance can be solved by some kind of novel advanced deployable techniques.

While the historical KB telescope applications were based on thin sheets of float glass, the recently available improved Silicon wafers represent an even more promising alternative, especially for segmented telescopes, as they have better optical surfaces and a very low microroughness (due to chemicalmechanical polishing). In our understanding, the early $\mathrm{KB}$ stacks based on $\mathrm{Si}$ wafers were unable to demonstrate the full capacity of $\mathrm{KB}$ imaging as the early substrates were of inadequate quality for X-ray optics applications. Si wafers with considerably improved parameters and flatness have been available recently. There are also novel methods how to improve further the quality of thin float glass (e.g., by thermal forming on precise flat mandrels).

The results obtained in the field of very wide field Xray telescopes of lobster-eye type seem to be promising very much. The prototypes developed and tested confirm that these telescopes are fully feasible and can achieve fine angular resolutions of $4 \mathrm{arcmin}$ or better over a wide field of view, as well as high gain to up to $3 \mathrm{keV}$ and still reasonable gain up to $10 \mathrm{keV}$ of the Schmidt arrangement and MFO technology is used. The modular concept allows using the detector techniques recently available and also allows modifying the telescope design for a dedicated experiment. The low weight of both optic as well as detector allows considering LE monitors to be flown on a small satellites, in extreme case even picosatellites. These new devices are ready for X-ray astronomy applications and are expected to help to solve various questions of recent X-ray astrophysics. In addition to that, there are also wide application possibilities in laboratory.

\section{Acknowledgments}

The works performed in the Czech Republic partly described here are based on a wide collaboration of several Czech Institutions, namely the teams from Czech Technical University, Faculty of Nuclear Science, Prague, Institute of Chemical Technology in Prague, RITE Rigaku Innovative Technologies Europe, Prague, ON Semiconductor Czech Republic, Astronomical Institute of the Academy of Sciences of the Czech Republic, Ondrejov, and many others. The investigations of lobster (crayfish eyes) have been supported recently by the involvement of Faculty of natural science of the Charles University in Prague, Dr. Adam Petrusek and Petr Jan Juracka. The development of X-ray optics in the Czech Republic is also supported by several grants and projects. The design and development of innovative X-ray telescopes was supported by the grants 106/97/1223 and 102/99/1546 provided by the Grant Agency of the Czech Republic. The authors also acknowledges the support from the Ministry of Industry and Trade of the Czech Republic, Projects nos. FB-C3/29/00 and FD-K3/052. More recently, the authors acknowledges the support provided by the Grant Agency of the Academy of Science of the Czech Republic, Grant nos. IAAX01220701, by the Ministry of Education and Youth of the Czech Republic, Projects nos. ME918 and ME09028 and by Ministry of Industry and Trade of the Czech Republic, FD-K3/052. The investigations related to the ESA IXO project are supported by the ESA PECS Project no. 98039. M.S. was supported by the junior grant by the Grant Agency of the Czech Republic, Grant no. 202/07/P510. The KB optics development is supported by the Ministry of Education and Youth of the Czech Republic, Project no. ME09004.

\section{References}

[1] H. Wolter, "Spiegelsysteme streifenden Einfalls als abbildende Optiken für Röntgenstrahlen," Annalen der Physik, vol. 10, pp. 94-114, 1952.

[2] P. Kirkpatrick and A. V. Baez, "Formation of optical images by X-rays," Journal of the Optical Society of America, vol. 38, no. 9, pp. 766-773, 1948.

[3] 2010, http://www.X-ray-optics.de/.

[4] M. A. Kumakhov, "Channeling of photons and new X-ray optics," Nuclear Inst. and Methods in Physics Research, B, vol. 48, no. 1-4, pp. 283-286, 1990. 
[5] J. W. Kast, "Scanning kirkpatrick-baez x-ray telescope to maximize effective area and eliminate spurious images; design," Applied Optics, vol. 14, no. 2, pp. 537-545, 1975.

[6] P. Gorenstein, B. Harris, H. Gursky, and R. Giacconi, "A rocket payload using focusing X-ray optics for the observations of soft cosmic X-rays," Nuclear Instruments and Methods, vol. 91, pp. 451-459, 1971.

[7] P. Gorenstein, E. Whitbeck, K. Gerald et al., "Lobster-eye Xray telescope prototype," in Multilayer and Grazing Incidence X-Ray/EUV Optics III, vol. 2805 of Proceedings of SPIE, p. 74, 1996.

[8] V. Marsikova, "X-ray optics: wolter," in Proceedings of the International Workshop on Astronomical X-Ray Optics, Prague, Czech Republic, December 2009.

[9] H. Sabet, K. Ishii, S. Matsuyama et al., "A method to modify coordinates of detectors in positron emission tomography systems," Nuclear Instruments and Methods in Physics Research A, vol. 600, no. 3, pp. 678-682, 2009.

[10] R. Kodama, N. Ikeda, Y. Kato, Y. Katori, T. Iwai, and K. Takeshi, "Development of an advanced Kirkpatrick-Baez microscope," Optics Letters, vol. 21, no. 17, pp. 1321-1323, 1996.

[11] R. Mokso, P. Cloetens, E. Maire, W. Ludwig, and J.-Y. Buffière, "Nanoscale zoom tomography with hard $\mathrm{X}$ rays using Kirkpatrick-Baez optics," Applied Physics Letters, vol. 90, no. 14, Article ID 144104, 3 pages, 2007.

[12] S. J. Chen, S. Y. Perng, P. C. Tseng, C. K. Kuan, D. J. Wang, and C. T. Chen, "K-B microfocusing system using monolithic flexure-hinge mirrors for synchrotron X-rays," Nuclear Instruments and Methods in Physics Research A, vol. 467-468, pp. 283-286, 2001.

[13] A. Ivan, R. J. Bruni, S. B. Cenko, P. Gorenstein, and S. E. Romaine, "High-energy characterization of multilayers for hard X-ray astronomy," in X-Ray Optics for Astronomy: Telescopes, Multilayers, Spectrometers, and Missions, vol. 4496 of Proceedings of SPIE, pp. 134-139, July 2001.

[14] K. D. Joensen, P. Gorenstein, J. L. Wood, F. E. Christensen, and P. Hoghoj, "Preliminary results of a feasibility study for a hard X-ray Kirkpatrick-Baez telescope," in Advances in Multilayer and Grazing Incidence X-Ray/EUV/FUV Optics, vol. 2297 of Proceedings of SPIE, pp. 180-189, July 1994.

[15] L. Sveda, Astrophysical aspects of lobster eye X-ray telescopes, Ph.D. thesis, Charles University Prague, Faculty of Mathematics and Physics, Prague, Czech Republic, 2003.

[16] M. von Laue, "Eine quantitative Prüfung der Theorie für die Interferenzerscheinungen bei Röntgenstrahle," Proceedings of the Bavarian Academy of Sciences, vol. 72, no. 5, pp. 303-322, 1912.

[17] H. Compton, "The spectrum of scattered X-rays," Physical Review, vol. 22, no. 5, pp. 409-413, 1923.

[18] W. Ehrenberg and F. Jentzsch, "Über die Auslösung von photoelektronen durch röntgenstrahlen aus metallspiegeln an der grenze der totalreflexion," Zeitschrift für Physik, vol. 54, no. 3-4, pp. 227-235, 1929.

[19] F. Jentzch, "Optische versuche mit rontgenstrahlen," Zeitschrift für Physik, vol. 30, p. 1268, 1929.

[20] T. R. Burnight, "Soft X-radiation in the upper atmosphere," Physical Review, vol. 76, p. 165, 1949.

[21] T. A. Chubb, H. Friedman, R. W. Kreplin, R. L. Blake, and A. E. Unzicker, Mémoires de la Société Royale des Sciences de Liège, vol. 4, p. 235, 1961.
[22] E. T. Byram, T. A. Chubb, and H. Friedman, "Ultraviolet light from celestial sources," Universite de Liege, Institute of Astrophysics College, vol. 8, no. 418, pp. 469-475, 1961.

[23] R. Hudec, B. Valnicek, V. Hudcova, J. Sylwester, and Z. Kordylewski, "-ray pictures of the sun taken from Vertical 8," Astronomical Institutes of Czechoslovakia, Bulletin, vol. 35, no. 3, pp. 153-158, 1984.

[24] J. H. Underwood, J. E. Milligan, A. C. deLoach, and R. B. Hoover, "S056 X-ray telescope experiment on the Skylab Apollo Telescope Mount," Applied Optics, vol. 16, no. 4, pp. 858-869, 1977.

[25] J. H. Underwood and D. T. Attwood, "The renaissance of $x-$ ray optics," Physics Today, vol. 34, p. 44, 1984.

[26] D. J. Thiel, E. A. Stern, D. H. Bilderback, and A. Lewis, "Focusing of synchrotron radiation using tapered glass capillaries," Physica B, vol. 158, no. 1-3, pp. 314-316, 1989.

[27] J. Underwood and T. W. Barbee, "Layered synthetic microstructures as Bragg diffractors for X rays and extreme ultraviolet: theory and predicted performance," Applied Optics, vol. 20, no. 17, pp. 3027-3034, 1981.

[28] R. Giacconi and B. Rossi, "A 'telescope' for soft X-ray astronomy," Journal of Geophysical Research, vol. 65, p. 773, 1960.

[29] J. D. Mangus and J. H. Underwood, "Optical design of a glancing incidence X-ray telescope," Applied Optics, vol. 76, no. 6, pp. 95-102, 1969.

[30] R. Hudec, B. Valnicek, B. Aschenbach, H. Brauninger, and W. Burkert, "Grazing incidence replica optics for astronomical and laboratory applications," Applied Optics, vol. 27, no. 8, pp. 1453-1455, 1988.

[31] O. Citterio, M. Ghigo, F. Mazzoleni et al., "Development of soft and hard X-ray optics for astronomy: progress report II and considerations on material properties for large diameter segmented optics of future missions," in X-Ray Optics for Astronomy: Telescopes, Multilayers, Spectrometers, and Missions, vol. 4496 of Proceedings of SPIE, pp. 23-40, July 2001.

[32] R. Hudec, L. Pina, A. Inneman et al., "Innovative technologies for future astronomical X-ray mirrors," in UV and Gamma-Ray Space Telescope Systems, vol. 5488 of Proceedings of SPIE, pp. 875-885, June 2004.

[33] R. Hudec, L. Pina, A. Inneman et al., "Novel technologies for X-ray multi-foil optics," in Optics for EUV, X-Ray, and Gamma-Ray Astronomy II, O. Citterio and S. L. O'Dell, Eds., vol. 5900 of Proceedings of the SPIE, pp. 276-287, 2005.

[34] R. Hudec, A. V. Inneman, L. Pina, V. Hudcova, H. Sveda, and L. Ticha, "Lobster-eye X-ray telescopes: recent progress," in X-Ray and Gamma-Ray Telescopes and Instruments for Astronomy, J. E. Truemper and H. D. Tananbaum, Eds., vol. 4851 of Proceedings of the SPIE, pp. 578-586, 2003.

[35] I. Sobelman, I. A. Zhitnik, B. Valnicek et al., "Images of the sun obtained with the TEREK X-ray telescope on Phobos1," Pis'ma v Astronomicheskii Zhurnal, vol. 16, pp. 323-329, 1990.

[36] R. Hudec, B. Valnicek, V. Prazak, and I. Solc, "Properties of galvanoplastic grazing incidence mirrors for astronomical applications," Astronomical Institutes of Czechoslovakia, Bulletin, vol. 31, no. 3, pp. 188-191, 1981.

[37] R. Hudec and B. Valníček, "Development of X-ray mirrors for high-energy astrophysics in czechoslovakia," Advances in Space Research, vol. 3, no. 10-12, pp. 545-547, 1984.

[38] S. K. Sinha, E. B. Sirota, S. Garoff, and H. B. Stanley, "X-ray and neutron scattering from rough surfaces," Physical Review B, vol. 38, no. 4, pp. 2297-2311, 1988. 
[39] R. Hudec, B. Valnicek, L. Svatek, and V. Landa, "Development and production of ultrathin X-ray mirrors," in EUV, $X$-Ray, and Gamma-Ray Instrumentation for Astronomy and Atomic Physics, Proceedings of SPIE, pp. 617-624, Society of Photo-ptical Instrumentation Engineers, San Diego, Calif, USA, August 1989.

[40] R. Hudec, B. Valnicek, L. Svatek, and V. Landa, "New developments in replica X-ray grazing incidence optics," in X-Ray Instrumentation in Medicine and Biology, Plasma Physics, Astrophysics, and Synchrotron Radiation, R. Benattar, Ed., vol. 1140 of Proceedings of SPIE, 1989.

[41] R. Hudec, B. Valnicek, J. Cervencl et al., "Recent developments in production of thin X-ray reflecting foils," in $X$ Ray/EUV Optics for Astronomy, Microscopy, Polarimetry, and Projection Lithography, vol. 1343 of Proceedings of SPIE, pp. 162-163, July 1990.

[42] R. Hudec, A. Inneman, U. Arndt, and L. Pina, "Compact laboratory X-ray Beam Line with focusing microoptics," in Proceedings of the International Congress of X-Ray Optics and Microanalysis, Institute of Physics Conference Series, chapter 7, Manchester, UK, 1992.

[43] R. Hudec, A. Inneman, U. W. Arndt, and L. Pina, "Replicated X-ray micromirrors," in Proceedings of the l3th International Conference on X-Ray Opticsand Microanalysis, IOP Conference Series, Manchester, UK, 1992.

[44] R. Hudec, L. Pina, and A. Inneman, "Replicated grazing incidence X-ray optics: past, present, and future," in X-Ray Optics, Instruments, and Missions II, vol. 3766 of Proceedings of SPIE, p. 62, July 1999.

[45] R. Hudec, "Innovative technologies for future X-ray telescopes," in Proceedings of the Astronomical Society of the Pacific, H. Inoue and H. Kunieda, Eds., vol. 251 of New Century of X-ray Astronomy, p. 44, Astronomical Society of the Pacific, San Francisco, Calif, USA, 2001.

[46] R. Hudec, "Wide-field sky monitoring — optical and X-rays," Memorie della Societa Astronomic Ataliana, vol. 74, p. 973, 2003.

[47] R. Hudec, L. Sveda, A. Inneman, and L. Pina, "Astronomical lobster eye telescopes," in UV and Gamma-Ray Space Telescope Systems, vol. 5488 of Proceedings of SPIE, pp. 449-459, June 2004.

[48] B. L. Henke, E. M. Gullikson, and J. C. Davis, "X-ray interactions: photoabsorption, scattering, transmission, and reflection at $\mathrm{E}=50-30,000 \mathrm{eV}, \mathrm{Z}=1-92$," Atomic Data and Nuclear Data Tables, vol. 54, no. 2, pp. 181-342, 1993.

[49] J. H. Hubbell, W. M. J. Veigele, E. A. Briggs, R. T. Brown, D. T. Cromer, and R. J. Howerton, "Atomic form factors, incoherent scattering functions, and photon scattering cross sections," Journal of Physical and Chemical Reference Data, vol. 4, no. 3, pp. 471-538, 1975.

[50] M. Kopecký, "Diffuse scattering of x rays from nonideal layered structures," Journal of Applied Physics, vol. 77, no. 6, pp. 2380-2387, 1995.

[51] A. H. Compton, "Incoherent scattering and the concept of discrete electrons," Physical Review, vol. 47, no. 5, pp. 367370, 1935.

[52] B. Aschenbach, "X-ray telescopes," Reports on Progress in Physics, vol. 48, no. 5, pp. 579-629, 1985.

[53] W. K. H. Schmidt, "A proposed X-ray focusing device with wide field of view for use in X-ray astronomy," Nuclear Instruments and Methods, vol. 127, no. 2, pp. 285-292, 1975.
[54] M. Bavdaz, D. H. Lumb, A. Peacock, M. Beijersbergen, and S. Kraft, "Development of X-ray optics for the XEUS mission," in X-Ray Sources and Optics, C. A. MacDonald, A. T. Macrander, T. Ishikawa, C. Morawe, and J. L. Wood, Eds., vol. 5539 of Proceedings of the SPIE, pp. 95-103, 2004.

[55] A. Bartnik, H. Fiedorowicz, R. Jarocki et al., "Lobster eye optics for collecting radiation of a laser-plasma soft X-ray source based on a gas puff target," Advances in X-Ray Analysis, vol. 49, p. 395, 2006.

[56] L. Sveda, V. Semencova, A. Inneman, L. Pina, and R. Hudec, "Hybrid lobster optic", in RenePublication: Laser-Generated, Synchrotron, and Other Laboratory X-Ray and EUV Sources, Optics, and Applications II, G. A. Kyrala, J.-C. J. Gauthier, C. A. MacDonald, and A. M. Khounsary, Eds., vol. 5918 of Proceedings of SPIE, pp. 22-30, 2005.

[57] C. A. MacDonald and W. M. Gibson, "Polycapillary and multichannel plate X-ray optics," in Handbook of Optics, vol. 5 of Atmospheric Optics, Modulators, Fiber Optics, X-Ray and Neutron Optics, 3rd edition, 2010.

[58] M. Montel, X-Ray Microscopy with Catamegonic Roof Mirrors, X-Ray Microscopy and Microradiography, Academic Press, New York, NY, USA, 1957.

[59] C. S. Alford, R. M. Bionta, D. M. Makowiecki et al., "Molybdenum/beryllium multilayer mirrors for normal incidence in the extreme ultraviolet," Applied Optics, vol. 34, no. 19, pp. 3727-3730, 1995.

[60] J. R. P. Angel, “Lobster eyes X-ray telescopes," The Astrophysical Journal, vol. 233, p. 364, 1979.

[61] P. Gorenstein, "All sky supernova and transient explorer (ASTRE)," in Variability of Galactic and Extragalactic XRay Sources, A. Treves, Ed., Associazione per L'Avanzamento dell'Astronomia, Milano, Italy, 1987.

[62] W. C. Priedhorsky, A. G. Peele, and K. A. Nugent, "An Xray all-sky monitor with extraordinary sensitivity," Monthly Notices of the Royal Astronomical Society, vol. 279, no. 3, pp. 733-750, 1996.

[63] G. Fraser, 2002, http://www.src.le.ac.uk/lobster.

[64] G. W. Fraser, "An imaging All-sky monitor (ASM) based on Lobster Eye X-ray optics. An international space station (ISS) attached payload submitted in response to the ESA call for mission proposals for the flexi-missions F2 and F3," Bulletin of the American Astronomical Society, vol. 35, p. 865, 2003.

[65] G. W. Fraser, A. N. Brunton, N. P. Bannister et al., "LobsterISS: an imaging X-ray all-sky monitor for the International Space Station," in X-Ray and Gamma-Ray Instrumentation for Astronomy XII, Proceedings of SPIE, pp. 115-126, August 2001.

[66] G. W. Fraser, J. D. Carpenter, D. A. Rothery et al., "The mercury imaging X-ray spectrometer (MIXS) on bepicolombo," Planetary and Space Science, vol. 58, no. 1-2, pp. 79-95, 2010.

[67] A. Inneman, R. Hudec, L. Pina, and P. Gorenstein, "Lobstereye X-ray optics," in X-Ray Optics, Instruments, and Missions II, vol. 3766 of Proceedings of SPIE, pp. 72-79, July 1999.

[68] A. Inneman, R. Hudec, and L. Pina, "Progress in Lobster-eye $\mathrm{X}$-ray optics development," in X-Ray Optics, Instrumants, and Missions IV, vol. 4138 of Proceedings of SPIE, pp. 94-104, July 2000.

[69] R. Hudec, B. Valnicek, I. Solc et al., "Wolter 1 type X-ray mirror system with mean resolution and maximum effective collecting area for the spectral region of 1-10 nm, In its Publ. of the Astron," Institute of Mathematics of the Academy of Sciences of the Czech, no. 61, pp. 1-4, 1985. 
[70] R. Hudec, L. Pina, A. V. Inneman et al., "Lightweight X-ray optics for future space missions," in X-Ray and Gamma-Ray Telescopes and Instruments for Astronomy, J. E. Truemper and H. D. Tananbaum, Eds., vol. 4851 of Proceedings of the SPIE, pp. 656-665, 2003.

[71] G. W. Fraser, A. N. Brunton, J. E. Lees et al., "Development of micro channel plate MCP X-ray optics," in Multilayer and Grazing Incidence X-Ray/EUV Optics III, vol. 2011 of Proceedings of SPIE, pp. 215-226, 1993.

[72] A. N. Brunton, G. W. Fraser, J. E. Lees, W. B. Feller, and P. L. White, "X-ray focusing with $11 \mu \mathrm{m}$ square porte microchannel plates," in X-Ray and Ultraviolet Sensors and Applications, vol. 2519 of Proceedings of the SPIE, 1995.

[73] V. Tichý, L. Švéda, J. Maršík et al., "Tests of imaging with lobster-eye X-ray optics and medipix2 detector," Baltic Astronomy, vol. 18, no. 3-4, pp. 369-373, 2009.

[74] V. Tichý, M. Hromćík, R. Hudec et al., "Tests of lobster-eye optics for a small X-ray telescope," Baltic Astronomy, vol. 18, no. 3-4, pp. 362-368, 2009.

[75] N. P. Bannister, E. J. Bunce, S. W. H. Cowley et al., "A wide field auroral imager (WFAI) for low earth orbit missions," Annales Geophysicae, vol. 25, no. 2, pp. 519-532, 2007.

[76] S. M. Owens, F. A. Hoffman, C. A. MacDonald, and W. M. Gibson, "Microdiffraction using collimating and convergent beam polycapillary optics," in Proceedings of the 46th Annual Denver X-Ray Conference, vol. 41 of Advances in X-Ray Analysis, pp. 314-318, Steamboat Springs, Colo, USA, August 1997.

[77] K. Wallace, M. Bavdaz, M. Collon et al., "X-ray pore optic developments," Reports on Progress in Physics, vol. 48, pp. 579-629, 1985.

[78] G. S. Vaiana, L. Van Speybroeck, M. V. Zombeck, A. S. Krieger, J. K. Silk, and A. Timothy, "The S-054 X-ray telescope experiment on SKYLAB," Space Science Instrumentation, vol. 3, no. 1, pp. 19-76, 1977.

[79] B. Aschenbach, "Realization of X-ray telescopes-from design to performance," Experimental Astronomy, vol. 26, no. 1-3, pp. 95-109, 2009.

[80] W. Ehrenberg, "X-ray optics," Nature, vol. 160, no. 4062, pp. 330-331, 1947.

[81] P. C. Fisher and A. J. Meyerott, "High sensitivity detectors for few-kev x-rays," IEEE Transactions on Nuclear Science, vol. NS-13, p. 580, 1966.

[82] P. Gorenstein, B. Harris, H. Gursky, R. Giacconi, R. Novick, and P. Vanden Bout, "X-ray structure of the Cygnus Loop," Science, vol. 172, no. 3981, pp. 369-372, 1971.

[83] L. P. Van Speybroeck, R. C. Chase, and T. F. Zehnpfennig, "Orthogonal mirror telescopes for X-ray astronomy," Applied Optics, vol. 10, p. 537, 1971.

[84] L. P. Van Speybroeck, R. C. Chase, and T. F. Zehnpfennig, "Orthogonal mirror telescopes for X-ray astronomy," Applied Optics, vol. 10, no. 4, pp. 945-949, 1971.

[85] R. Willingale and F. H. P. spaan, "The design, manufacture and predicted performance of kirkpatrick-baez silicon stacks for the international X-ray observatory or similar applications," in Optics for EUV, X-Ray, and Gamma-Ray Astronomy $I V$, vol. 7437 of Proceedings of SPIE, August 2009.

[86] P. Gorenstein, A. DeCaprio, R. Chase, and B. Harris, "Large area focusing collector for the observation of cosmic X rays," Review of Scientific Instruments, vol. 44, no. 5, pp. 539-545, 1973.
[87] M. C. Weisskopf, "Design of grazing-incidence X-ray telescopes. 1," Applied Optics, vol. 12, no. 7, pp. 1436-1439, 1973.

[88] R. C. Catura, P. C. Fisher, H. M. Johnson, and A. J. Meyerott, "Asymmetry of soft x-ray emission near M87," The Astrophysical Journal, vol. 177, p. L1, 1972.

[89] R. Borken, R. Doxsey, and S. Rappaport, "A soft x-ray survey of the galactic plane from Cygnus to norma," The Astrophysical Journal, vol. 178, p. L115, 1972.

[90] L. M. Cohen, "Optimum shape of a Kirkpatrick-Baez Xray reflector supported at discrete points for on-axis performance," Applied Optics, vol. 20, no. 9, pp. 1545-1549, 1981.

[91] P. Gorenstein, H. Gursky, F. R. Harnden Jr., A. Decaprio, and P. Bjorkholm, "Large area soft X-ray imaging system for cosmic X-ray studies from rockets," IEEE Transaction on Nuclear Science, vol. NS-22, pp. 616-619, 1975.

[92] P. Gorenstein, "Deployable ultrahigh-throughput X-ray telescope: concept ," in X-Ray Optics, Instruments, and Missions, vol. 3444 of Proceedings of SPIE, p. 382, 1998.

[93] M. K. Joy, J. J. Kolodziejczak, M. C. Weisskopf, S. B. Fair, and B. D. Ramsey, "Imaging properties of a silicon wafer X-ray telescope," in Advances in Multilayer and Grazing Incidence X-Ray/EUV/FUV Optics, vol. 2297 of Proceedings of SPIE, pp. 283-286, July 1994.

[94] J. F. McGee, "A long-wavelength X-ray reflection microscope," in X-Ray Microscopy and Microradiography, V. E. Cosslett, A. Engstrom, and H. H. Patte, Eds., p. 164, Academic Press, New York, NY, USA, 1957.

[95] A. Iida and K. Hirano, "Kirkpatrick-Baez optics for a sub$\mu \mathrm{m}$ synchrotron X-ray microbeam and its applications to X-ray analysis," Nuclear Instruments and Methods in Physics Research B, vol. 114, no. 1-2, pp. 149-153, 1996.

[96] G. E. Ice, C. R. Hubbard, B. C. Larson et al., "KirkpatrickBaez microfocusing optics for thermal neutrons," Nuclear Instruments and Methods in Physics Research A, vol. 539, no. 1-2, pp. 312-320, 2005.

[97] H. Russell, M. Gubarev, J. Kolodziejczak, M. Joy, C. A. MacDonald, and W. M. Gibson, "Polycapillary X-ray optics for X-ray astronomy," in Proceedings of the 48th Denver X-Ray Conference, vol. 43 of Advances in X-Ray Analysis, 1999.

[98] A. Inneman, Technological aspects of the development and construction of optical elements for X-rays, Ph.D. thesis, Czech Technical University in Prague, Faculty of Mechanical Engineering, Prague, Czech Republic, 2001.

[99] P. Gorenstein, "X-ray optics for the LAMAR facility, an overview," Proceedings of the Society of Photo-Optical Instrumentation Engineers, vol. 184, pp. 63-72, 1979.

[100] A. Inneman, L. Pina, and R. Hudec, "Development of foil segments for large astronomical X-ray telescopes," in X-Ray Optics for Astronomy: Telescopes, Multilayers, Spectrometers, and Missions, P. Gorenstein and R. B. Hoover, Eds., vol. 4496 of Proceedings of SPIE, 2002.

[101] A. Inneman, L. Pina, R. Hudec, H. Ticha, and V. Brozek, "Innovative X-ray optics for astrophysics," in Design and Microfabrication of Novel X-Ray Optic, D. C. Mancini, Ed., vol. 4783 of Proceedings of SPIE, pp. 156-164, 2002.

[102] H. N. Chapman, K. A. Nugent, and S. W. Wilkins, "X-ray focusing using square channel-capillary arrays," Review of Scientific Instruments, vol. 62, no. 6, pp. 1542-1561, 1991.

[103] A. G. Peele, "Deconvolution of an extended object viewed by a lobster-eye telescope," Nuclear Instruments and Methods in Physics Research A, vol. 459, no. 1-2, pp. 354-364, 2001. 
[104] E. A. Stern, Z. Kalman, A. Lewis, and K. Lieberman, "Simple method for focusing $\mathrm{x}$ rays using tapered capillaries," Applied Optics, vol. 27, pp. 5135-5139, 1988.

[105] P. Engström, S. Larsson, A. Rindby et al., "A submicron synchrotron X-ray beam generated by capillary optics," Nuclear Instruments and Methods A, vol. 302, no. 3, pp. 547$552,1991$.

[106] D. X. Balaic and K. A. Nugent, "X-ray optics of tapered capillaries," Applied Optics, vol. 34, no. 31, pp. 7263-7272, 1995.

[107] U. W. Arndt, P. Duncumb, J. V. P. Long, L. Pina, and A. Inneman, "Focusing mirrors for use with microfocus X-ray tubes," Journal of Applied Crystallography, vol. 31, no. 5, pp. 733-741, 1998.

[108] L. Pina, A. Inneman, and R. Hudec, "Optics for X-ray and laser plasma soft X-ray radiation," in High Power LasersScience and Engineering, R. Kossowsky, M. Jelinek, and R. F. Walter, Eds., NATO ASI Series, pp. 373-380, Kluwer Academic, Dodrecht, The Netherlands, 1996.

[109] V. A. Arkd'ev, A. I. Kolomitsev, M. A. Kumakhov et al., "Wide-band X-ray optics with a large angular aperture," Soviet Physics-Uspekhi, vol. 32, no. 3, p. 271, 1989.

[110] Q. Klotzko, Q. F. Xiao, D. M. Gibson et al., "Investigation of glass polycapillaries for use in proximity X-ray lithography," in Applications of Laser Plasma Radiation II, vol. 2523 of Proceedings of SPIE, pp. 175-182, 1995.

[111] H. Russell, W. M. Gibson, M. V. Gubarev et al., "Application of polycapillary optics for hard X-ray astronomy," in Grazing Incidence and Multilayer X-Ray Optical Systems, R. B. Hoover and A. B. C. Walker II, Eds., vol. 3113 of Proceedings of SPIE, pp. 369-377, 1997.

[112] R. Kardiawarman, R. York, X.-W. Qian, Q.-F. Xiao, C. A. MacDonald, and W. M. Gibson, "Application of a multifiber collimating lens to thin film structure analysis," in X-Ray and Ultraviolet Sensors and Applications, R. B. Hoover and M. B. Williams, Eds., vol. 2519 of Proceedings of SPIE, pp. 197-206, July 1995.

[113] N. Gao et al., "Monolithic polycapillary focussing optics and their applications in microbeam X-ray fluorescence," Applied Physics Letters, vol. 69, no. 11, pp. 1529-1531, 1996.

[114] D. G. Kruger, C. C. Abreu, E. G. Hendee et al., "Imaging characteristics of X-ray capillary optics in digital mammography," Medical Physics, vol. 23, no. 2, pp. 187-196, 1996. 